\title{
POLYCYCLIC CODES OVER GALOIS RINGS WITH APPLICATIONS TO REPEATED-ROOT CONSTACYCLIC CODES
}

\author{
SERGIO R. LÓPEZ-PERMOUTH ${ }^{1}$, HAKAN ÖZADAM ${ }^{2}$, FERRUH ÖZBUDAK ${ }^{2}$, STEVE SZABO ${ }^{1}$ \\ ${ }^{1}$ Department of Mathematics \\ Ohio University, Athens, Ohio, 45701, USA \\ \{lopez,szabo\}@math.ohiou.edu \\ ${ }^{2}$ Department of Mathematics and Institute of Applied Mathematics \\ Middle East Technical University, İnönü Bulvarı, 06531, Ankara, Turkey \\ \{ozhakan,ozbudak\}@metu.edu.tr
}

\begin{abstract}
Cyclic, negacyclic and constacyclic codes are part of a larger class of codes called polycyclic codes; namely, those codes which can be viewed as ideals of a factor ring of a polynomial ring. The structure of the ambient ring of polycyclic codes over $G R\left(p^{a}, m\right)$ and generating sets for its ideals are considered. It is shown that these generating sets are strong Groebner bases. A method for finding such sets in the case that $a=2$ is also given. The Hamming distance of certain constacyclic codes of length $\eta p^{s}$ and $2 \eta p^{s}$ over $\mathbb{F}_{p^{m}}$ is computed. A method, which determines the Hamming distance of the constacyclic codes of length $\eta p^{s}$ and $2 \eta p^{s}$ over $G R\left(p^{a}, m\right)$, where $(\eta, p)=1$, is described. In particular, the Hamming distance of all cyclic codes of length $p^{s}$ over $G R\left(p^{2}, m\right)$ and all negacyclic codes of length $2 p^{s}$ over $\mathbb{F}_{p^{m}}$ is determined explicitly.
\end{abstract}

Keywords: Linear codes, cyclic codes, constacyclic codes, Galois rings, Groebner basis, repeated-root cyclic codes, torsion codes

\section{INTRODUCTION}

Important applications of modules over finite rings to error-correcting codes and sequences were introduced in [17] and [21. In particular, [17] motivated the study of cyclic and negacyclic codes over Galois rings (see, for example, 11, 5, 4, 14, 19, 35, 30, 36, 37]). For a recent survey on this topic, we refer the reader to [13]. Cyclic codes can be grouped into two classes: simple-root cyclic codes, where the codeword length and the characteristic of the alphabet are coprime, and repeated-root cyclic codes, where the codeword length and the characteristic of the alphabet are not coprime. The structure of simple-root cyclic codes over rings was studied throughly in [30, 19, 6, 14] and certain special generating sets for these codes were determined therein. On the other hand, repeated-root cyclic codes are also interesting as they allow very simple syndrome-forming and decoding circuitry and because in some cases (see [23, 31]) they are maximum distance separable. A partial list of references for the theory of repeated root cyclic codes includes [7, 8, 9, 10, 11, 12, 15, 22, 23, 31, 20, 32, 33, 34, 38. Amongst these studies, generating sets that are similar to those in [30, 19, 6, 14, are studied in [22, 15, 20, for cyclic codes of length $p^{s}$ over an alphabet whose characteristic is a power $p$. In [15, 20, the notion of torsional codes is used to study generators of these codes. The structural properties of cyclic codes are studied in a 
more general setting in [27, 26, 28, 25, 32] from a Groebner basis perspective. Our study unifies the two approaches above and generalizes them in the following sense: we show that codes in a wider class of linear codes called polycyclic codes have generating sets sharing the same properties as those described in $[27,26,28,25,32,15,20$. This allows us to study the ideal structure of cyclic codes without the restriction that the codes must be simple-root. In particular, we compute the Hamming distance of certain constacyclic codes of length $\eta p^{s}$ and $2 \eta p^{s}$, where $(\eta, p)=1$, over a finite field of characteristic $p$. Then using this result together with the above generating sets, we give a method to determine the Hamming distance of certain constacyclic codes of length $p^{s}$ and $2 p^{s}$ over a Galois ring of characteristic a power of $p$. As another particular case, we explicitly determine the Hamming distance of all cyclic codes of length $p^{s}$ over $G R\left(p^{2}, m\right)$ which generalizes the results of a recent study [18].

We study linear codes over Galois rings that have the additional structure that they can be described as an ideal of a quotient ring, specifically a quotient ring of a polynomial ring over a Galois ring where the ideal being factored out is generated by a regular polynomial. We begin with studying the structure of the ring $\frac{G R\left(p^{a}, m\right)[x]}{\langle g(x)\rangle}$ where $g(x)$ is a regular primary polynomial. We show that $\frac{G R\left(p^{a}, m\right)[x]}{\langle g(x)\rangle}$ is a local ring with a simple socle and we determine its maximal ideal and socle. We give necessary and sufficient conditions for $\frac{G R\left(p^{a}, m\right)[x]}{\langle g(x)\rangle}$ to be a chain ring. Next, we use the results on these rings to study the structure of $\frac{G R\left(p^{a}, m\right)[x]}{\langle f(x)\rangle}$ where $f(x)$ is a regular polynomial. This work uses a factorization given by [24] of regular polynomials into regular primary polynomials and also the Chinese Remainder Theorem. Via this ring decomposition, we give details on the structure of $\frac{G R\left(p^{a}, m\right)[x]}{\langle f(x)\rangle}$. This provides information on the structure of the polycyclic codes, and in particular cyclic and constacyclic codes, as their ambient spaces are of the form of $\frac{G R\left(p^{a}, m\right)[x]}{\langle f(x)\rangle}$. as their ambient spaces are of the form of $\frac{G R\left(p^{a}, m\right)[x]}{\langle f(x)\rangle}$.

Some special generating sets, for cyclic codes of length $p^{s}$ over $G R\left(p^{a}, m\right)$, were studied in [15] by employing torsional degrees and torsional codes. Later, in [20, Kiah et. al. came up with a unique set of generators for such codes. We generalize their results to polycyclic codes. More explicitly, we extend the notion of torsional degree and torsional code to polycyclic codes and we show that polycyclic codes have generating sets with the same properties as in [15] and [20]. Furthermore, we observe that the unique generating set studied in [20] is actually a strong Groebner basis which is studied in a series of papers [25, 26, 27, 28, 32] by Sălăgean and Norton. We show that a minimal strong Groebner basis actually gives us all the torsional degrees of a polycyclic code. This allows us to describe how to obtain a generating set in standard form, which is a minimal strong Groebner basis, from the unique generating set introduced in [20] and vice versa. Also the torsional degrees, equivalently a minimal strong Groebner basis, can be used to determine the Hamming distance of a polycyclic code when the Hamming distance of the residue code is known.

We use the above results to study some constacyclic codes of length $\eta p^{s}$ and $2 \eta p^{s}$ over $G R\left(p^{a}, m\right)$. First we compute the Hamming distance of these codes over the residue field. Then, we give the ideal structure and the Hamming distance of these codes by using a generating set in standard form. In some cases, our results give the Hamming distance of all such constacyclic codes.

As another application of our results, we generalize a recent result of [18] on the Hamming distance of cyclic codes of length $2^{s}$ over $\mathbb{Z}_{4}$. We classify all polycyclic codes over $G R\left(p^{2}, m\right)$ which gives us a classification of all cyclic codes of length $p^{s}$. Then we determine the torsional degrees of these codes in each case yielding the Hamming distance of all cyclic codes of length $p^{s}$ over $G R\left(p^{a}, m\right)$.

This paper is organized as follows. In Section 2, we give some preliminaries and fix our notation. In Section 3, we study the subambient rings of polycyclic codes along with their torsional degrees and strong 
Groebner bases. We further study these subambients in characteristic $p^{2}$ and determine their torsional degrees and Hamming distance in Section 4. We study the structure of the ambient ring of polycyclic codes in Section 5. We give some preliminaries for the computation of the Hamming distance of some constacyclic codes over a finite field in Section 6. Then we compute the Hamming distance of certain constacyclic codes of length $\eta p^{s}$ over $\mathbb{F}_{p^{m}}$ and we describe how to determine the Hamming distance of these codes over $G R\left(p^{a}, m\right)$ in Section 7 . Finally, in Section 8 , we carry out similar computations for certain constacyclic codes of length $2 \eta p^{s}$.

\section{Algebraic Background}

In this section we state some basic facts about finite chain rings, polynomials over Galois rings and we fix our notation on cyclic and polycyclic codes. For a detailed treatment of the theory of Galois rings, we refer the reader to [3] or [24].

Let $p$ be a prime number and $a, m \geq 1$ be integers. Then $\mathbb{F}_{p^{m}}$ denotes the finite field with $p^{m}$ elements and $G R\left(p^{a}, m\right)$ denotes the Galois ring of characteristic $p^{a}$ with $p^{a m}$ elements.

Let $R$ be a commutative ring with a unit. $R$ is called a local ring if it has a unique maximal ideal. An element $r \in R$ is said to be nilpotent with nilpotency index $t$ if $r^{t}=0$ and $t$ is the least nonnegative integer with respect to this property. The intersection of all maximal ideals of $R$ is called the Jacobson of $R$ and is denoted by $J(R)$. The socle of $R$, denoted by $\operatorname{soc}(R)$, is the sum of all ideals of $R$ containing only themselves and the zero ideal. $R$ is called a chain ring if its ideals are linearly ordered under set inclusion. In [14, a useful characterization of finite chain rings is given.

Lemma 2.1 ([14, Proposition 2.1]). Let $R$ be a finite commutative ring. The following are equivalent.

(1) $R$ is a chain ring.

(2) $R$ is a local principal ideal ring.

(3) $R$ is a local ring and the maximal ideal of $R$ is principal.

Furthermore, if $R$ is a finite commutative chain ring with the maximal ideal $\langle\nu\rangle$, then the ideals of $R$ are exactly $\left\langle\nu^{i}\right\rangle$ where $i \in\{0,1, \ldots, t\}$ and $t$ is the nilpotency index of $\nu$.

It is well-known that the Galois ring $G R\left(p^{a}, m\right)$ is a local ring with the maximal ideal $\langle p\rangle$. Moreover $G R\left(p^{a}, m\right)$ is a finite chain ring and its ideals are $\left\langle p^{i}\right\rangle$ where $i \in\{0,1, \ldots, a\}$. Let $\zeta$ be a generator of the multiplicative group $\mathbb{F}_{p^{m}} \backslash\{0\}$. The fact that $\mathbb{Z}_{p^{a}}[\zeta] \cong G R\left(p^{a}, m\right)$ is a classical result of finite ring theory. We can express an element $z \in G R\left(p^{a}, m\right)$ as $z=\sum_{j=0}^{p^{m}-2} v_{j} \zeta^{j}$ where $v_{j} \in \mathbb{Z}_{p^{a}}$. Let $\mathcal{T}_{m}=\left\{0,1, \zeta, \ldots, \zeta^{p^{m}-2}\right\}$. The set $\mathcal{T}_{m}$ is called the Teichmüller set. Alternatively, we can uniquely express $z \in G R\left(p^{a}, m\right)$ as

$$
z=z_{0}+p z_{1}+\cdots+p^{a-1} z_{a-1}, \quad z_{i} \in \mathcal{T}_{m},
$$

which is called the $p$-adic expansion of $z$. The map $\mu: \quad G R\left(p^{a}, m\right) \rightarrow \mathbb{F}_{p^{m}}$ defined by $\mu(z)=z_{0}$ is a ring epimorphism with the kernel $\langle p\rangle$. Hence $\frac{G R\left(p^{a}, m\right)}{\langle p\rangle} \cong \mathbb{F}_{p^{m}}$. The finite field $\mathbb{F}_{p^{m}}$ is called the residue field of $G R\left(p^{a}, m\right)$. The map $\mu$ is called the canonical projection and extends to a homomorphism between the polynomial rings $G R\left(p^{a}, m\right)[x]$ and $\mathbb{F}_{p^{m}}[x]$ in a natural way as $\mu\left(a_{0}+a_{1} x+\cdots+a_{n} x^{n}\right)=$ $\mu\left(a_{0}\right)+\mu\left(a_{1}\right) x+\cdots+\mu\left(a_{n}\right) x^{n}$. We denote $\mu(f(x))$ by $\bar{f}(x)$. Note also that $\mu$ maps the ideals of $G R\left(p^{a}, m\right)[x]$ to the ideals of $\mathbb{F}_{p^{m}}[x]$ and we denote the canonical projection of the ideal $I$ by $\bar{I}$.

A polynomial $f(x) \in G R\left(p^{a}, m\right)[x]$ is called regular if $f(x)$ is not a zero divisor. Moreover, by the characterization given in [24, Theorem XIII.2], $f(x)$ is regular if and only if one of its coefficients is a 
unit in $G R\left(p^{a}, m\right)$. If $f(x)$ can not be expressed as a product of two nonconstant polynomials, then $f(x)$ is called irreducible and if in addition $\bar{f}(x)$ is irreducible then $f(x)$ is called basic irreducible.

An ideal $I \triangleleft R$ is called a primary ideal if for all $u v \in I$, we have $u^{n} \in I$ or $v \in I$ for some positive integer $n$. A polynomial $f(x)$ is called primary if $\langle f(x)\rangle$ is a primary ideal. Besides, $I \triangleleft R$ is called a prime ideal if for all $u v \in I$, we have $u \in I$ or $v \in I$.

Theorem 2.2 ([24, Theorem XIII.11]). Let $f(x) \in G R\left(p^{a}, m\right)[x]$ be a regular polynomial. Then $f(x)=$ $\delta g_{1}(x) \cdots g_{r}(x)$ where $\delta$ is a unit and $g_{1}(x), \ldots, g_{r}(x)$ are regular primary coprime polynomials. Moreover, this factorization is unique up to reordering terms and multiplication by units.

Now we recall the division algorithm in $\mathbb{F}_{p^{m}}[x]$ and $G R\left(p^{a}, m\right)[x]$. Since $\mathbb{F}_{p^{m}}[x]$ is a Euclidean domain, for any $v(x)$ and $0 \neq g(x) \in \mathbb{F}_{p^{m}}[x]$, there exist unique polynomials $y(x), r(x) \in \mathbb{F}_{p^{m}}[x]$ such that

$$
v(x)=g(x) y(x)+r(x)
$$

where either $0 \leq \operatorname{deg}(r(x))<\operatorname{deg}(g(x))$ or $r(x)=0$. We define $v(x) \bmod g(x)=r(x)$, and we use the notation $v(x) \equiv r(x) \bmod g(x)$ in the usual sense.

There is also a division algorithm for polynomials in $G R\left(p^{a}, m\right)[x]$ (see, for example, [24, Exercise XIII.6] or [3, Proposition 3.4.4]). Let $f(x) \in G R\left(p^{a}, m\right)[x]$ and let $h(x) \in G R\left(p^{a}, m\right)[x]$ be a regular polynomial. Then there exist polynomials $z(x), b(x) \in G R\left(p^{a}, m\right)[x]$ such that

$$
f(x)=z(x) h(x)+b(x)
$$

and $\operatorname{deg}(b(x))<\operatorname{deg}(h(x))$ or $b(x)=0$.

Throughout this paper, $C$ stands for a linear code over $G R\left(p^{a}, m\right)$ and we identify a codeword $c=$ $\left(c_{0}, c_{1}, \ldots, c_{N-1}\right) \in C$ with the polynomial $c(x)=c_{0}+c_{1} x+\cdots+c_{N-1} x^{N-1} \in G R\left(p^{a}, m\right)[x]$. Let $\lambda \in$ $G R\left(p^{a}, m\right) \backslash\{0\}$ and $I=\left\langle x^{N}-\lambda\right\rangle$. The $\lambda$-shift of a codeword $c$ is defined to be $\left(\lambda c_{N-1}, c_{0}, c_{1}, \cdots, c_{N-2}\right)$. If a linear code $C$ is closed under $\lambda$-shifts, then $C$ is called a $\lambda$-cyclic code and in general, such codes are called constacyclic codes (c.f. [2, Section 13.2]). It is well-known that $\lambda$-cyclic codes, of length $N$, over $G R\left(p^{a}, m\right)$ correspond to the ideals of the finite ring

$$
\mathcal{R}_{c}=\frac{G R\left(p^{a}, m\right)[x]}{I} .
$$

In particular, cyclic (respectively negacyclic) codes, of length $N$, over $G R\left(p^{a}, m\right)$ correspond to the ideals of the ring $\mathcal{R}_{\mathfrak{a}}=G R\left(p^{a}, m\right)[x] / \mathfrak{a}$ (respectively $\mathcal{R}_{\mathfrak{b}}=G R\left(p^{a}, m\right)[x] / \mathfrak{b}$ ), where $\mathfrak{a}=\left\langle x^{N}-1\right\rangle$ (respectively $\left.\mathfrak{b}=\left\langle x^{N}+1\right\rangle\right)$. Additionally if $N$ is not divisible by $p$, then $C$ is called a simple-root constacyclic code and if $N$ is divisible by $p$, then $C$ is said to be a repeated-root constacyclic code.

Now we define a family of linear codes which is a generalization of constacyclic codes. Let $f(x) \in$ $G R\left(p^{a}, m\right)[x]$ be an arbitrary regular polynomial, $J=\langle f(x)\rangle$ and let

$$
\mathcal{R}=\frac{G R\left(p^{a}, m\right)[x]}{J} .
$$

As done above, identifying the codewords with polynomials, we see that the ideals of $\mathcal{R}$ are linear codes and they are called polycyclic codes. Obviously, although the elements of $\mathcal{R}$ are equivalence classes (cosets), they can be uniquely identified with polynomials with degree strictly less than $\operatorname{deg} f(x)$. Consequently, for the rest of this paper, unless otherwise stated, we focus on the ideals of $\mathcal{R}$ containing $J$ and identify $I / J$ with $\{g(x): \quad g(x) \in I$ and $\operatorname{deg}(g(x)<\operatorname{deg}(f(x)))\}$ and, for all $g(x)$ such that $\operatorname{deg}(g(x))<\operatorname{deg}(f(x))$, we identify the equivalence class $g(x)+J$ with $g(x)$. 
Let $\overline{\mathcal{R}}=\frac{\mathbb{F}_{p} m[x]}{J}$. The map $\mu$, defined above, extends to an onto ring homomorphism as $\mu: \mathcal{R} \rightarrow \overline{\mathcal{R}}$ where $\mu(g(x)+J)=\bar{g}(x)+\bar{J}$. For $r \in \mathcal{R}$ and $w \in \overline{\mathcal{R}}$, we define the scalar multiplication by $\operatorname{rw}(\bmod p)$ where we consider the multiplication in $\mathcal{R}$. This makes $\overline{\mathcal{R}}$ an $\mathcal{R}$-module.

The Hamming weight of a word is defined to be the number of nonzero entries of the word and the Hamming weight of a polynomial is defined to be the number of nonzero coefficients of the polynomial. Let $c$ and $c(x)$ be as above. We denote the Hamming weight of $c$ and $c(x)$ by $w_{H}(c)$ and $w_{H}(c(x))$, respectively. Obviously, the Hamming weight of a codeword and the Hamming weight of the corresponding polynomial are equal, i.e., $w_{H}(c)=w_{H}(c(x))$.

The Hamming distance of a linear code $C$ is defined as

$$
d_{H}(C)=\min \left\{w_{H}(v): \quad 0 \neq v \in C\right\} .
$$

The following lemma gives us some useful information on $d_{H}(C)$.

Lemma 2.3. Let $\{0\} \neq C \triangleleft \mathcal{R}$ be a constacyclic code of length greater than 1 over $G R\left(p^{a}, m\right)$ with $C \neq\{0\}$ and $C \neq\langle 1\rangle$, and let $\bar{C} \triangleleft \overline{\mathcal{R}}$ be its canonical projection. Then $d_{H}(C)=d_{H}(\bar{C})$ as the $\mathcal{R}$-modules $p^{a-1} \mathcal{R}$ and $\overline{\mathcal{R}}$ are isomorphic. Moreover $d_{H}(\bar{C}), d_{H}(C) \geq 2$.

Proof. The isomorphism is established by sending $f(x) \in \overline{\mathcal{R}}$ to $p^{a-1} f(x) \in p^{a-1} \mathcal{R}$. The bound $d_{H}(\bar{C}), d_{H}(C) \geq$ 2 follows from the facts that $d_{H}(C)=d_{H}(\bar{C})$ and a proper ideal can not contain a unit.

\section{LOCAL SUBAMBIENTS OF POLYCYCLIC CODES}

In this section, the ring

$$
\mathcal{R}=\frac{G R\left(p^{a}, m\right)[x]}{\langle f(x)\rangle},
$$

where $f(x) \in G R\left(p^{a}, m\right)[x]$ is a regular primary polynomial which is not a unit, is studied. The results of this section will be used to study the more general case, where $f(x)$ is not necessarily primary in Section 5.

First we show that $\mathcal{R}$ is a local ring and determine its maximal ideal, we determine the socle of $\mathcal{R}$, for $a \geq 1$, we give necessary and sufficient conditions for $\mathcal{R}$ to be a chain ring in Lemma 3.4. Then, using the notion of torsional code and torsional degree, we determine a unique generating set for any ideal of $\mathcal{R}$ in Theorem 3.11. Next we observe, in Corollary 3.13, that such a generating set is a strong Groebner basis and if we remove the redundant generators, we obtain a generating set in standard form which is a minimal strong Groebner basis. Finally, we show that the torsional degrees of a polycyclic code can immediately be obtained from a generating set in standard form.

In this section we assume $f(x)$ is a regular primary polynomial that is not a unit. By [24, Theorem XIII.6], $f(x)=v f^{*}(x)$ where $v$ is a unit and $f^{*}(x)$ is monic and regular. Since $\langle f(x)\rangle=\left\langle v f^{*}(x)\right\rangle$ and because of our interest in $\mathcal{R}$, assume $f(x)$ is monic. By Proposition [24, XIII.12], $f(x)=\delta(x) h(x)^{t}+p \beta(x)$ for some $\delta(x), h(x), \beta(x) \in G R\left(p^{a}, m\right)[x]$ where $\delta(x)$ is a unit and $h(x)$ is a basic irreducible polynomial. Since $\delta(x)$ is a unit, by [24, Theorem XIII.2], $\delta(x)=\delta_{0}+p \delta^{\prime}(x)$ for some $\delta_{0} \in G R\left(p^{a}, m\right)$ that is a unit and some $\delta^{\prime}(x) \in G R\left(p^{a}, m\right)[x]$. Also, since $h(x)$ is basic, $h(x)=\bar{h}(x)+p \alpha(x)$ for some $\alpha(x) \in G R\left(p^{a}, m\right)[x]$. So, $\bar{f}(x)=\delta_{0} \bar{h}(x)^{t}$ and $f(x)=\delta_{0} \bar{h}(x)^{t}+p \beta^{\prime}(x)$ for some $\beta^{\prime}(x) \in G R\left(p^{a}, m\right)[x]$.

Assume $f(x)=\delta h(x)^{t}+p \beta(x)$ where $\delta \in G R\left(p^{a}, m\right)$ is a unit and $h(x)$ is a basic irreducible such that $\bar{h}(x)=h(x)$. By the fact that $f(x)$ is monic, we know that $t \operatorname{deg} h(x)>\operatorname{deg} \beta(x)$. Furthermore, without loss of generality, we may assume $h(x)$ is monic. By this assumption, $\delta=1$ since $f(x)$ is monic. Hence, 
$f(x)$ is a monic regular primary polynomial such that $f(x)=h(x)^{t}+p \beta(x)$ where $h(x)$ is a monic basic irreducible polynomial such that $\bar{h}(x)=h(x)$.

We show that $\langle p, h(x)\rangle$ is the unique maximal ideal of $\mathcal{R}$.

Lemma 3.1. The ring $\mathcal{R}$ is local with maximal ideal $J(\mathcal{R})=\langle p+\langle f\rangle, h(x)+\langle f\rangle\rangle$.

Proof. As discussed in page 262 of [24], any maximal ideal in $G R\left(p^{a}, m\right)[x]$ is of the form $\langle p, g(x)\rangle$ where $g(x)$ is a basic irreducible polynomial. Assume $f(x) \in\langle p, g(x)\rangle$ where $g(x) \in G R\left(p^{a}, m\right)[x]$ is a basic irreducible polynomial. Then for some $a(x), b(x) \in G R\left(p^{a}, m\right)[x]$

$$
\begin{aligned}
f(x) & =a(x) p+b(x) g(x), \\
\bar{f}(x) & =\bar{b}(x) \bar{g}(x), \\
\bar{h}(x)^{t} & =\bar{b}(x) \bar{g}(x) .
\end{aligned}
$$

This shows that $\bar{h}(x) \mid \bar{g}(x)$ which implies $\bar{g}(x) \mid \bar{h}(x)$ and $g(x)=h(x)+p c(x)$ for some $c(x) \in G R\left(p^{a}, m\right)[x]$. So, $\langle p, g(x)\rangle=\langle p, h(x)\rangle$ meaning $\langle p, h(x)\rangle$ is the only maximal ideal containing $f(x)$. Hence, $\langle p+\langle f\rangle, h(x)+\langle f\rangle\rangle$ is the unique maximal ideal of $\mathcal{R}$.

In the case of finite fields, $\mathcal{R}$ is a chain ring.

Lemma 3.2. The quotient ring $\frac{G R(p, m)[x]}{\langle f(x)\rangle}$ is a chain ring with exactly the following ideals

$$
\frac{G R(p, m)[x]}{\langle f(x)\rangle}=\left\langle h(x)^{0}+\langle f\rangle\right\rangle \supsetneq\left\langle h(x)^{1}+\langle f\rangle\right\rangle \supsetneq \cdots \supsetneq\left\langle h(x)^{t}+\langle f\rangle\right\rangle=0 .
$$

Proof. By Lemma 3.1, $\frac{G R(p, m)[x]}{\langle f(x)\rangle}$ is local with $J\left(\frac{G R(p, m)[x]}{\langle f(x)\rangle}\right)=\langle h(x)+\langle f\rangle\rangle$. By Lemma 2.1, the result follows.

Now we determine the socle of $\mathcal{R}$ and show that it is simple.

Lemma 3.3. The ring $\mathcal{R}$ has simple socle with $\operatorname{soc}(\mathcal{R})=\left\langle p^{a-1} h(x)^{t-1}+\langle f\rangle\right\rangle$.

Proof. Let $g(x)+\langle f\rangle \in \mathcal{R}$. Let $\ell$ be the largest integer such that $p^{\ell}(g(x)+\langle f\rangle) \neq 0$. By Lemma 3.1, $J(\overline{\mathcal{R}})=\langle h(x)+\langle f\rangle\rangle$. By Lemma 2.3 and Lemma 3.2 and the fact that $p^{\ell}(g(x)+\langle f\rangle) \in\left\langle p^{a-1}+\langle f\rangle\right\rangle$, it can be shown that $\left\langle p^{a-1} h(x)^{t-1}+\langle f\rangle\right\rangle \subset\langle g(x)+\langle f\rangle\rangle$. So $\left\langle p^{a-1} h(x)^{t-1}+\langle f\rangle\right\rangle$ is contained in any principal ideal. Since $J(\mathcal{R})$ annihilates $\left\langle p^{a-1} h(x)^{t-1}+\langle f\rangle\right\rangle$, $\operatorname{soc}(\mathcal{R})=\left\langle p^{a-1} h(x)^{t-1}+\langle f\rangle\right\rangle$. It is clearly simple.

Lemma 3.2 tells us when the alphabet is a finite field, then $\mathcal{R}$ is a chain ring. However, $\mathcal{R}$ is not a chain ring in general. As a counter example, consider $\frac{\mathbb{Z}_{4}[x]}{\left\langle x^{2}-1\right\rangle}$. We have $x^{2}-1=(x+1)^{2}-2(x+1)$. Clearly, $(x+1) \notin\langle 2\rangle$ in $\frac{\mathbb{Z}_{4}[x]}{\left\langle x^{2}-1\right\rangle}$. Assume $2 \in\langle x+1\rangle$. Then $2=g_{1}(x)(x+1)+g_{2}(x)\left(x^{2}-1\right) \in \mathbb{Z}_{4}[x]$. Evaluating at $x=-1$, we get $2=0$ in $\mathbb{Z}_{4}$. This is a contradiction. Thus we have shown $\langle 2\rangle \not \subset\langle x+1\rangle$ and $\langle x+1\rangle \not \subset\langle 2\rangle$. By Lemma 3.1, $J\left(\frac{\mathbb{Z}_{4}[x]}{\left\langle x^{2}-1\right\rangle}\right)=\langle 2, x+1\rangle$. Since $J\left(\frac{\mathbb{Z}_{4}[x]}{\left\langle x^{2}-1\right\rangle}\right)$ is 2-generated, by Lemma $2.1 \frac{\mathbb{Z}_{4}[x]}{\left\langle x^{2}-1\right\rangle}$ is not a chain ring.

The next theorem shows exactly when $\mathcal{R}$ is a chain ring based on the parameters $a, t, h(x)$ and $\beta(x)$ of $f(x)$.

Theorem 3.4. The ring $\mathcal{R}$ is a chain ring if and only if any one of the conditions is met

(1) $a=1$

(2) $t=1$ 
(3) $\beta(x) \notin\langle p, h(x)\rangle$.

Proof. Assume $a=1$. By Lemma $3.2, \mathcal{R}$ is a chain ring.

Assume $t=1$ then $h(x)=f(x)-p \beta(x) \in\langle p, f(x)\rangle$. So, $h(x)+\langle f\rangle \in\langle p+\langle f\rangle\rangle$. By Lemma 3.1, $J(\mathcal{R})=\langle p+\langle f\rangle\rangle$. Hence, by Lemma 2.1, $\mathcal{R}$ is a chain ring.

Assume $\beta(x) \notin\langle p, h(x)\rangle$. Then $\beta(x)+\langle f\rangle \notin J(\mathcal{R})$ which implies $\beta(x)+\langle f\rangle$ is a unit in $\mathcal{R}$. So, $\langle p+\langle f\rangle\rangle=\left\langle h(x)^{t}+\langle f\rangle\right\rangle$ which implies $p+\langle f\rangle \in\langle h(x)+\langle f\rangle\rangle$. By Lemma 3.1, $J(\mathcal{R})=\langle h(x)+\langle f\rangle\rangle$. Hence, by Lemma 2.1, $\mathcal{R}$ is a chain ring.

Now assume $a>1, t>1$ and $\beta(x) \in\langle p, h(x)\rangle$. We want to show that $\mathcal{R}$ is not a chain ring so assume the contrary. This implies $\langle p+\langle f\rangle\rangle \subset\langle h(x)+\langle f\rangle\rangle$ or $\langle h(x)+\langle f\rangle\rangle \subset\langle p+\langle f\rangle\rangle$. So, $p \in\langle h(x), f(x)\rangle$ or $h(x) \in\langle p, f(x)\rangle$. First, assume $p \in\langle h(x), f(x)\rangle$ which implies $\beta(x) \in\langle p, h(x)\rangle=\langle p, h(x), f(x)\rangle=$ $\langle h(x), f(x)\rangle$. So,

$$
f(x)=h(x)^{t}+p \beta(x)=h(x)^{t}+p(\gamma(x) h(x)+\alpha(x) f(x))
$$

for some $\gamma(x), \alpha(x) \in G R\left(p^{a}, m\right)[x]$ and

$$
f(x)(1-p \alpha(x))=h(x)\left(h(x)^{t-1}+p \gamma(x)\right) .
$$

Since $(1-p \alpha(x))$ is invertible in $G R\left(p^{a}, m\right)[x], f(x) \in\langle h(x)\rangle$. So, $p \in\langle h(x), f(x)\rangle=\langle h(x)\rangle$. Since $a>1$, $p \neq 0$. This is a contradiction since $p$ cannot be a nonzero multiple of $h(x)$.

Next, assume $h(x) \in\langle p, f(x)\rangle$. Then,

$$
h(x)^{t}=[\gamma(x) p+\alpha(x) f(x)]^{t}=f(x)-p \beta(x)
$$

for some $\gamma(x), \alpha(x) \in G R\left(p^{a}, m\right)[x]$. This implies,

$$
\overline{[\alpha(x) f(x)]^{t}}=\overline{f(x)} \text {. }
$$

Since $t>1$, by comparing degrees we see this is a contradiction. Hence, $\mathcal{R}$ is not a chain.

Below are two examples that show the distinctions between the particular cases in Theorem 3.4 .

Example 3.5. Let $a>1, p=2, s>0$ and $f(x)=x^{2^{s}}+1$. Then

$$
\begin{aligned}
x^{2^{s}}+1 & =(x+1-1)^{2^{s}}+1 \\
& =(x+1)^{2^{s}}-\left(\begin{array}{c}
2^{s} \\
2^{s}-1
\end{array}\right)(x+1)^{2^{s}-1}+\cdots-\left(\begin{array}{c}
2^{s} \\
1
\end{array}\right)(x+1)+1+1 \\
& =(x+1)^{2^{s}}+2 \beta(x)
\end{aligned}
$$

where $\beta(x)=(x+1) q(x)+1$ for some $q(x) \in \mathcal{R}$. In [8] it was shown that $\frac{G R\left(p^{a}, m\right)[x]}{\langle f(x)\rangle}$ is a chain ring with the maximal ideal $\langle x+1\rangle$.

Example 3.6. Let $a>1, p=2, s>0$ and $f(x)=x^{2^{s}}-1$. Then

$$
\begin{aligned}
x^{2^{s}}-1 & =(x+1-1)^{2^{s}}-1 \\
& =(x+1)^{2^{s}}-\left(\begin{array}{c}
2^{s} \\
2^{s}-1
\end{array}\right)(x+1)^{2^{s}-1}+\cdots-\left(\begin{array}{c}
2^{s} \\
1
\end{array}\right)(x+1)+1-1 \\
& =(x+1)^{2^{s}}+2 \beta(x)
\end{aligned}
$$

where $(x+1) \mid \beta(x)$. In [22] it was shown that $\frac{G R\left(p^{a}, m\right)[x]}{\langle f(x)\rangle}$ is local with the maximal ideal $\langle 2,(x+1)\rangle$ and is not a chain ring. 
Theorem 3.4 shows that $\mathcal{R}$ is not a principal ideal ring in general. Through the next series of results we will show the existence of a particular generating set which turns out to be a strong Groebner basis.

Let $g(x) \in G R\left(p^{a}, m\right)[x]$ and $n$ be the largest integer such that $\operatorname{deg}(g(x)) \geq n \operatorname{deg}(h(x))$. By the division algorithm, we can find $q_{n}(x), r_{1}(x) \in G R\left(p^{a}, m\right)[x]$ such that

$$
g(x)=q_{n}(x) h(x)^{n}+r_{1}(x),
$$

where $r_{1}(x)=0$ or $\operatorname{deg}\left(r_{1}(x)\right)<n \operatorname{deg}(h(x))$. Note that $\operatorname{deg}\left(q_{n}(x)\right)<\operatorname{deg}(h(x))$. Next we can find $q_{n-1}(x), r_{2}(x) \in G R\left(p^{a}, m\right)[x]$ such that

$$
r_{1}(x)=q_{n-1}(x) h(x)^{n-1}+r_{2}(x)
$$

where $r_{2}(x)=0$ or $\operatorname{deg}\left(r_{2}(x)\right)<(n-1) \operatorname{deg}(h(x))$. Note that $\operatorname{deg}\left(q_{n-1}(x)\right)<\operatorname{deg}(h(x))$. We can continue this process until we have $q_{n}(x), q_{n-1}(x), \ldots, q_{0}(x) \in G R\left(p^{a}, m\right)[x]$ where

$$
g(x)=q_{n}(x) h(x)^{n}+\cdots+q_{1}(x) h(x)+q_{0}(x)
$$

where for $0 \leq i \leq n$, either $\operatorname{deg}\left(q_{i}(x)\right)<\operatorname{deg}(h(x))$ or $q_{i}(x)=0$. With some manipulation $g(x)$ can be represented in the following form

$$
g(x)=p^{j_{0}} h(x)^{i_{0}} \alpha_{0}(x)+\cdots+p^{j_{r}} h(x)^{i_{r}} \alpha_{r}(x)
$$

where $0 \leq r \leq a-1$ and

- $\alpha_{i}(x) \notin\langle p, h(x)\rangle$

- $0 \leq j_{0}<\cdots<j_{r} \leq a-1$

- $i_{0}>\cdots>i_{r} \geq 0$.

Since $f(x)$ is regular and monic, $g(x)$ can be divided by $f(x)$ initially. Then it is not hard to see that for some $q(x) \in G R\left(p^{a}, m\right)[x]$

$$
g(x)=q(x) f(x)+p^{j_{0}} h(x)^{i_{0}} \alpha_{0}(x)+\cdots+p^{j_{r}} h(x)^{i_{r}} \alpha_{r}(x)
$$

where $r, \alpha_{i}(x), j_{e}$ and $i_{\ell}$ are as above with $t>i_{0}$.

In [15] and [20], a unique generating set for an ideal of $\frac{G R\left(p^{a}, m\right)[x]}{\left\langle x^{p^{s}}-1\right\rangle}$ was developed. The polynomial $x^{p^{s}}-1$ is of the type $f(x)$ is. Notice $x^{p^{s}}-1=(x-1)^{p^{s}}+p \beta(x)$. We will now find a similar generating set for an ideal of $\mathcal{R}$.

Definition 3.7 (cf. [15, Definition 6.1]). Let $C \triangleleft \mathcal{R}$. For $0 \leq i \leq a-1$, define

$$
\operatorname{Tor}_{i}(C)=\left\{\mu(v): \quad p^{i} v \in C\right\} .
$$

$\operatorname{Tor}_{i}(C)$ is called the $i^{\text {th }}$ torsion code of $C$. $\operatorname{Tor}_{0}(C)=\mu(C)$ is usually called the residue code of $C$. Note that for a code $C$ over $G R\left(p^{a}, m\right)$, we have $\operatorname{Tor}_{i}(C) \subset \operatorname{Tor}_{i+1}(C)$.

Lemma 3.8. Let $C \triangleleft \mathcal{R}$. Then

$$
\operatorname{Tor}_{i}(C)=\left\langle h(x)^{T_{i}}+\langle f\rangle\right\rangle \subset \frac{G R(p, m)[x]}{\langle f(x)\rangle}
$$

for some $0 \leq T_{i} \leq t$.

Proof. Since $C \triangleleft \mathcal{R}, \operatorname{Tor}_{i}(C) \triangleleft \frac{G R(p, m)[x]}{\langle\bar{f}(x)\rangle}$. The claim follows by Lemma 3.2.

Definition 3.9. In Lemma 3.8, $T_{i}$ is the $i^{\text {th }}$ torsional degree of $C$ which we denote by $T_{i}(C)$. The torsional degrees form a non-increasing sequence, i.e., $t \geq T_{0}(C) \geq \cdots \geq T_{a-1}(C) \geq 0$. 
For any $\xi(x)+\langle f\rangle \in \mathcal{R}$, we can divide $\xi(x)$ by $f(x)$, as $f(x)$ is regular, and get $\xi(x)=q(x) f(x)+r(x)$ such that either $r(x)=0$ or $\operatorname{deg}(r(x))<\operatorname{deg}(f(x))$. So $\xi(x)+\langle f\rangle=r(x)+\langle f\rangle$. This implies that $\mathcal{R}=\left\{a(x)+\langle f\rangle: a(x) \in G R\left(p^{a}, m\right)[x], \operatorname{deg}(a(x))<\operatorname{deg}(f(x))\right\}$. Throughout the remainder for this section, the elements of $\mathcal{R}$ will be represented as polynomials of degree less than $\operatorname{deg}(f(x))$.

Definitions 3.7 and 3.9 and Lemma 3.8 are expansions to polycyclic codes of the ideas first presented in Section 6 of [15] in the context of cyclic codes. The following theorem is a generalization of Theorem 6.5 of [15.

Theorem 3.10. Let $C \triangleleft \mathcal{R}$. Then $C=\left\langle F_{0}(x), p F_{1}(x), \ldots, p^{a-1} F_{a-1}(x)\right\rangle$ where $F_{i}(x)=0$ if $T_{i}(C)=t$, and $F_{i}(x)=h(x)^{T_{i}(C)}+p \gamma_{i}(x)$ for some $\gamma_{i}(x) \in G R\left(p^{a}, m\right)[x]$, if $T_{i}(C)<t$.

Proof. Denote $T_{i}(C)$ by $T_{i}$. If $C=0$, we are done. So assume $C \neq 0$. Let $r$ be the smallest nonnegative integer such that $T_{r}<t$. For every $0 \leq i \leq r-1$, set $F_{i}(x)=0$. For $r \leq i \leq a-1$, pick $F_{i}(x) \in$ $G R\left(p^{a}, m\right)[x]$ such that $p^{i} F_{i}(x) \in C$ and $\mu\left(F_{i}(x)\right)=h(x)^{T_{i}}$. So, $F_{i}(x)=h(x)^{T_{i}}+p \gamma_{i}(x)$ for some $\gamma_{i}(x) \in \mathcal{R}$. Note that such an $F_{i}(x)$ exists because $\operatorname{Tor}_{i}(C)=\left\langle h(x)^{T_{i}}\right\rangle \triangleleft \frac{G R(p, m)[x]}{\langle f(x)\rangle}$. Let $g(x) \in C$. As was shown earlier (see Equation (3.1)),

$$
g(x)=p^{j_{0}}\left(h(x)^{i_{0}} \sigma_{j_{0}}(x)+p \beta_{0}(x)\right)
$$

for some $\sigma_{j_{0}}(x), \beta_{0}(x) \in G R\left(p^{a}, m\right)[x]$ where $i_{0}<t$ and $\sigma_{j_{0}}(x) \neq 0$. Let $\sigma_{0}(x)=\cdots=\sigma_{j_{0}-1}(x)=0$. Let

$$
g_{1}(x)=g(x)-p^{j_{0}} h(x)^{i_{0}-T_{j_{0}}} \sigma_{j_{0}}(x) F_{j_{0}}(x) .
$$

Note that since $\operatorname{Tor}_{j_{0}}(C)=\left\langle h(x)^{T_{j_{0}}}\right\rangle$, it follows by (3.2) and the fact that $\sigma_{j_{0}}(x)$ is a unit in $\frac{G R\left(p^{a}, m\right)[x]}{\langle f(x)\rangle}$ that $i_{0} \geq T_{j_{0}}$. Since $T_{j_{0}}<t$, we have

$$
\begin{aligned}
g_{1}(x) & =p^{j_{0}}\left(h(x)^{i_{0}} \sigma_{j_{0}}(x)+p \beta_{0}(x)\right)-p^{j_{0}} h(x)^{i_{0}-T_{j_{0}}} \sigma_{j_{0}}(x)\left[h(x)^{T_{j_{0}}}+p \gamma_{j_{0}}(x)\right] \\
& =p^{j_{0}+1} \beta_{0}(x)-p^{j_{0}+1} h(x)^{i_{0}-T_{j_{0}}} \sigma_{j_{0}}(x) \gamma_{j_{0}}(x) .
\end{aligned}
$$

So, $g_{1}(x) \in\left\langle p^{j_{0}+1}\right\rangle \cap C$. If $g_{1}(x)=0$, let $\sigma_{j_{0}+1}(x)=\cdots=\sigma_{a-1}(x)=0$ and we are done. If not, then, as was done with $g(x)$, we can view $g_{1}(x)$ as

$$
g_{1}(x)=p^{j_{1}}\left(h(x)^{i_{1}} \sigma_{j_{1}}(x)+p \beta_{1}(x)\right)
$$

for some $\sigma_{j_{1}}(x), \beta_{1}(x) \in G R\left(p^{a}, m\right)[x]$ where $i_{1}<t, j_{0}<j_{1}$ and $\sigma_{j_{1}}(x) \neq 0$. Let $\sigma_{j_{0}+1}(x)=\cdots=$ $\sigma_{j_{1}-1}(x)=0$. Let

$$
g_{2}(x)=g_{1}(x)-p^{j_{1}} h(x)^{i_{1}-T_{j_{1}}} \sigma_{j_{1}}(x) F_{j_{1}}(x)
$$

Since $T_{j_{1}}<t$, we have

$$
\begin{aligned}
g_{2}(x) & =p^{j_{1}}\left(h(x)^{i_{1}} \sigma_{j_{1}}(x)+p \beta_{1}(x)\right)-p^{j_{1}} h(x)^{i_{1}-T_{j_{1}}} \sigma_{1}(x)\left[h(x)^{T_{j_{1}}}+p \gamma_{j_{1}}(x)\right] \\
& =p^{j_{1}+2} \beta_{1}(x)-p^{j_{1}+1} h(x)^{i_{1}-T_{j_{1}}} \sigma_{1}(x) \gamma_{j_{1}}(x) .
\end{aligned}
$$

So $g_{2}(x) \in\left\langle p^{j_{1}+1}\right\rangle \cap C$. If $g_{2}(x)=0$, then let $\sigma_{j_{1}+1}(x)=\cdots=\sigma_{a-1}(x)=0$. Note that since $j_{0}<j_{1}<a$, this is a finite process. So

$$
g(x)=\sum_{i=0}^{a-1} p^{i} h(x)^{i-T_{i}} \sigma_{i}(x) F_{i}(x) \in\left\langle F_{0}(x), p F_{1}(x), \ldots, p^{a-1} F_{a-1}\right\rangle .
$$

Hence $C \subset\left\langle F_{0}(x), p F_{1}(x), \ldots, p^{a-1} F_{a-1}(x)\right\rangle$. Since $p^{i} F_{i}(x) \in C$, for all $0 \leq i \leq a-1$, we have the equality

$$
C=\left\langle F_{0}(x), p F_{1}(x), \ldots, p^{a-1} F_{a-1}(x)\right\rangle
$$


As was stated in [20], Theorem 6.5 of [15] does not provide a unique set of generators. Neither does our generalization in Theorem 3.10. We now show, as in [20], that there does exist a unique set of generators given some extra constraints. Although this is a generalization of Theorem 2.5 in [20], the proof here only differs from that one in a few details. However, we present the proof in its entirety here for the sake of completeness.

We would like to point out that there is a little inaccuracy in the statement of Theorem 2.5 in [20]. Let $\mathcal{T}_{m}[u]$ be the set of polynomials in $u$ whose coefficients are in $\mathcal{T}_{m}$. The $h_{j, \ell}(u)$ in their theorem is said to be an element of $\mathcal{T}_{m}[u]$ which is not necessarily true. What is true is that $h_{j, \ell}(u)$ is either 0 or a unit and that

$$
h_{j, \ell}(u)=\sum_{k=0}^{T_{\ell+j}-1} c_{k, j, \ell}(u-1)^{k}
$$

with $c_{k, j, \ell} \in \mathcal{T}_{m}$ and $c_{0, j, \ell} \neq 0$. It should also be pointed out that $h_{j, \ell}(u)$ is a unit precisely because $(u-1)$ is nilpotent (which is not stated but fairly easy to show) and $c_{0, j, \ell}$ is a unit.

Theorem 3.11. Let $C \triangleleft \mathcal{R}$. Then there exist $f_{0}(x), f_{1}(x), \ldots, f_{a-1}(x) \in \mathcal{R}$ such that

$$
C=\left\langle f_{0}(x), p f_{1}(x), \ldots, p^{a-1} f_{a-1}(x)\right\rangle
$$

where $f_{i}(x)=0$, if $T_{i}(C)=t$ otherwise

$$
f_{i}(x)=h(x)^{T_{i}(C)}+\sum_{j=1}^{a-1-i} p^{j} h(x)^{t_{i, j}} \alpha_{i, j}(x)
$$

where $t_{i, j} \operatorname{deg}(h(x))+\operatorname{deg}\left(\alpha_{i, j}(x)\right)<T_{i+j}(C) \operatorname{deg}(h(x))$ and each $\alpha_{i, j}(x) \notin\langle p, h(x)\rangle \backslash\{0\}$.

Furthermore, the set $\left\{f_{0}(x), p f_{1}(x), \ldots, p^{a-1} f_{a-1}(x)\right\}$ is the unique generating set with these properties.

Proof. Denote $T_{i}(C)$ by $T_{i}$. When $C=0$, the result holds. Assume $C \neq 0$. By Theorem 3.10, $C=$ $\left\langle F_{0}(x), p F_{1}(x), \ldots, p^{a-1} F_{a-1}(x)\right\rangle$ where $F_{i}(x)=0$ when $T_{i}=t$, otherwise $F_{i}(x)=h(x)^{T_{i}}+p \gamma_{i}(x)$ for some $\gamma_{i}(x) \in G R\left(p^{a}, m\right)[x]$. The torsional degrees of $C$ form the non-increasing sequence $t \geq T_{0} \geq \cdots \geq$ $T_{a-1} \geq 0$. Since $C \neq\{0\}$ there is a least positive integer $r$ such that $t>T_{r} \geq \cdots \geq T_{a-1} \geq 0$. For $0 \leq i \leq r-1, F_{i}(x)=0$. Let $f_{i}(x)=0$ for $0 \leq i \leq r-1$. For $r \leq i \leq a-1, F_{i}(x) \neq 0$. Since we are considering $p^{i} F_{i}(x)$ and $F_{i}(x)$ can be put in the form as shown in equation (3.1), without loss of generality we can write

$$
F_{i}(x)=h(x)^{T_{i}}+\sum_{j=1}^{a-1-i} p^{j} \sum_{k=0}^{t-1} h(x)^{k} q_{i, j, k}(x)
$$

where $q_{i, j, k}(x)=\sum_{l=0}^{\operatorname{deg} h-1} b_{i, j, k, l} x^{l}$ with $b_{i, j, k, l} \in \mathcal{T}_{m}$.

Let

$$
f_{a-1}(x)=F_{a-1}(x)=h(x)^{T_{a-1}} .
$$


Now,

$$
\begin{aligned}
F_{a-2}(x)= & h(x)^{T_{a-2}}+p \sum_{k=0}^{t-1} h(x)^{k} q_{a-2,1, k}(x) \\
= & h(x)^{T_{a-2}} \\
& +p\left[\sum_{k=0}^{T_{a-1}-1} h(x)^{k} q_{a-2,1, k}(x)+h(x)^{T_{a-1}} \sum_{k=T_{a-1}}^{t-1} h(x)^{k-T_{a-1}} q_{a-2,1, k}(x)\right] .
\end{aligned}
$$

Let

$$
\begin{aligned}
f_{a-2}(x) & =F_{a-2}(x)-p f_{a-1}(x) \sum_{k=T_{a-1}}^{t-1} h(x)^{k-T_{a-1}} q_{a-2,1, k}(x) \\
& =F_{a-2}(x)-p h(x)^{T_{a-1}} \sum_{k=T_{a-1}}^{t-1} h(x)^{k-T_{a-1}} q_{a-2,1, k}(x) \\
& =h(x)^{T_{a-2}}+p \sum_{k=0}^{T_{a-1}-1} h(x)^{k} q_{a-2,1, k}(x) \\
& =h(x)^{T_{a-2}}+p h(x)^{t_{a-2,1}} \sum_{k=t_{a-2,1}}^{T_{a-1-1}} h(x)^{k-t_{a-2,1}} q_{a-2,1, k}(x)
\end{aligned}
$$

where $t_{a-2,1}$ is the smallest $k$ such that $q_{a-2,1, k}(x) \neq 0$ if such a $k$ exists, otherwise $\sum_{k=t_{a-2,1}}^{T_{a-1}-1} h(x)^{k-t_{a-2, a-1}} q_{a-2,1, k}(x)=0$ and $t_{a-2,1}$ can be arbitrary. It is easy to see

$$
C=\left\langle F_{0}(x), p F_{1}(x), \ldots, p^{a-3} F_{a-3}(x), p^{a-2} f_{a-2}(x), p^{a-1} f_{a-1}(x)\right\rangle
$$

and that $f_{a-2}(x)$ and $f_{a-1}(x)$ satisfy the conditions in the theorem.

We proceed by induction. Assume $f_{i+1}(x), \ldots, f_{a-1}(x)$ satisfy the conditions of the theorem and that

$$
C=\left\langle F_{0}(x), p F_{1}(x), \ldots, p^{i} F_{i}(x), p^{i+1} f_{i+1}(x), \ldots, p^{a-1} f_{a-1}(x)\right\rangle .
$$

After subtracting appropriate multiples of $p^{i+1} f_{i+1}(x), \ldots, p^{a-1} f_{a-1}(x)$ from $F_{i}(x)$ we can find an element $f_{i}(x)$ such that

$$
\begin{aligned}
f_{i}(x) & =h(x)^{T_{i}}+\sum_{j=1}^{a-1-i} p^{j} \sum_{k=0}^{T_{i+j}-1} h(x)^{k} g_{i, j, k}(x) \\
& =h(x)^{T_{i}}+\sum_{j=1}^{a-1-i} p^{j} h(x)^{t_{i, j}} \sum_{k=t_{i, j}}^{T_{i+j}-1} h(x)^{k-t_{i, j}} g_{i, j, k}(x)
\end{aligned}
$$

where $g_{i, j, k}(x)=\sum_{l=0}^{\operatorname{deg} h-1} c_{i, j, k, l} x^{l}$ for some $c_{i, j, k, l} \in \mathcal{T}_{m}$ and for fixed $j, t_{i, j}$ is the smallest $k$ such that $g_{i, j, k}(x) \neq 0$ if such a $k$ exists, otherwise $\sum_{k=t_{i, j}}^{T_{i+j}-1} h(x)^{k-t_{i, j}} g_{i, j, k}(x)=0$ and $t_{i, j}$ can be arbitrary. Let $\alpha_{i, j}(x)=\sum_{k=t_{i, j}}^{T_{i+j}-1} h(x)^{k-t_{i, j}} g_{i, j, k}(x)$. If $\alpha_{i, j}(x) \neq 0, \alpha_{i, j}(x)$ is a unit since $\alpha_{i, j}(x) \notin\langle p, h(x)\rangle$. It is easy to see that

$$
C=\left\langle F_{0}(x), p F_{1}(x), \ldots, p^{i-1} F_{i-1}(x), p^{i} f_{i}(x), \ldots, p^{a-1} f_{a-1}(x)\right\rangle
$$

and $f_{i}(x), \ldots, f_{a-1}(x)$ satisfy the conditions in the theorem. Hence, we have $f_{0}(x), \ldots, f_{a-1}(x)$ such that

$$
C=\left\langle f_{0}(x), p f_{1}(x), \ldots, p^{a-1} f_{a-1}(x)\right\rangle .
$$


Now we show the uniqueness of such a generating set. Assume that $f_{0}^{\prime}(x), \cdots, f_{a-1}^{\prime}(x)$ also satisfy the conditions in the theorem. Say

$$
f_{i}(x)=h(x)^{T_{i}}+\sum_{j=1}^{a-1-i} p^{j} \sum_{k=0}^{T_{i+j}-1} h(x)^{k} g_{i, j, k}(x)
$$

and

$$
f_{i}^{\prime}(x)=h(x)^{T_{i}}+\sum_{j=1}^{a-1-i} p^{j} \sum_{k=0}^{T_{i+j}-1} h(x)^{k} g_{i, j, k}^{\prime}(x)
$$

where $g_{i, j, k}(x), g_{i, j, k}^{\prime}(x) \in \mathcal{T}_{m}[x]$ of degree less than $h(x)$. Assume $f_{i}(x)-f_{i}^{\prime}(x) \neq 0$. Then for some $j, k$, $g_{i, j, k}(x)-g_{i, j, k}^{\prime}(x) \neq 0$. Let $j_{0}$ be the smallest $j$ in the above sum such that $g_{i, j, k}(x)-g_{i, j, k}^{\prime}(x) \neq 0$. Then

$$
p^{i}\left(f_{i}(x)-f_{i}^{\prime}(x)\right)=p^{i+j_{0}} \sum_{j=j_{0}}^{a-1-i} p^{j-j_{0}} \sum_{k=0}^{T_{i+j}-1} h(x)^{k}\left(g_{i, j, k}(x)-g_{i, j, k}^{\prime}(x)\right) .
$$

Since the difference of two distinct elements of $\mathcal{T}_{m}$ is not divisible by $p$, for all $j, k$ in the above sum, either $g_{i, j, k}(x)-g_{i, j, k}^{\prime}(x)$ is 0 or not divisible by $p$. By the assumption on $j_{0}$ then, $p^{i}\left(f_{i}(x)-f_{i}^{\prime}(x)\right) \in$ $C \bigcap\left\langle p^{i+j_{0}}\right\rangle \backslash\left\langle p^{i+j_{0}+1}\right\rangle$. Since this is a nonzero element of $C$ with degree less than $T_{i+j_{0}} \operatorname{deg}(h)$, this contradicts the definition of $T_{i+j_{0}}$. Hence $f_{i}(x)=f_{i}^{\prime}(x)$.

Now, in Corollary 3.13, we show that if we remove the redundant generators in Theorem 3.11, then we obtain a result similar to [32, Theorem 4.1]. There they prove it in a slightly different setting namely $G R\left(p^{a}, m\right)$ is replaced by an arbitrary finite chain ring and $f(x)$ is either $x^{n}-1$ or $x^{n}+1$ (i.e., cyclic and negacyclic codes over a finite chain ring). We will also prove this result later in the case that $f(x)$ is an arbitrary regular polynomial.

Definition 3.12 (adapted from [27, Definition 4.1]). Let $G=\left\{p^{j_{0}} f_{j_{0}}(x), \ldots, p^{j_{r}} f_{j_{r}}(x)\right\} \subset \mathcal{R}$, for some $0 \leq r \leq a-1$, such that

(1) $0 \leq j_{0}<\cdots<j_{r} \leq a-1$,

(2) $t>k_{j_{0}}>\cdots>k_{j_{r}} \geq 0$

(3) $f_{j_{i}}(x)=h(x)^{k_{j_{i}}}+\sum_{\ell=1}^{a-1-j_{i}} p^{\ell} h(x)^{t_{j_{i}, \ell}} \alpha_{j_{i}, \ell}(x)$ where $t_{j_{i}, \ell} \operatorname{deg}(h(x))+\operatorname{deg}\left(\alpha_{j_{i}, \ell}(x)\right)<k_{j_{i}} \operatorname{deg}(h(x))$ and each $\alpha_{j_{i}, \ell}(x) \notin\langle p, h(x)\rangle \backslash\{0\}$

(4) $p^{j_{i+1}} f_{j_{i}}(x) \in\left\langle p^{j_{i+1}} f_{j_{i+1}}(x), \ldots, p^{j_{r}} f_{j_{r}}(x)\right\rangle$,

(5) $p^{j_{0}} f(x) \in\left\langle p^{j_{0}} f_{j_{0}}(x), \ldots, p^{j_{r}} f_{j_{r}}(x)\right\rangle$ in $G R\left(p^{a}, m\right)[x]$.

The set $G$ is called a generating set in standard form. Moreover, by [25, Theorem 5.4], the set $G$ is a minimal strong Groebner basis.

Corollary 3.13. Let $C \triangleleft \mathcal{R}$. There exists a generating set in standard form for $C$.

Proof. Let $\left\{f_{0}(x), \ldots, p^{a-1} f_{a-1}(x)\right\}$ be a generating set for $C$ as in Theorem 3.11. Let $j_{0}=\min \left\{i \mid f_{i}(x) \neq\right.$ $0\}$ and set $k_{i}=T_{i}(C)$. Then

$$
C=\left\langle p^{j_{0}} f_{j_{0}}(x), \ldots, p^{a-1} f_{a-1}(x)\right\rangle .
$$

Assume there exist Torsional degrees of $C, T_{i}, T_{i+1}$, such that $T_{i}=T_{i+1}$ for some $i \geq j_{0}$. It should be clear that $p^{i+1} f_{i+1}(x) \in\left\langle p^{i} f_{i}(x), p^{i+2} f_{i+2}(x), \ldots, p^{a-1} f_{a-1}(x)\right\rangle$. So after removing these unnecessary generators we have, for some $r$ such that $1 \leq r \leq a-1$,

$$
C=\left\langle p^{j_{0}} f_{j_{0}}(x), \ldots, p^{j_{r}} f_{j_{r}}(x)\right\rangle \text {. }
$$


Then the properties (11)-(4) of Definition 3.12 are satisfied.

Now, assume $p^{j_{0}} f(x) \notin\left\langle p^{j_{0}} f_{0}(x), \ldots, p^{j_{r}} f_{r}(x)\right\rangle$ in $G R\left(p^{a}, m\right)[x]$. We consider

$$
\begin{aligned}
g_{j_{0}}(x) & =p^{j_{0}} f(x)-h(x)^{t-T_{j_{0}}} p^{j_{0}} f_{j_{0}}(x) \\
& =p^{k_{0}} h(x)^{z_{k_{0}}} \alpha_{z_{k_{0}}}(x)+\cdots+p^{k_{e}} h(x)^{z_{k_{e}}} \alpha_{z_{k_{e}}}(x)
\end{aligned}
$$

where the representation (3.3) is as in (3.1). Note that $g_{j_{0}}(x) \in C$ when we consider $g_{j_{0}}(x)$ as an element of $\mathcal{R}$. If $k_{0}<j_{r}$, say $j_{q-1} \leq k_{0}<j_{q}$ for some $q \leq r$, then $z_{k_{0}} \geq T_{j_{q-1}}$ otherwise we get a contradiction to the torsional degree. Now, for an appropriate polynomial, say $v(x)$, we get

$$
\begin{aligned}
g_{j_{q-1}} & =g_{j_{0}}(x)-v(x) p^{j_{q-1}} f_{j_{q-1}}(x) \\
& =p^{\ell_{0}} h(x)^{y_{\ell_{0}}} \alpha_{y_{\ell_{0}}}(x)+\cdots+p^{\ell} e^{\prime} h(x)^{y_{\ell} e^{\prime}} \alpha_{y_{\ell^{\prime}}}(x)
\end{aligned}
$$

where the representation (3.4) is as in (3.1) and $\ell_{0}>k_{0}$. Continuing like this, we obtain a non-zero polynomial $g(x) \in\left\langle p^{j_{r}}\right\rangle$ such that

$$
p^{j_{0}} f(x)=\sum_{i=0}^{r} p^{j_{i}} f_{i}(x) \beta_{i}(x)+g(x),
$$

where $\operatorname{deg} g(x)<\operatorname{deg} f_{r}(x)$. Now, in $\mathcal{R}$

$$
g(x)=-\sum_{i=0}^{r} p^{j_{i}} f_{i}(x) \beta_{i}(x) .
$$

So, $g(x) \in C$. But, $T_{j_{r}} \operatorname{deg} h(x)>\operatorname{deg} g(x)$ which is a contradiction of the torsional degree. Hence (5) of Definition 3.12 holds.

Corollary 3.14. Let $C \triangleleft \mathcal{R}$. Then $C$ is at most $\min \{a, t\}$-generated.

Proof. Follows from the facts that the number of distinct torsional degrees that are degrees of generators in the generating set in Corollary 3.13 is less than $t$ and that the number of generators there does not exceed $a$.

Now we observe a relation between the generating sets introduced in [20, Theorem 2.5] and generating sets in standard form for cyclic codes studied in [25].

Remark 3.15. By [25, Theorem 3.2] and Corollary [3.13, a generating set as in Theorem 3.11 (and in particular, in [20, Theorem 2.5]) for $C \triangleleft \mathcal{R}$ is actually a strong Groebner basis (see [28, Definition 3.8] for a definition). Moreover, given a generating set $G$ as in Theorem 3.11, if we remove the redundant elements from $G$, as described in the proof of Corollary 3.13, we obtain a generating set as in Corollary 3.13, i.e., a generating set in standard form which is a minimal strong Groebner basis, for $C$.

Our final result of this section shows that if one can produce a generating set in standard form, the torsional degrees can easily be found.

Theorem 3.16. Let $\left\{p^{j_{0}} f_{j_{0}}(x), \ldots, p^{j_{r}} f_{j_{r}}(x)\right\}$ be a generating set in standard form for $C \triangleleft \mathcal{R}$ where $f_{j_{i}}(x)=h(x)^{k_{j_{i}}}+p \beta_{j_{i}}(x)$ for some $\beta_{j_{i}}(x) \in \mathcal{R}$. Then for $e<j_{0}, T_{e}(C)=t$; for $j_{i} \leq e<j_{i+1}$, $T_{e}(C)=k_{j_{i}}$ and for $e \geq j_{r}, T_{e}(C)=k_{j_{r}}$. 
Proof. For $e<j_{0}$, Tor $_{e}(C)=0$ so $T_{e}(C)=t$. Clearly, $T_{j_{i}}(C) \leq k_{j_{i}}$ and $T_{j_{0}}(C)=k_{j_{0}}$. Now, let $j_{i} \leq e<$ $j_{i+1}$ for some $i$. There exists a polynomial $f_{e}(x)=h(x)^{T_{e}(C)}+p \rho(x)$ where $\operatorname{deg}(\rho(x))<\operatorname{deg}(h(x)) T_{e}(C)$ such that $p^{e} f_{e}(x) \in C$. In the following we are working in $G R\left(p^{a}, m\right)[x]$. Since $e \geq j_{0}$, we have

$$
p^{e} f_{e}(x) \in\left\langle p^{j_{0}} f_{j_{0}}(x), \ldots, p^{j_{r}} f_{j_{r}}(x), p^{j_{0}} f(x)\right\rangle .
$$

By $3.12(5)$,

$$
p^{e} f_{e}(x) \in\left\langle p^{j_{0}} f_{j_{0}}(x), \ldots, p^{j_{r}} f_{j_{r}}(x)\right\rangle .
$$

We know $T_{e}(C) \leq k_{j_{i}}$. Assume $T_{e}(C)<k_{j_{i}}$. By the properties in 3.12(2) and 3.12(3), $\operatorname{deg} f_{j_{0}}(x)>\cdots>$ $\operatorname{deg} f_{j_{i}}(x)>\operatorname{deg} f_{e}(x)$ which implies

$$
p^{e} f_{e}(x) \in\left\langle p^{j_{i+1}} f_{j_{i+1}}(x), \ldots, p^{j_{r}} f_{j_{r}}(x)\right\rangle .
$$

This is a contradiction since by the property 3.12(1), $e<j_{i+1}<\cdots<j_{r} \leq a-1$ which implies

$$
p^{e} f_{e}(x) \notin\left\langle p^{j_{i+1}} f_{j_{i+1}}(x), \ldots, p^{j_{r}} f_{j_{r}}(x)\right\rangle .
$$

So, $T_{e}(C)=k_{j_{i}}$. For $e \geq j_{r}$, the proof is similar.

Remark 3.17. Remark 3.15and Theorem 3.16 imply that we can go back and forth between a generating set as in Theorem 3.11 and a generating set in standard form. Given a generating set as in Theorem 3.11 , we can obtain a generating set in standard form as explained in Remark 3.15. Conversely, suppose that we are given a generating set $G=\left\{p^{j_{0}} f_{j_{0}}(x), \ldots, p^{j_{r}} f_{j_{r}}(x)\right\}$ in standard form. We know, by Theorem 3.16 , that $f_{j_{i}}(x)=h(x)^{T_{j_{i}}}+p \beta_{j_{i}}(x)$. Define $F_{e}(x)=0$ for $0 \leq e<j_{0}, F_{e}(x)=p^{e} f_{j_{i}}(x)$ for $j_{i} \leq e<j_{i+1}$ and $F_{e}(x)=p^{j_{r}} f_{j_{r}}(x)$ for $j_{r} \leq e<a$. Then, by Theorem 3.16, the set $G^{\prime}=\left\{F_{0}(x), p F_{1}(x), \ldots, p^{a-1} F_{a-1}(x)\right\}$ is as in Theorem 3.10. Now applying the operations in the proof of Theorem 3.11 to $G^{\prime}$, we obtain a generating set as in Theorem 3.11 .

\section{Subambients in characteristic $p^{2}$}

Throughout this section, we work in characteristic $p^{2}$ and we assume $f(x) \in G R\left(p^{2}, m\right)[x]$ is a regular primary polynomial and let $\mathcal{R}_{2}=\frac{G R\left(p^{2}, m\right)[x]}{\langle f(x)\rangle}$.

Recently, the Hamming distance of cyclic codes of length $2^{s}$ over $G R(4,1)$ has been determined in [18. Applying the results of Section 3, we extend this result in two ways. First, we consider the problem for a more general class of linear codes which are called polycyclic codes. We show how to obtain the torsional degrees of polycyclic codes over a Galois ring of characteristic $p^{2}$. This gives us the Hamming distance if the Hamming distance of the residue code is known. Second, we generalize this result of [18] to cyclic codes of length $p^{s}$ over any Galois ring of characteristic $p^{2}$. We explicitly determine the Hamming distance of all cyclic codes of length $p^{s}$ over $G R\left(p^{2}, n\right)$.

First, in Lemma 4.1, we classify all polycyclic codes in characteristic $p^{s}$ where $f(x)$ is a regular primary polynomial. This also gives us a classification of all cyclic codes of length $p^{s}$. Then, in Lemma 4.2 and Lemma 4.3, we determine the torsional degrees of polycyclic codes. Using this together with some observations on the polynomial $x^{p^{s}}-1$, we determine the Hamming distance of all cyclic codes of length $p^{s}$ in characteristic $p^{2}$ in Lemma 4.8 .

As was explained in Section 3, without loss of generality, we can assume $f(x)$ is monic, $f(x)=h(x)^{t}+$ $p \beta(x)$ where $\beta(x) \in G R\left(p^{2}, m\right)[x]$ and either $\beta(x)=0$ or $\operatorname{deg} \beta(x)<t \operatorname{deg} h(x)$. Also, we may assume $h(x)$ is a monic basic irreducible polynomial. Moreover, if $\beta(x) \neq 0$ we can express $\beta(x)$ as $\beta(x)=h(x)^{v} \beta^{\prime}(x)$ such that $\beta^{\prime}(x)=\sum_{j=0}^{t-1-v} \gamma_{j}(x) h^{j}(x)$ where $v<t, \gamma_{0}(x) \neq 0, \gamma_{0}(x) \notin\langle p\rangle, \gamma_{j}(x) \in G R\left(p^{2}, m\right)[x]$ and 
$\operatorname{deg}\left(\gamma_{j}(x)\right)<\operatorname{deg}(h(x))$ (see the explanation in Section 3 ). Since we are working in characteristic $p^{2}$ we may also assume that $\gamma_{j}(x) \in \mathcal{T}_{m}[x]$. This can be seen by noting that $p \gamma_{j}(x)=p \overline{\gamma_{j}}(x)$.

Assume $C \triangleleft \mathcal{R}_{2}$. Since $C$ is finite we have that $C=\left\langle f_{1}(x), \ldots, f_{n}(x)\right\rangle$ for $f_{i}(x) \in \mathcal{R}_{2}$ where $\operatorname{deg}\left(f_{i}(x)\right)<$ $\operatorname{deg}(f(x))$, i.e. $C$ is finitely generated. Without loss of generality we can assume that if $p \nmid f_{i}(x)$ then $f_{i}(x)$ is monic and if $p \mid f_{i}(x)$ that the leading coefficient of $f_{i}(x)$ is p. We consider two cases here, when $C \nsubseteq\langle p\rangle$ and $C \subseteq\langle p\rangle$. First assume $C \nsubseteq\langle p\rangle$. In this case, it can be shown by looking at the representation (3.1) that if $p \nmid f_{i}(x)$ then $f_{i}(x)=h(x)^{k_{i}}+p h(x)^{\ell_{i}} \delta_{i}(x)$ and that if $p \mid f_{i}(x), f_{i}(x)=p h(x)^{\ell_{i}} \delta_{i}(x)$ where $\delta_{i}(x)$ is a unit with $\ell_{i} \operatorname{deg}(h(x))+\operatorname{deg}\left(\delta_{i}(x)\right)<k_{i} \operatorname{deg}(h(x))$ where at least one generator is not divisible by $p$. Let $k_{i}=\infty$ if not defined. Let $j$ be such that $k_{j}=\min \left\{k_{i}\right\}_{i=1}^{n}$. Let $g_{i}(x)=f_{i}(x)-f_{j}(x) h(x)^{k_{i}-k_{j}}$ if $p \nmid f_{i}(x)$ and $g_{i}(x)=f_{i}(x)$ if $p \mid f_{i}(x)$. Now, we see that $C=\left\langle g_{1}(x), \ldots, g_{j-1}(x), f_{j}(x), g_{j+1}(x), \ldots, g_{n}(x)\right\rangle$. Notice $g_{i}(x) \in \mathcal{R}_{2} \cap\langle p\rangle$ for $i \neq j$. Again, without loss of generality we may assume for $i \neq j$ that $g_{i}(x)=p h(x)^{\ell^{\prime}}$. Let $j^{\prime}$ be such that $\ell_{j^{\prime}}=\min \left\{\ell_{i}^{\prime}\right\}_{i=1}^{n}$. So, $g_{i}(x)-g_{j^{\prime}}(x) h(x)^{\ell_{i}^{\prime}-\ell_{j}^{\prime}}=0$. Hence, $C=\left\langle f_{j}(x), g_{j^{\prime}}(x)\right\rangle$. Finally, if $k_{j} \leq \ell_{j^{\prime}}$ then $f_{j}(x) \mid g_{j^{\prime}}(x)$ and $C=\left\langle f_{j}(x)\right\rangle$. Now, assume $C \subseteq\langle p\rangle$. Then $f_{i}(x)=p h(x)^{\ell_{i}} \delta_{i}(x)$ is a unit. Without loss of generality, we can assume $f_{i}(x)=p h(x)^{\ell_{i}}$. As above let $j$ be such that $\ell_{j}=\min \left\{\ell_{i}\right\}_{i=1}^{n}$. So, $f_{i}(x)-f_{j}(x) h(x)^{\ell_{k}-\ell_{j}}=0$. Hence, $C=\left\langle f_{j}(x)\right\rangle$. From this discussion we have the following lemma.

Lemma 4.1. Let $C \triangleleft \mathcal{R}_{2}$. Then $C$ can be expressed in one of the following forms.

(1) $\langle 0\rangle$,

(2) $\langle 1\rangle$,

(3) $\left\langle p h(x)^{n}\right\rangle$,

(4) $\left\langle h(x)^{k}\right\rangle$,

(5) $\left\langle h(x)^{k}+p h(x)^{\ell} \delta(x)\right\rangle$,

(6) $\left\langle h(x)^{k}, p h(x)^{n}\right\rangle$,

(7) $\left\langle h(x)^{k}+p h(x)^{\ell} \delta(x), p h(x)^{n}\right\rangle$

where in any case $k, \ell, n<t, \ell<n<k$ and $\delta(x)=\sum_{j=0}^{k-1-\ell} \eta_{j}(x) h(x)^{j}$ where $\eta_{j}(x) \in \mathcal{T}_{m}[x], \eta_{0}(x) \neq 0$ and $\operatorname{deg}\left(\eta_{j}(x)\right)<\operatorname{deg}(h(x))$.

Proof. The only thing that needs justification is the fact that $\delta(x)=\sum_{j=0}^{k-1-\ell} \eta_{j}(x) h(x)^{j}$ where $\eta_{j}(x) \in$ $\mathcal{T}_{m}[x], \eta_{0}(x) \neq 0$ and $\operatorname{deg}\left(\eta_{j}(x)\right)<\operatorname{deg}(h(x))$. By the discussion before this lemma, $\delta(x)$ is a unit so, $\delta(x) \notin\langle p, h(x)\rangle$. By the discussion in Section 3, $\delta(x)=\sum_{j=0}^{k-1-\ell} \eta_{j}(x) h(x)^{j}$ where $\eta_{j}(x) \in G R\left(p^{2}, m\right)[x]$, $\eta_{0}(x) \neq 0$ and $\operatorname{deg}\left(\eta_{j}(x)\right)<\operatorname{deg}(h(x))$. Finally, $\eta_{j}(x) \in \mathcal{T}_{m}[x]$ since we are working in characteristic $p^{2}$ which means $p \eta_{j}(x)=p \overline{\eta_{j}}(x)$.

The results of Section 3 assume the torsional degrees of a code are known. The next three lemmas will focus on finding the torsional degrees of a code so we can apply the results of Section 3 with the ultimate goal of this section being the determination of the Hamming distance of a code. For the following recall form the beginning of this section that $t, v, h(x), \beta(x), \beta^{\prime}(x), \gamma_{j}(x)$ are parameters of $f(x)$.

Lemma 4.2. Let $C \triangleleft \mathcal{R}_{2}$ and $n<t$. If $C=\left\langle p h(x)^{n}\right\rangle$ then $T_{0}(C)=t$ and $T_{1}(C)=n$.

Proof. The result on $T_{0}(C)$ is obvious. Since every codeword is divisible by $p$ and $h(x)^{n}$, clearly $T_{1}(C)=$ $n$.

Lemma 4.3. Assume $\beta(x)=0$. Let $C \triangleleft \mathcal{R}_{2}, k, \ell, n<t, n<k, \delta(x) \notin\langle p, h(x)\rangle$ and $\operatorname{deg}(\delta(x))<$ $(k-\ell) \operatorname{deg}(h(x))$.

(1) If $C=\left\langle h(x)^{k}\right\rangle$ then $T_{0}(C)=k$ and $T_{1}(C)=k$. 
(2) If $C=\left\langle h(x)^{k}+p h(x)^{\ell} \delta(x)\right\rangle$ then $T_{0}(C)=k$ and $T_{1}(C)=\min (k, t-k+\ell)$.

(3) If $C=\left\langle h(x)^{k}\right.$,ph $\left.(x)^{n}\right\rangle$ then $T_{0}(C)=k$ and $T_{1}(C)=\min (k, n)$.

(4) If $C=\left\langle h(x)^{k}+p h(x)^{\ell} \delta(x), p h(x)^{n}\right\rangle$ then $T_{0}(C)=k$ and $T_{1}(C)=\min (k, t-k+\ell, n)$.

Proof. The results on $T_{0}(C)$ are obvious. We concentrate on $T_{1}(C)$.

(11) The only way to create a codeword divisible by $p$ is to multiply the generator by $p$ or by a large enough power of $h(x)$. Since $h(x)^{t}=f(x)=0$ in $\mathcal{R}_{2}, h(x)^{k} h(x)^{t-k}=h(x)^{t}=f(x)=0$. Multiplying by any smaller multiple of $h(x)$ will not produce a polynomial divisible by $p$. Hence any codeword divisible by $p$ is divisible by $p h(x)^{k}$ and so $T_{1}(C)=k$.

(21) Noting that $\left(h(x)^{k}+p h(x)^{\ell} \delta(x)\right) h(x)^{t-k}=h(x)^{t}+p h(x)^{t-k+\ell} \delta(x)=p h(x)^{t-k+\ell} \delta(x)$ and $p\left(h(x)^{k}+p h(x)^{\ell} \delta(x)\right)$ $p\left(h(x)^{k}\right.$ we see that $T_{1}(C)=\min (k, t-k+\ell)$ following similar arguments as in (1).

(3) This can be argued similar to (1).

(41) This can be argued similar to (2).

Lemma 4.4. Assume $\beta(x) \neq 0$. Let $C \triangleleft \mathcal{R}_{2}, k, \ell, n<t, n<k$ and $\delta(x)=\sum_{j=0}^{k-1-\ell} \eta_{j}(x) h(x)^{j}$ where $\eta_{j}(x) \in \mathcal{T}_{m}[x], \eta_{0}(x) \neq 0$ and $\operatorname{deg}\left(\eta_{j}(x)\right)<\operatorname{deg}(h(x))$.

(1) If $C=\left\langle h(x)^{k}\right\rangle$ then $T_{0}(C)=k$ and $T_{1}(C)=\min (k, v)$.

(2) If $C=\left\langle h(x)^{k}+p h(x)^{\ell} \delta(x)\right\rangle$ then $T_{0}(C)=k$ and

$$
T_{1}(C)= \begin{cases}\min (k, v, t-k+\ell) & \text { if } v \neq t-k+\ell \\ \min (k, v+z) & \text { if } v=t-k+\ell\end{cases}
$$

where $z=\min \left(\left\{j \mid \gamma_{j}(x) \neq \eta_{j}(x)\right\} \cup\{t\}\right)$.

(3) If $C=\left\langle h(x)^{k}, p h(x)^{n}\right\rangle$ then $T_{0}(C)=k$ and $T_{1}(C)=\min (k, v, n)$.

(4) If $C=\left\langle h(x)^{k}+p h(x)^{\ell} \delta(x)\right.$, ph $\left.(x)^{n}\right\rangle$ then $T_{0}(C)=k$ and

$$
T_{1}(C)= \begin{cases}\min (k, v, t-k+\ell, n) & \text { if } v \neq t-k+\ell \\ \min (k, v+z, n) & \text { if } v=t-k+\ell\end{cases}
$$

where $z=\min \left(\left\{j \mid \gamma_{j}(x) \neq \eta_{j}(x)\right\} \cup\{t\}\right)$.

Proof. The results on $T_{0}(C)$ are obvious. We concentrate on $T_{1}(C)$.

(11) The only way to create a codeword divisible by $p$ is to multiply the generator by $p$ or by a large enough power of $h(x)$. Now, $h(x)^{t-k} h(x)^{k}=h(x)^{t}=-p h(x)^{v} \beta^{\prime}(x)$. We know $\beta^{\prime}(x)$ is a unit since $\gamma_{0}(x) \neq 0$ so, $T_{1}(C)=\min (k, v)$.

(21) First,

$$
\begin{aligned}
h(x)^{t-k}\left(h(x)^{k}+p h(x)^{\ell} \delta(x)\right) & =h(x)^{t}+p h(x)^{t-k+\ell} \delta(x) \\
& =-p h(x)^{v} \beta^{\prime}(x)+p h(x)^{t-k+\ell} \delta(x) .
\end{aligned}
$$

If $v<t-k+\ell$ then

$$
-p h(x)^{v} \beta^{\prime}(x)+p h(x)^{t-k+\ell} \delta(x)=-p h(x)^{v}\left(\gamma_{0}(x)+\sum_{j=1}^{t-1-v} \gamma_{j}(x) h^{j}(x)-h(x)^{t-k+\ell-v} \sum_{j=0}^{k-1-\ell} \eta_{j}(x) h(x)^{j}\right) .
$$

In this case $T_{1}(C)=\min (k, v)$. If $v>t-k+\ell$ then

$$
-p h(x)^{v} \beta^{\prime}(x)+p h(x)^{t-k+\ell} \delta(x)=p h(x)^{t-k+\ell}\left(\eta_{0}(x)+\sum_{j=1}^{k-1-\ell} \eta_{j}(x) h(x)^{j}-h(x)^{v-(t-k+\ell)} \sum_{j=0}^{t-1-v} \gamma_{j}(x) h^{j}(x)\right) \text {. }
$$


In this case $T_{1}(C)=\min (k, t-k+\ell)$. Next, consider the case $v=t-k+\ell$. Here, if $\beta^{\prime}(x)=\delta(x)$ then $-p h(x)^{v} \beta^{\prime}(x)+p h(x)^{t-k+\ell} \delta(x)=0$ so $T_{1}(C)=k$. Finally, if $\beta^{\prime}(x) \neq \delta(x)$ then for some $0 \leq j^{\prime}<t$, $\gamma_{j^{\prime}}(x) \neq \eta_{j^{\prime}}(x)$. Since $\gamma_{j}(x), \eta_{j}(x) \in \mathcal{T}_{m}[x]$ we have that $\gamma_{z}(x)-\eta_{z}(x)$ is not divisible by $p$ and is therefore a unit. Then

$-p h(x)^{v} \beta^{\prime}(x)+p h(x)^{t-k+\ell} \delta(x)=-p h(x)^{v+z}\left(\gamma_{z}(x)-\eta_{z}(x)+\sum_{j=z+1}^{t-1-v} \gamma_{j}(x) h^{j-z}(x)-\sum_{j=z+1}^{k-1-\ell} \eta_{j}(x) h^{j-z}(x)\right)$.

Since $z \leq t-1-v$, in this final case, $T_{1}(C)=\min (k, v+z)$.

(3) This can be argued similar to (11).

(41) This can be argued similar to (2).

Now that the torsional degrees of any code can be computed, the techniques in Section 3 can be applied to produce a generating set as in Theorem 3.11 or Definition 3.12. Our goal here is to show how the hamming distance can be computed. Notice in Section 3 that ultimately $T_{a-1}(C)$ will determine the Hamming distance of $C$, i.e., $d_{H}(C)=d_{H}\left(\overline{\left\langle h(x)^{T_{1}(C)}\right\rangle}\right)$.

In the remaining part of this section, we study cyclic codes of length $p^{s}$ over $G R\left(p^{2}, m\right)$ and show how to determine their Hamming distances. To do so we apply the results from the beginning of this section. The following two lemmas are immediate consequences of Kummer's Theorem (see [16] for the statement) which we will need for our calculations.

Lemma 4.5. Let $k<p^{e}$ and let $\ell$ be the largest integer such that $p^{\ell} \mid k$. Then $p^{e-\ell} \mid\left(\begin{array}{c}p^{e} \\ k\end{array}\right)$.

Lemma 4.6. Let $0<i<p$. We have $\left(\begin{array}{c}p^{s} \\ i p^{s-1}\end{array}\right)=p u \in G R\left(p^{2}, m\right)$ where $p \nmid u$.

To apply the results of this section, we need to show that the ambient ring is of the correct type. To do so, we only need to show that an appropriate polynomial is used for the generator of the ideal being factored out. For cyclic codes of length $p^{s}$, this polynomial is $x^{p^{s}}-1$ of course. We now show why this is an appropriate polynomial. By Lemma 4.5 and Lemma 4.6 and the fact that we are working in $G R\left(p^{2}, m\right)$,

$$
\begin{aligned}
x^{p^{s}}-1 & =((x-1)+1)^{p^{s}}-1 \\
& =(x-1)^{p^{s}}+\left(\begin{array}{c}
p^{s} \\
p^{s}-1
\end{array}\right)(x-1)^{p^{s}-1}+\cdots+\left(\begin{array}{c}
p^{s} \\
1
\end{array}\right)(x-1) \\
& =(x-1)^{p^{s}}+\left(\begin{array}{c}
p^{s} \\
(p-1) p^{s-1}
\end{array}\right)(x-1)^{(p-1) p^{s-1}}+\cdots+\left(\begin{array}{c}
p^{s} \\
p^{s-1}
\end{array}\right)(x-1)^{p^{s-1}} \\
& =(x-1)^{p^{s}}+p(x-1)^{p^{s-1}} \sum_{i=0}^{p-2} \frac{\left(\begin{array}{c}
p^{s} \\
(i+1) p^{s-1}
\end{array}\right)}{p}(x-1)^{i p^{s-1}}
\end{aligned}
$$

We want to show that we can express $x^{p^{s}}-1$ in the form needed to use the results form this section. Let $t=p^{s}, v=p^{s-1}, h(x)=x-1$ and $\beta^{\prime}(x)=\sum_{i=0}^{p-2} \gamma_{i p^{s-1}}(x-1)^{i p^{s-1}}$ where $\gamma_{i p^{s-1}}=\frac{\left(\begin{array}{c}p^{s} \\ (i+1) p^{s-1}\end{array}\right)}{p}(\bmod p)$ for $0 \leq i<p-1$ and $\gamma_{j}=0$ for all other $j$. Note, $\gamma_{j} \in \mathcal{T}_{m}$. This shows that $x^{p^{s}}-1$ is the type of polynomial we need.

The following is a special case of Lemma 4.1.

Lemma 4.7. Let $C \triangleleft \frac{G R\left(p^{2}, m\right)[x]}{\left\langle x^{p^{s}}-1\right\rangle}$. Then $C$ can be expressed in one of the following forms. 
(2) $\langle 1\rangle$,

(3) $\left\langle p(x-1)^{n}\right\rangle$,

(4) $\left\langle(x-1)^{k}\right\rangle$,

(5) $\left\langle(x-1)^{k}+p(x-1)^{\ell} \delta(x)\right\rangle$,

(6) $\left\langle(x-1)^{k}, p(x-1)^{n}\right\rangle$,

(7) $\left\langle(x-1)^{k}+p(x-1)^{\ell} \delta(x), p(x-1)^{n}\right\rangle$

where in any case $k, \ell, n<p^{s}, n<k$ and $\delta(x)=\sum_{j=0}^{k-1-\ell} \eta_{j}(x-1)^{j}$ where $\eta_{j} \in \mathcal{T}_{m}$ and $\eta_{0} \neq 0$.

Now, restating Lemma 4.2 and Lemma 4.4 for cyclic codes of length $p^{s}$ and using the fact that $d_{H}(C)=d_{H}\left(\overline{\left\langle(x-1)^{T_{1}(C)}\right\rangle}\right)$, we determine the Hamming distance of all cyclic codes of length $p^{s}$ over $G R\left(p^{2}, m\right)$ in the following lemma. Note that $\overline{\left\langle(x-1)^{T_{1}(C)}\right\rangle}$ is a cyclic code of length $p^{s}$ over $\mathbb{F}_{p^{m}}$ and its Hamming distance is given in Theorem 7.6.

Lemma 4.8. Let $C \triangleleft \frac{G R\left(p^{2}, m\right)[x]}{\left\langle x^{p^{s}}-1\right\rangle}, k, \ell, n<p^{s}, n<k$ and $\delta(x)=\sum_{j=0}^{k-1-\ell} \eta_{j}(x-1)^{j}$ where $\eta_{j} \in \mathcal{T}_{m}$ and $\eta_{0} \neq 0$. Then $d_{H}(C)=d_{H}\left(\overline{\left\langle(x-1)^{T_{1}(C)}\right\rangle}\right)$ where $T_{0}(C)$ and $T_{1}(C)$ are as follows.

(1) If $C=\left\langle(x-1)^{k}\right\rangle$ then $T_{0}(C)=k$ and $T_{1}(C)=\min \left(k, p^{s-1}\right)$.

(2) If $C=\left\langle(x-1)^{k}+p(x-1)^{\ell} \delta(x)\right\rangle$ then $T_{0}(C)=k$ and

$$
T_{1}(C)= \begin{cases}\min \left(k, p^{s-1}, p^{s}-k+\ell\right) & \text { if } p^{s-1} \neq p^{s}-k+\ell \\ \min \left(k, p^{s-1}+z\right) & \text { if } p^{s-1}=p^{s}-k+\ell\end{cases}
$$

where $z=\min \left(\left\{j \mid \gamma_{j} \neq \eta_{j}\right\} \cup\left\{p^{s}\right\}\right)$.

(3) If $C=\left\langle(x-1)^{k}, p(x-1)^{n}\right\rangle$ then $T_{0}(C)=k$ and $T_{1}(C)=\min \left(k, p^{s-1}, n\right)$.

(4) If $C=\left\langle(x-1)^{k}+p(x-1)^{\ell} \delta(x), p(x-1)^{n}\right\rangle$ then $T_{0}(C)=k$ and

$$
T_{1}(C)= \begin{cases}\min \left(k, p^{s-1}, p^{s}-k+\ell, n\right) & \text { if } p^{s-1} \neq p^{s}-k+\ell \\ \min \left(k, p^{s-1}+z, n\right) & \text { if } p^{s-1}=p^{s}-k+\ell\end{cases}
$$

where $z=\min \left(\left\{j \mid \gamma_{j} \neq \eta_{j}\right\} \cup\left\{p^{s}\right\}\right)$.

(5) If $C=\left\langle p(x-1)^{n}\right\rangle$ then $T_{0}(C)=p^{s}$ and $T_{1}(C)=n$.

\section{Structure of PolyCyClic CODE Ambients}

In this section, we study the structure of the code ambient for polycyclic codes over a Galois ring which is the ring $\frac{G R\left(p^{a}, m\right)[x]}{\langle f(x)\rangle}$ where $f(x)$ is a regular monic polynomial. Throughout this section assume that $f(x) \in G R\left(p^{a}, m\right)[x]$ is regular. By Theorem [2.2, $f(x)=\delta(x) f_{1}(x) \cdots f_{s}(x)$ where the $\delta(x) \in$ $G R\left(p^{a}, m\right)[x]$ is a unit and $\left\{f_{i}(x) \in G R\left(p^{a}, m\right)[x]\right\}_{i=1}^{s}$ is a set of regular primary co-prime polynomials that are not units. By the fact that $\delta(x)$ is a unit, we may assume without loss of generality that $f_{i}(x)=h_{i}(x)^{t_{i}}+p \beta_{i}(x)$ where $h_{i}(x)$ is a monic basic irreducible polynomial such that $\overline{h_{i}}(x)=h_{i}(x)$. We know that $t_{i} \operatorname{deg} h_{i}(x)>\operatorname{deg} \beta_{i}(x)$. Since we are interested in $\frac{G R\left(p^{a}, m\right)[x]}{\langle f(x)\rangle}$ and $\langle f(x)\rangle=\left\langle\delta(x)^{-1} f(x)\right\rangle$, we assume $\delta(x)=1$, so $f(x)=f_{1}(x) \cdots f_{s}(x)$. Additionally, throughout this section let $\mathcal{R}=\frac{G R\left(p^{a}, m\right)[x]}{\langle f(x)\rangle}$ and let $\hat{f}_{i}(x)=\prod_{j=1, j \neq i}^{s} f_{j}(x)$ for $1 \leq i \leq s$.

Theorem 5.1. For $\mathcal{R}$, we have the following

(1) $\mathcal{R}=\bigoplus_{i=1}^{s}\left\langle\hat{f}_{i}(x)+\langle f\rangle\right\rangle$ and $\left\langle\hat{f}_{i}(x)+\langle f\rangle\right\rangle \cong \frac{G R\left(p^{a}, m\right)[x]}{\left\langle f_{i}(x)\right\rangle}$,

(2) Any maximal ideal of $\mathcal{R}$ is of the form $\left\langle p \hat{f}_{i}(x)+f_{i}(x)+\langle f\rangle, h_{i} \hat{f}_{i}(x)+f_{i}(x)+\langle f\rangle\right\rangle=\left\langle p+\langle f\rangle, h_{i}(x)+\langle f\rangle\right.$ for some $1 \leq i \leq s$, 
(3) $J(\mathcal{R})=\bigcap_{i=1}^{s}\left\langle p+\langle f\rangle, h_{i}(x)+\langle f\rangle\right\rangle=\left\langle p+\langle f\rangle, \prod_{i=1}^{s} h_{i}(x)+\langle f\rangle\right\rangle$,

(4) $\operatorname{soc}(\mathcal{R})=\bigoplus_{i=1}^{s}\left\langle p^{a-1} h_{i}(x)^{t_{i}-1} \hat{f}_{i}(x)+\langle f\rangle\right\rangle=\left\langle p^{a-1} \prod_{i=1}^{s} h_{i}(x)^{t_{i}-1}+\langle f\rangle\right\rangle$.

Proof. (10) It is not hard to see that since the $f_{i}(x)$ are co-prime, $\cap\left\langle f_{i}(x)\right\rangle=\prod\left\langle f_{i}(x)\right\rangle=\langle f(x)\rangle$ (see discussion on pg. 94 in [24]). By the Chinese Remainder Theorem,

$$
\mathcal{R} \cong \bigoplus_{i=1}^{s} \frac{G R\left(p^{a}, m\right)[x]}{\left\langle f_{i}(x)\right\rangle}
$$

Define $\phi_{i}: \mathcal{R} \rightarrow \frac{G R\left(p^{a}, m\right)[x]}{\left\langle f_{i}(x)\right\rangle}$ via $\phi_{i}: a(x)+\langle f\rangle \mapsto a(x)+\left\langle f_{i}\right\rangle$. Since $\left\langle\hat{f}_{i}(x)+\langle f\rangle\right\rangle=\left\{a(x) \hat{f}_{i}(x)+\right.$ $\left.\langle f\rangle \mid \operatorname{deg} a(x)<\operatorname{deg} f_{i}(x)\right\}$ we have that $\left\langle\hat{f}_{i}(x)+\langle f\rangle\right\rangle \cong \frac{G R\left(p^{a}, m\right)[x]}{\left\langle f_{i}(x)\right\rangle}$.

(21)-(41) There exists idempotents $\hat{e}_{i}(x)+\langle f\rangle \in\left\langle\hat{f}_{i}(x)+\langle f\rangle\right\rangle$ for $1 \leq i \leq s$ such that $\left\langle\hat{e}_{i}(x)+\langle f\rangle\right\rangle=$ $\left\langle\hat{f}_{i}(x)+\langle f\rangle\right\rangle$ and $1+\langle f\rangle=\sum_{i=1}^{s} \hat{e}_{i}(x)+\langle f\rangle$. So,

$$
\begin{aligned}
\left\langle f_{j}(x)+\langle f\rangle\right\rangle & =\left\langle\left(f_{j}(x)+\langle f\rangle\right) \sum_{i=1}^{s} \hat{e}_{i}(x)+\langle f\rangle\right\rangle \\
& =\left\langle\left(f_{j}(x)+\langle f\rangle\right) \sum_{i=1, i \neq j}^{s} \hat{e}_{i}(x)+\langle f\rangle\right\rangle \\
& =\left\langle\sum_{i=1, i \neq j}^{s} \hat{e}_{i}(x)+\langle f\rangle\right\rangle \\
& =\left\langle\sum_{i=1, i \neq j}^{s} \hat{f}_{i}(x)+\langle f\rangle\right\rangle .
\end{aligned}
$$

Using (11) and Lemmas 3.1 and 3.3, the results follow.

Theorem 5.2. The following are equivalent:

(1) $\mathcal{R}$ is not a principal ideal ring.

(2) $a>1$ and there exists a factor from a primary co-prime factorization of $f(x), g(x)$, where $g(x)=h(x)^{t}+p \beta(x)$ and $h(x)$ is basic irreducible, $t>1$ and $\beta(x) \in\langle p, h(x)\rangle$.

(3) $a>1, \bar{f}(x)$ is not square free and if $\bar{f}^{\prime}(x)$ is the square free part of $\bar{f}(x)$, and we write $f(x)=$ $f^{\prime}(x) \alpha(x)+p \gamma(x)$ then $\bar{\gamma}(x)=0$ or $\bar{\alpha}(x)$ and $\bar{\gamma}(x)$ are not co-prime.

Proof. (11) $\Longleftrightarrow$ (2) By Theorem 2.2, there exists a primary coprime decomposition of $g(x)$. Then the result follows from Theorems 5.1 and 3.4 .

(2) $\Rightarrow$ (3) Since $t>1, \bar{f}(x)$ is not square free. This also shows $h(x) \mid \bar{f}^{\prime}(x)$ and $h(x) \mid \bar{\alpha}(x)$. Since $\beta(x) \in\langle p, h(x)\rangle$, we have $\bar{\beta}(x) \in\langle h\rangle$. This implies $h(x) \mid\left(g(x)\left(\bmod p^{2}\right)\right)$. Since $g(x) \mid f(x)$, we see $h(x) \mid \bar{\gamma}(x)$. So, $\bar{\alpha}(x)$ and $\bar{\gamma}(x)$ are not co-prime.

(3) $\Rightarrow$ (2) Since $\bar{f}(x)$ is not square free and $\bar{\alpha}(x)$ and $\bar{\gamma}(x)$ are not co-prime there exists a basic irreducible polynomial $h(x)$ such that $h(x)^{t} \mid \bar{f}(x)$ for some $t>1$ and $h(x) \mid \bar{\gamma}(x)$. So there exists a factor $g(x)$ of $f(x)$ such that $g(x)=h(x)^{t}+p \beta(x)$ for some $\beta(x)$. Since $h(x) \mid \bar{\gamma}(x)$, we have that $h(x) \mid \bar{\beta}(x)$. Hence, $\beta(x) \in\langle p, h(x)\rangle$.

Remark 5.3. The equivalence in Theorem 5.2 of (11) and (3) was presented in 32] with an alternative proof. 
Lemma 5.4. Let $R$ be a ring with direct sum decomposition $R=\oplus_{i=1}^{n} R_{i}$. Assume, for any positive integer $i$, that $I_{i} \triangleleft R_{i}$ is at most $k$-generated. Then $I \triangleleft R$ is at most $k$-generated.

Proof. Let $I \triangleleft R$. Then $I=\oplus_{i=1}^{n} I_{i}$ for $I_{i} \in R_{i}$. Then $I_{i}$ is generated by some $f_{i 1}, \ldots, f_{i k} \in R_{i}$. Let $g_{j}=f_{1 j}+\cdots+f_{n j}$ for $1 \leq j \leq k$. Then $\left\langle f_{1 j}, \ldots, f_{n j}\right\rangle=\left\langle g_{j}\right\rangle$ and hence $I=\left\langle g_{1}, \ldots, g_{k}\right\rangle$.

Now we generalize Proposition 3.13 to the case where $f(x)$ is an arbitrary regular polynomial.

Theorem 5.5. Let $C \triangleleft \mathcal{R}$. Then

$$
C=\left\langle p^{j_{0}} g_{0}(x), \ldots, p^{j_{r}} g_{r}(x)\right\rangle
$$

where $0 \leq r \leq a-1$ and

(1) $0 \leq j_{0}<\cdots<j_{r} \leq a-1$

(2) $g_{i}(x)$ monic for $i=0, \ldots, r$,

(3) $\operatorname{deg} f(x)>\operatorname{deg} g_{0}(x)>\cdots>\operatorname{deg} g_{r}(x)$,

(4) $p^{j_{i+1}} g_{i}(x) \in\left\langle p^{j_{i+1}} g_{i+1}(x), \ldots, p^{j_{r}} g_{r}(x)\right\rangle$

(5) $p^{j_{0}} f(x) \in\left\langle p^{j_{0}} g_{0}(x), \ldots, p^{j_{r}} g_{r}(x)\right\rangle$ in $G R\left(p^{a}, m\right)[x]$.

Proof. Follows from Proposition 3.13, Theorem 5.1 and Lemma 5.4 .

The structure of the ambient space of cyclic codes over finite chain rings was studied in [26], 28], [27] and [32]. For any ideal of the ambient space, the authors of those papers came up with a special generating set called strong Groebner basis (SGB). They showed that SGB can be used to determine the Hamming distance of the corresponding code. It is easy to see that their results also hold for the ideals of $\mathcal{R}$. So we have the following result.

Theorem 5.6. Let $C \triangleleft \mathcal{R}$ where $C=\left\langle p^{j_{0}} g_{j_{0}}(x), \ldots, p^{j_{r}} g_{j_{r}}(x)\right\rangle$ is as in Theorem 5.5. Then $d_{H}(C)=$ $d_{H}\left(\left\langle p^{a-1} g_{j_{r}}(x)\right\rangle\right)=d_{H}\left(\overline{\left\langle g_{j_{r}}(x)\right\rangle}\right)$.

Proof. For $v(x) \in C$, if $p^{k} v(x) \neq 0$ then $w_{H}(v(x)) \geq w_{H}\left(p^{k} v(x)\right)$. Let $c(x) \in C$ such that $d_{H}(I)=$ $w_{H}(c(x))$. Let $\ell$ be the largest integer such that $p^{\ell} c(x) \neq 0$. Hence, $p^{\ell} c(x) \in C \bigcap\left\langle p^{a-1}\right\rangle=\left\langle p^{a-1} g_{j_{r}}\right\rangle$. Also $w_{H}(c(x))=w_{H}\left(p^{k} c(x)\right)$ by the minimality of $c(x)$. Hence, $d_{H}\left(\left\langle p^{a-1} g_{j_{r}}(x)\right\rangle\right)=w_{H}(p c(x))=d_{H}(C)$. The equality $d_{H}\left(\left\langle p^{a-1} g_{j_{r}}(x)\right\rangle\right)=d_{H}\left(\overline{\left\langle g_{j_{r}}(x)\right\rangle}\right)$ follows from Lemma 2.3 .

\section{On the Hamming Weight of $\left(x^{n}+\gamma\right)^{N}$}

We develop some tools, that we use in Section 7 and Section 8 , to compute the Hamming distance of some constacyclic codes over finite fields.

We begin by partitioning the set $\left\{1,2, \ldots, p^{s}-1\right\}$ into three subsets. These subsets arise naturally from the technicalities of our computations as described in Section 7 and Section 8 , If $i$ is an integer satisfying $1 \leq i \leq(p-1) p^{s-1}$, then there exists a uniquely determined integer $\beta$ such that $0 \leq \beta \leq p-2$ and

$$
\beta p^{s-1}+1 \leq i \leq(\beta+1) p^{s-1}
$$

Moreover since

$$
p^{s}-p^{s-1}<p^{s}-p^{s-2}<\cdots<p^{s}-p^{s-s}=p^{s}-1,
$$


for an integer $i$ satisfying $(p-1) p^{s-1}+1=p^{s}-p^{s-1}+1 \leq i \leq p^{s}-1$, there exists a uniquely determined integer $k$ such that $1 \leq k \leq s-1$ and

$$
p^{s}-p^{s-k}+1 \leq i \leq p^{s}-p^{s-k-1} .
$$

Besides if $i$ is an integer as above and $k$ is the integer satisfying $1 \leq k \leq s-1$ and (6.1), then we have

$$
\begin{aligned}
& p^{s}-p^{s-k}<p^{s}-p^{s-k}+p^{s-k-1}<p^{s}-p^{s-k}+2 p^{s-k-1}<\cdots \\
& <p^{s}-p^{s-k}+(p-1) p^{s-k-1}
\end{aligned}
$$

and $p^{s}-p^{s-k}+(p-1) p^{s-k-1}=p^{s}-p^{s-k-1}$. So for such integers $i$ and $k$, there exists a uniquely determined integer $\tau$ with $1 \leq \tau \leq p-1$ such that

$$
p^{s}-p^{s-k}+(\tau-1) p^{s-k-1}+1 \leq i \leq p^{s}-p^{s-k}+\tau p^{s-k-1} .
$$

Thus

$$
\begin{aligned}
& \left\{1,2, \ldots, p^{s-1}\right\} \sqcup \bigsqcup_{\beta=1}^{p-2}\left\{i: \quad \beta p^{s-1}+1 \leq i \leq(\beta+1) p^{s-1}\right\} \\
& \sqcup \bigsqcup_{k=1}^{s-1} \bigsqcup_{\tau=1}^{p-1}\left\{i: \quad p^{s}-p^{s-k}+(\tau-1) p^{s-k-1}+1 \leq i \leq p^{s}-p^{s-k}+\tau p^{s-k-1}\right\}
\end{aligned}
$$

gives us a partition of the set $\left\{1,2, \ldots, p^{s}-1\right\}$.

Throughout this section $q$ denotes a power of $p$. Let $N$ be a positive integer and $\gamma \in \mathbb{F}_{q} \backslash\{0\}$. Our computations in Section 7 and Section 8 are based on expressing the Hamming weight of an arbitrary nonzero codeword in terms of $w_{H}\left(\left(x^{\eta}+\gamma\right)^{N}\right)$. In [23, the Hamming weight of the polynomial $\left(x^{\eta}+\gamma\right)^{N}$ is given as described below. Let $e, \eta, N$ and $0 \leq b_{0}, b_{1}, \ldots, b_{e-1} \leq p-1$ be positive integers such that $N<p^{e}$ and let $\gamma \in \mathbb{F}_{q} \backslash\{0\}$. Let $N=b_{e-1} p^{e-1}+\cdots+b_{1} p+b_{0}, 0 \leq b_{i}<p$, be the p-adic expansion of $N$. Then, by [23, Lemma 1], we have

$$
w_{H}\left((x+\gamma)^{N}\right)=\prod_{d=0}^{e-1}\left(b_{d}+1\right) .
$$

As suggested in [23], identifying $x$ with $x^{\eta}$ in (6.3), we obtain

$$
w_{H}\left(\left(x^{\eta}+\gamma\right)^{N}\right)=\prod_{d=0}^{e-1}\left(b_{d}+1\right) .
$$

The following two lemmas are consequences of (6.4) and we will use them in our computations frequently.

Lemma 6.1. Let $m, \eta, 1 \leq \beta \leq p-2$ be positive integers and $\gamma \in \mathbb{F}_{q} \backslash\{0\}$. If $m<p^{s}-\beta p^{s-1}-1$, then $w_{H}\left(\left(x^{\eta}+\gamma\right)^{m+\beta p^{s-1}+1}\right) \geq \beta+2$.

Proof. Since

$$
m<p^{s}-\beta p^{s-1}-1=(p-\beta-1) p^{s-1}+(p-1) p^{s-2}+\cdots+(p-1) p+p-1,
$$

either

$$
\begin{aligned}
m & =L p^{s-1}+(p-1) p^{s-2}+\cdots+(p-1) p+p-1 \text { or } \\
m & =a_{s-1} p^{s-1}+\cdots+a_{1} p+a_{0}
\end{aligned}
$$


holds, where $0 \leq L \leq p-\beta-2,0 \leq a_{0}, a_{1}, \ldots, a_{s-2} \leq p-1$ and $0 \leq a_{s-1} \leq p-\beta-1$ are integers such that $a_{\ell}<p-1$ for some $0 \leq \ell<s-1$. According to the p-adic expansion of $m$, we consider the following two cases.

First, we assume that $m=L p^{s-1}+(p-1) p^{s-2}+\cdots+(p-1) p+p-1$. Then $m+\beta p^{s-1}+1=(L+\beta+1) p^{s-1}$. So using (6.4), we get $w_{H}\left(\left(x^{\eta}+\gamma\right)^{m+\beta p^{s-1}+1}\right)=L+\beta+2 \geq \beta+2$.

Second, we assume that $m=a_{s-1} p^{s-1}+\cdots+a_{1} p+a_{0}$. Then the p-adic expansion of $m+\beta p^{s-1}+1$ is of the form $m+\beta p^{s-1}+1=b_{s-1} p^{s-1}+\cdots+b_{1} p+b_{0}$ where $0 \leq b_{0}, b_{1}, \ldots, b_{s-2} \leq p-1$ and

$$
b_{s-1}=a_{s-1}+\beta .
$$

Let $k$ be the least nonnegative integer with $a_{k}<p-1$. Then it follows that

$$
0<b_{k} \leq p-1 \text {. }
$$

So, using (6.4), (6.5) and (6.6), we get

$$
w_{H}\left(\left(x^{\eta}+\gamma\right)^{m+\beta p^{s-1}+1}\right) \geq\left(\beta+a_{s-1}+1\right)\left(b_{k}+1\right) \geq(\beta+1) 2>\beta+2 .
$$

Lemma 6.2. Let $m, \eta, 1 \leq \tau \leq p-1,1 \leq k \leq s-1$ be positive integers and $\gamma \in \mathbb{F}_{q} \backslash\{0\}$. If $m<$ $p^{s-k}-(\tau-1) p^{s-k-1}-1$, then $w_{H}\left(\left(x^{2 \eta}+\gamma\right)^{m+p^{s}-p^{s-k}+(\tau-1) p^{s-k-1}+1}\right) \geq(\tau+1) p^{k}$.

Proof. Since

$$
\begin{aligned}
m & <p^{s-k}-(\tau-1) p^{s-k-1}-1 \\
& =(p-\tau+1) p^{s-k-1}-1 \\
& =(p-\tau) p^{s-k-1}+(p-1) p^{s-k-2}+\cdots+(p-1) p+p-1,
\end{aligned}
$$

either

$$
\begin{aligned}
& m=L p^{s-k-1}+(p-1) p^{s-k-2}+\cdots+(p-1) p+p-1 \text { or } \\
& m=a_{s-k-1} p^{s-k-1}+\cdots+a_{1} p+a_{0}
\end{aligned}
$$

holds, where $0 \leq L \leq p-\tau-1,0 \leq a_{0}, a_{1}, \ldots, a_{s-k-2} \leq p-1$ and $0 \leq a_{s-k-1} \leq p-\tau$ are some integers such that $0 \leq a_{\ell}<p-1$ for some $0 \leq \ell<s-k-1$. According to the p-adic expansion of $m$, we consider the following two cases.

First, we assume that $m=L p^{s-k-1}+(p-1) p^{s-k-2}+\cdots+(p-1) p+p-1$. Then the p-adic expansion of $m+p^{s}-p^{s-k}+(\tau-1) p^{s-k-1}+1$ is of the form

$$
m+p^{s}-p^{s-k}+(\tau-1) p^{s-k-1}+1=(p-1) p^{s-1}+\cdots+(p-1) p^{s-k}+(L+\tau) p^{s-k-1} .
$$

So, using (6.4), we get $w_{H}\left(\left(x^{\eta}+\gamma\right)^{m+p^{s}-p^{s-k}+(\tau-1) p^{s-k-1}+1}\right) \geq(\tau+1) p^{k}$.

Second, we assume that $m=a_{s-k-1} p^{s-k-1}+\cdots+a_{1} p+a_{0}$. Then the p-adic expansion of $m+p^{s}-$ $p^{s-k}+(\tau-1) p^{s-k-1}+1$ is of the form

$$
\begin{aligned}
m+p^{s}-p^{s-k}+(\tau-1) p^{s-k-1}+1= & (p-1) p^{s-1}+\cdots+(p-1) p^{s-k} \\
& +b_{s-k-1} p^{s-k-1}+\cdots+b_{1} p+b_{0}
\end{aligned}
$$

where $0 \leq b_{0}, b_{1}, \ldots, b_{s-k-1} \leq p-1$ are integers. It is easy to see that

$$
b_{s-k-1}=a_{s-k-1}+\tau-1 .
$$


Let $\ell_{0}$ be the least nonnegative integer with $0 \leq a_{\ell_{0}}<p-1$. Then

$$
0<b_{\ell_{0}} \leq p-1
$$

Using (6.7), (6.8) and (6.4), we get

$$
\begin{aligned}
w_{H}\left(\left(x^{\eta}+\gamma\right)^{m+p^{s}-p^{s-k}(\tau-1) p^{s-k-1}+1}\right) & \geq p^{k}\left(b_{s-k-1}+1\right)\left(b_{\ell_{0}}+1\right) \\
& \geq 2 \tau p^{k} \\
& \geq(\tau+1) p^{k} .
\end{aligned}
$$

In [23, the authors have shown that the polynomial $\left(x^{\eta}+\gamma\right)^{N}$ has the so-called "weight retaining property" (see [23, Theorem 1.1]). As a result of this, they gave a lower bound for the Hamming weight of the polynomial $g(x)\left(x^{\eta}+\gamma\right)^{N}$ where $g(x)$ is any element of $\mathbb{F}_{q}[x]$. Let $\eta, N, \gamma$ and $g(x)$ be as above. Then, by [23, Theorem 1.3 and Theorem 6.3], the Hamming weight of $g(x)\left(x^{\eta}+\gamma\right)^{N}$ satisfies

$$
w_{H}\left(g(x)\left(x^{\eta}+\gamma\right)^{N}\right) \geq w_{H}\left(g(x) \bmod x^{\eta}+\gamma\right) \cdot w_{H}\left(\left(x^{\eta}+\gamma\right)^{N}\right) .
$$

Now we examine the Hamming weight of the polynomials $\left(x^{\eta}+\gamma_{1}\right)^{p^{s}}\left(x^{\eta}+\gamma_{2}\right)^{i}$, over $\mathbb{F}_{q}[x]$, where $0<i<p^{s}$. Let $0<i<p^{s}$ be an integer and $\gamma_{1}, \gamma_{2} \in \mathbb{F}_{q} \backslash\{0\}$. Let

$$
\left(x^{\eta}+\gamma_{2}\right)^{i}=a_{i} x^{\eta i}+a_{i-1} x^{\eta(i-1)}+\cdots+a_{0} \gamma_{2}^{i}
$$

where $a_{0}, a_{1}, \ldots, a_{i}$ are the binomial coefficients. Note that

$$
\begin{aligned}
\left(x^{\eta}+\gamma_{1}\right)^{p^{s}}\left(x^{\eta}+\gamma_{2}\right)^{i}= & \left(x^{\eta p^{s}}+\gamma_{1}^{p^{s}}\right)\left(a_{i} x^{\eta i}+a_{i-1} x^{\eta(i-1)} \gamma_{2}+\cdots+a_{0} \gamma_{2}^{i}\right) \\
= & a_{i} x^{\eta\left(i+p^{s}\right)}+a_{i-1} x^{\eta\left(i-1+p^{s}\right)} \gamma_{2}+\cdots+a_{0} x^{\eta p^{s}} \gamma_{2}^{i} \\
& +a_{i} \gamma_{1}^{p^{s}} x^{\eta i}+a_{i-1} \gamma_{1}^{p^{s}} x^{\eta(i-1)}+\cdots+a_{0} \gamma_{1}^{p^{s}} \gamma_{2}^{i} .
\end{aligned}
$$

Therefore $w_{H}\left(\left(x^{\eta}+\gamma_{1}\right)^{p^{s}}\left(x^{\eta}+\gamma_{2}\right)^{i}\right)=2 w_{H}\left(\left(x^{\eta}+\gamma_{2}\right)^{i}\right)$.

\section{Certain constacyclic codes of Length $\eta p^{s}$}

Let $\eta$ and $s$ be positive integers. Let $\gamma, \lambda \in \mathbb{F}_{p^{m}} \backslash\{0\}$ such that $\gamma^{p^{s}}=-\lambda$. All $\lambda$-cyclic codes, of length $\eta p^{s}$, over $\mathbb{F}_{p^{m}}$ correspond to the ideals of the finite ring

$$
\mathcal{R}=\frac{\mathbb{F}_{p^{m}}[x]}{\left\langle x^{\eta p^{s}}-\lambda\right\rangle} .
$$

Suppose that $x^{\eta}+\gamma$ is irreducible over $\mathbb{F}_{p^{m}}$. Then the monic divisors of $x^{\eta p^{s}}-\lambda=\left(x^{\eta}+\gamma\right)^{p^{s}}$ are exactly the elements of the set $\left\{\left(x^{\eta}+\gamma\right)^{i}: \quad 0 \leq i \leq p^{s}\right\}$. So if $x^{\eta}+\lambda$ is irreducible over $\mathbb{F}_{p^{m}}$, then the $\lambda$-cyclic codes, of length $\eta p^{s}$, over $\mathbb{F}_{p^{m}}$, are of the form $\left\langle\left(x^{\eta}+\gamma\right)^{i}\right\rangle$ where $0 \leq i \leq p^{s}$. In this section, we determine the Hamming distance of all $\lambda$-cyclic codes of length $\eta p^{s}$ over $\mathbb{F}_{p^{m}}$ and $G R\left(p^{a}, m\right)$. In Theorem [7.6, we determine the Hamming distance of $\left\langle\left(x^{\eta}+\gamma\right)^{i}\right\rangle$. As a particular case, we obtain the Hamming distance of negacyclic codes of length $2 p^{s}$ over $\mathbb{F}_{p^{m}}$ where $x^{2}+1$ is irreducible over $\mathbb{F}_{p^{m}}[x]$. Using Theorem 7.6 together with the results of Section 3 and Section [5, we determine the Hamming distance of a cyclic code of length $p^{s}$ over $G R\left(p^{a}, m\right)$.

Let $C=\left\langle\left(x^{\eta}+\gamma\right)^{i}\right\rangle$ where $0 \leq i \leq p^{s}$ is an integer and $x^{\eta}+\gamma \in \mathbb{F}_{p^{m}}[x]$ is irreducible. Obviously if $i=0$, then $C=\mathcal{R}$, i.e., $C$ is the whole space $\mathbb{F}_{p^{m}}^{\eta p^{s}}$, and if $i=p^{s}$, then $C=\{0\}$. For the remaining values of $i$, we consider the partition of the set $\left\{1,2, \ldots, p^{s}-1\right\}$ given in (6.2).

If $0<i \leq p^{s-1}$, then $d_{H}(C)$ is 2 as shown in Lemma 7.1. 
For $p^{s-1}<i<p^{s}$, we first find a lower bound on the Hamming weight of an arbitrary nonzero codeword of $C$ in Lemma 7.2 and Lemma 7.4. Next in Corollary 7.3 and Corollary 7.5, we show that there exist codewords in $C$, achieving these previously found lower bounds. This gives us the Hamming distance of $C$.

We summarize our results on $\mathcal{R}$ in Theorem [7.6. We observe that Theorem 7.6 gives the Hamming distance of negacyclic codes, of length $2 p^{s}$, over $\mathbb{F}_{p^{m}}$ where $p \equiv 3 \bmod 4$ and $m$ is an odd number. We close this section by describing how to determine the Hamming distance of certain polycyclic codes, and in particular constacyclic codes, of length $\eta p^{s}$ over $G R\left(p^{a}, m\right)$.

Lemma 7.1. Let $1 \leq i \leq p^{s-1}$ be an integer and let $C=\left\langle\left(x^{\eta}+\gamma\right)^{i}\right\rangle$. Then $d_{H}(C)=2$.

Proof. The claim follows from Lemma 2.3 and the fact that

$$
\left(x^{\eta}+\gamma\right)^{p^{s-1}-i}\left(x^{\eta}+\gamma\right)^{i}=\left(x^{\eta}+\gamma\right)^{p^{s-1}}=x^{\eta p^{s-1}}+\gamma^{p^{s-1}} \in C .
$$

Let $C=\left\langle\left(x^{\eta}+\gamma\right)^{i}\right\rangle$ for some integer $0<i<p^{s}$. For any $0 \neq c(x) \in C$, there exists a $0 \neq$ $f(x) \in \mathbb{F}_{q}[x]$ such that $c(x) \equiv f(x)\left(x^{\eta}+\gamma\right)^{i} \bmod \left(x^{\eta}+\gamma\right)^{p^{s}}$. Dividing $f(x)$ by $\left(x^{\eta}+\gamma\right)^{p^{s}-i}$, we get $f(x)=q(x)\left(x^{\eta}+\gamma\right)^{p^{s}-i}+r(x)$ where $q(x), r(x) \in \mathbb{F}_{q}[x]$ and $0 \leq \operatorname{deg}(r(x))<\eta p^{s}-\eta i$ or $r(x)=0$. We observe that

$$
\begin{aligned}
c(x) & \equiv f(x)\left(x^{\eta}+\gamma\right)^{i} \\
& \equiv\left(q(x)\left(x^{\eta}+\gamma\right)^{p^{s}-i}+r(x)\right)\left(x^{\eta}+\gamma\right)^{i} \\
& \equiv q(x)\left(x^{\eta}+\gamma\right)^{p^{s}}+r(x)\left(x^{\eta}+\gamma\right)^{i} \\
& \equiv r(x)\left(x^{\eta}+\gamma\right)^{i} \bmod \left(x^{\eta}+\gamma\right)^{p^{s}} .
\end{aligned}
$$

Consequently, for any $0 \neq c(x) \in C$, there exists $0 \neq r(x) \in \mathbb{F}_{p^{m}}[x]$ with $\operatorname{deg}(r(x))<\eta p^{s}-\eta i$ such that $c(x)=r(x)\left(x^{\eta}+\gamma\right)^{i}$, where we consider this equality in $\mathbb{F}_{p^{m}}[x]$. Therefore the Hamming weight of $c \in C$ is equal to the nonzero coefficients of $r(x)\left(x^{\eta}+\gamma\right)^{i} \in \mathbb{F}_{q}[x]$, i.e., $w_{H}(c)=w_{H}\left(r(x)\left(x^{\eta}+\gamma\right)^{i}\right)$.

In the following lemma, we give a lower bound on $d_{H}(C)$ when $p^{s-1}<i$.

Lemma 7.2. Let $1 \leq \beta \leq p-2$ be an integer and let $C=\left\langle\left(x^{\eta}+\gamma\right)^{\beta p^{s-1}+1}\right\rangle$. Then $d_{H}(C) \geq \beta+2$.

Proof. Let $0 \neq c(x) \in C$, then there exists $0 \neq f(x) \in \mathbb{F}_{q}[x]$ such that

$$
c(x) \equiv f(x)\left(x^{\eta}+\gamma\right)^{\beta p^{s-1}+1} \bmod \left(x^{\eta}+\gamma\right)^{p^{s}} .
$$

We may assume that $\operatorname{deg}(f(x))<\eta p^{s}-\eta \beta p^{s-1}-\eta=(p-\beta) \eta p^{s-1}-\eta$. We choose $m$ to be the largest nonnegative integer with $\left(x^{\eta}+\gamma\right)^{m} \mid f(x)$. Clearly $\operatorname{deg}(f(x))<(p-\beta) \eta p^{s-1}-\eta$ implies $m<(p-\beta) p^{s-1}-1$. So, by Lemma 6.1, we get

$$
w_{H}\left(\left(x^{\eta}+\gamma\right)^{m+\beta p^{s-1}+1}\right) \geq \beta+2 .
$$

For $f(x)=g(x)\left(x^{\eta}+\gamma\right)^{m}$, we have $g(x) \bmod x^{\eta}+\gamma \neq 0$ by our choice of $m$, so

$$
w_{H}\left(g(x) \bmod \left(x^{\eta}+\gamma\right)\right)>0 .
$$

Now using (7.1), (7.2) and (6.9), we obtain

$$
\begin{aligned}
w_{H}(c(x)) & =w_{H}\left(g(x)\left(x^{\eta}+\gamma\right)^{m+\beta p^{s-1}+1}\right) \\
& \geq w_{H}\left(g(x) \bmod \left(x^{\eta}+\gamma\right)\right) w_{H}\left(\left(x^{\eta}+\gamma\right)^{m}\right) \\
& \geq \beta+2 .
\end{aligned}
$$


Next we show that the lower bound given in Lemma 7.2 is achieved when $p^{s-1}<i \leq(p-1) p^{s-1}$ and this gives us the exact value of $d_{H}(C)$.

Corollary 7.3. Let $1 \leq \beta \leq p-2, \beta p^{s-1}+1 \leq i \leq(\beta+1) p^{s-1}$ be integers and let $C=\left\langle\left(x^{\eta}+\gamma\right)^{i}\right\rangle$. Then $d_{H}(C)=\beta+2$.

Proof. Lemma 7.2 and $C \subset\left\langle\left(x^{\eta}+\gamma\right)^{\beta p^{s-1}+1}\right\rangle$ imply $d_{H}(C) \geq \beta+2$. We know, by (6.4), that $w_{H}\left(\left(x^{\eta}+\right.\right.$ $\left.\gamma)^{(\beta+1) p^{s-1}}\right)=\beta+2$. Clearly $\left(x^{\eta}+\gamma\right)^{(\beta+1) p^{s-1}} \in C$ as $(\beta+1) p^{s-1} \geq i$. Thus $d_{H}(C) \leq \beta+2$. Hence $d_{H}(C)=\beta+2$.

Having covered the range $p^{s-1}<i \leq(p-1) p^{s-1}$, now we give a lower bound on $d_{H}(C)$ when $(p-1) p^{s-1}<i<p^{s}$ in the following lemma.

Lemma 7.4. Let $1 \leq \tau \leq p-1,1 \leq k \leq s-1$ be integers and let $C=\left\langle\left(x^{\eta}+\gamma\right)^{p^{s}-p^{s-k}+(\tau-1) p^{s-k-1}+1}\right\rangle$. Then $d_{H}(C) \geq(\tau+1) p^{k}$.

Proof. Let $0 \neq c(x) \in C$, then there is $0 \neq f(x) \in \mathbb{F}_{p^{m}}[x]$ such that

$$
c(x) \equiv f(x)\left(x^{\eta}+\gamma\right)^{p^{s}-p^{s-k}+(\tau-1) p^{s-k-1}+1} \bmod \left(x^{\eta}+\gamma\right)^{p^{s}} .
$$

We may assume that

$$
\operatorname{deg}(f(x))<\eta p^{s-k}-\eta(\tau-1) p^{s-k-1}-\eta .
$$

Let $m$ be the largest nonnegative integer with $\left(x^{\eta}+\gamma\right)^{m} \mid f(x)$. Then there exists $g(x) \in \mathbb{F}_{p^{m}}[x]$ such that $f(x)=g(x)\left(x^{\eta}+\gamma\right)^{m}$. By (7.3), we have $m<p^{s-k}-(\tau-1) p^{s-k-1}-1$. So, by Lemma 6.2, we get

$$
w_{H}\left(\left(x^{\eta}+\gamma\right)^{m+p^{s}-p^{s-k}+(\tau-1) p^{s-k-1}+1}\right) \geq p^{k}(\tau+1) .
$$

The maximality of $m$ implies $x^{\eta}+\gamma \nmid g(x)$ and therefore $g(x) \bmod x^{\eta}+\gamma \neq 0$. So we have

$$
w_{H}\left(g(x) \bmod x^{\eta}+\gamma\right)>0 .
$$

Now using (6.9), (7.4) and (7.5), we obtain

$$
\begin{aligned}
w_{H}(c(x)) & =w_{H}\left(g(x)\left(x^{\eta}+\gamma\right)^{m+p^{s}-p^{s-k}+(\tau-1) p^{s-k-1}+1}\right) \\
& \geq w_{H}\left(g(x) \quad \bmod x^{\eta}+\gamma\right) w_{H}\left(\left(x^{\eta}+\gamma\right)^{p^{s}-p^{s-k}+(\tau-1) p^{s-k-1}+1+m}\right) \\
& \geq p^{k}(\tau+1) .
\end{aligned}
$$

This completes the proof.

For $(p-1) p^{s-1}<i<p^{s}$, we determine $d_{H}(C)$ in Corollary 7.5 where we show the existence of a codeword that achieves the lower bound given in Lemma 7.4 .

Corollary 7.5. Let $1 \leq \tau \leq p-1,1 \leq k \leq s-1$ and $i$ be integers such that

$$
p^{s}-p^{s-k}+(\tau-1) p^{s-k-1}+1 \leq i \leq p^{s}-p^{s-k}+\tau p^{s-k-1} .
$$

Let $C=\left\langle\left(x^{\eta}+\gamma\right)^{i}\right\rangle$. Then $d_{H}(C)=(\tau+1) p^{k}$.

Proof. Lemma 7.4 and $C \subset\left\langle\left(x^{\eta}+\gamma\right)^{p^{s}-p^{s-k}+(\tau-1) p^{s-k-1}+1}\right\rangle$ implies $d_{H}(C) \geq(\tau+1) p^{k}$. We know, by (6.4), that $w_{H}\left(\left(x^{\eta}+\gamma\right)^{p^{s}-p^{s-k}+\tau p^{s-k-1}}\right)=(\tau+1) p^{k}$. Clearly $\left(x^{\eta}+\gamma\right)^{p^{s}-p^{s-k}+\tau p^{s-k-1}} \in C$ as $p^{s}-p^{s-k}+\tau p^{s-k-1} \geq$ $i$. So $d_{H}(C) \leq(\tau+1) p^{k}$. Thus we have shown $d_{H}(C)=(\tau+1) p^{k}$. 
We summarize our results in the following theorem.

Theorem 7.6. Let $p$ be a prime number, $\mathbb{F}_{p^{m}}$ a finite field of characteristic $p, \gamma \in \mathbb{F}_{q} \backslash\{0\}$ and $\eta$ be a positive integer. Suppose that $x^{\eta}+\gamma \in \mathbb{F}_{q}[x]$ is irreducible. Then the $\lambda$-cyclic codes over $\mathbb{F}_{q}$, of length $\eta p^{s}$, are of the form $C[i]=\left\langle\left(x^{\eta}+\gamma\right)^{i}\right\rangle$, where $0 \leq i \leq p^{s}$ and $\lambda=-\gamma^{p^{s}}$. If $i=0$, then $C$ is the whole space $\mathbb{F}_{p^{m}}^{\eta p^{s}}$ and if $i=p^{s}$, then $C$ is the zero space $\{\mathbf{0}\}$. For the remaining values of $i$, if $p=2$, then

$$
d_{H}(C[i])= \begin{cases}1, & \text { if } i=0 \\ 2, & \text { if } 1 \leq i \leq 2^{s-1} \\ 2^{k+1}, & \text { if } 2^{s}-2^{s-k}+1 \leq i \leq 2^{s}-2^{s-k}+\tau 2^{s-k-1} \\ & \text { where } 1 \leq k \leq s-1\end{cases}
$$

if $p$ is odd, then

$$
d_{H}(C[i])= \begin{cases}2, & \text { if } 1 \leq i \leq p^{s-1} \\ \beta+2, & \text { if } \beta p^{s-1}+1 \leq i \leq(\beta+1) p^{s-1} \text { where } 1 \leq \beta \leq p-2, \\ (\tau+1) p^{k}, & \text { if } p^{s}-p^{s-k}+(\tau-1) p^{s-k-1}+1 \leq i \leq p^{s}-p^{s-k}+\tau p^{s-k-1} \\ & \text { where } 1 \leq \tau \leq p-1 \text { and } 1 \leq k \leq s-1 .\end{cases}
$$

Remark 7.7. If we replace $\eta$ with 1 and $\gamma$ with -1 in Theorem 7.6, then we obtain the main results of [10] and [29]. Namely, we obtain [10, Theorem 4.11] and [29, Theorem 3.4].

Theorem 7.6 is still useful when the polynomial $x^{\eta}+\gamma$ is reducible over the alphabet $\mathbb{F}_{p^{m}}$.

Remark 7.8. Note that $\left\langle\left(x^{\eta}+\gamma\right)^{i}\right\rangle, 0 \leq i \leq p^{s}$ are ideals of $\mathcal{R}$ independent of the fact that $x^{\eta}+\gamma$ is irreducible. So our results from Lemma 7.1 to Corollary 7.5 hold even when the polynomial $x^{\eta}+\gamma$ is reducible over $\mathbb{F}_{p^{m}}$. But then, the cases considered above do not cover all the $\lambda$-cyclic codes of length $p^{s}$. In other words, if $x^{\eta}+\gamma$ is reducible, then there are $\lambda$-cyclic codes other than $\left\langle\left(x^{\eta}+\gamma\right)^{i}\right\rangle, 0 \leq i \leq p^{s}$ and their Hamming distance is not determined here.

Now we will apply Theorem 7.6 to a particular case. Namely, we will consider the negacyclic codes over $\mathbb{F}_{p^{m}}$ of length $2 p^{s}$ where $p$ is an odd prime. In order to apply Theorem [7.6, the polynomial $x^{2}+1$ must be irreducible over $\mathbb{F}_{p^{m}}$. A complete irreducibility criterion for $x^{2}+1$ is given in the following lemma.

Lemma 7.9. Let $p$ be an odd prime and $m$ be a positive integer. The polynomial $x^{2}+1 \in \mathbb{F}_{p^{m}}[x]$ is irreducible if and only if $p=4 k+3$ for some $k \in \mathbb{N}$ and $m$ is odd.

Proof. Follows from the order of the multiplicative group of $\mathbb{F}_{p^{m}}$.

Let $C$ be a negacyclic code of length $2 p^{s}$ over $\mathbb{F}_{p^{m}}$. If $x^{2}+1$ is irreducible over $\mathbb{F}_{p^{m}}$, then the Hamming distance of $C$ is given in the following theorem.

Theorem 7.10. Let $p=4 k+3$ be a prime for some $k \in \mathbb{N}$ and let $m \in \mathbb{N}$ be an odd number. Then the negacyclic codes over $\mathbb{F}_{p^{m}}$, of length $2 p^{s}$, are of the form $C[i]=\left\langle\left(x^{2}+1\right)^{i}\right\rangle$, where $0 \leq i \leq p^{s}$, and

$$
d_{H}(C[i])= \begin{cases}2, & \text { if } 1 \leq i \leq p^{s-1}, \\ \beta+2, & \text { if } \beta p^{s-1}+1 \leq i \leq(\beta+1) p^{s-1} \text { where } 1 \leq \beta \leq p-2, \\ (\tau+1) p^{k}, & \text { if } p^{s}-p^{s-k}+(\tau-1) p^{s-k-1}+1 \leq i \leq p^{s}-p^{s-k}+\tau p^{s-k-1} \\ & \text { where } 1 \leq \tau \leq p-1 \text { and } 1 \leq k \leq s-1 .\end{cases}
$$


For the other values of $p$ and $m, x^{2}+1$ is reducible over $\mathbb{F}_{p^{m}}$ and in this case, we determine the minimum Hamming distance of $C$ in Section 8 .

Now we describe how to determine the Hamming distance of certain polycyclic codes of length $\eta p^{s}$ over $G R\left(p^{a}, m\right)$ and, in particular, this gives us the Hamming distance of certain constacyclic codes of length $\eta p^{s}$. Let $\gamma_{0}, \lambda_{0} \in G R\left(p^{a}, m\right)$ be units such that $\bar{\gamma}_{0}=\gamma, \bar{\lambda}_{0}=\lambda$ and $\gamma_{0}^{p^{s}}=-\lambda_{0}$. According to our assumption in the beginning of this section, we have that $x^{\eta}+\bar{\gamma}_{0}$ is irreducible.

Let $f(x)=\left(x^{\eta}+\gamma_{0}\right)^{p^{s}}+p \beta(x) \in G R\left(p^{a}, m\right)[x]$ with $\operatorname{deg}(\beta(x))<\eta p^{s}$. Note that $f(x)$ in this form is a primary regular polynomial so the techniques of Section 3 can be applied.

Let $\mathcal{R}_{0}=\frac{G R\left(p^{a}, m\right)[x]}{\langle f(x)\rangle}$. Let $C=\left\langle p^{j_{0}} g_{0}(x), \ldots, p^{j_{r}} g_{r}(x)\right\rangle \triangleleft \mathcal{R}_{0}$ where the generators are as in Theorem 5.5. As was done in (3.1), we can express $g_{r}(x)$ in the canonical form

$$
g_{r}(x)=p^{0}\left(x^{\eta}+\gamma_{0}\right)^{e_{0}} \alpha_{0}(x)+\cdots+p^{a-1}\left(x^{\eta}+\gamma_{0}\right)^{e_{a-1}} \alpha_{a-1}(x)
$$

where each $\alpha_{i}(x)$ is either a unit or 0 . For $0 \neq g_{r}(x)$, we have $\alpha_{0}(x) \neq 0$ since $p \nmid g_{r}(x)$. Therefore $\alpha_{0}(x)$ is a unit. So, by Theorem [5.6, we deduce that $d_{H}(C)=d_{H}\left(\overline{\left\langle g_{r}(x)\right\rangle}\right)=d_{H}\left(\overline{\left\langle\left(x^{\eta}+\gamma\right)^{e}\right\rangle}\right)$. Now $d_{H}\left(\overline{\left\langle\left(x^{\eta}+\gamma\right)^{e_{0}}\right\rangle}\right)$ can be determined using Theorem 7.6 .

Remark 7.11. Let $\gamma, \gamma_{0}, \lambda, \lambda_{0}$ be as above. The $\lambda_{0}$-cyclic codes of length $\eta p^{s}$ over $G R\left(p^{a}, m\right)$ are the ideals of the ring $\frac{G R\left(p^{a}, m\right)[x]}{\left\langle x^{\eta p^{s}}-\lambda_{0}\right\rangle}$. Since $x^{\eta p^{s}}-\lambda_{0}=\left(x^{\eta}+\gamma_{0}\right)^{p^{s}}+p \beta^{\prime}(x)$, for some $\beta(x) \in G R\left(p^{a}, m\right)[x]$ with $\operatorname{deg}\left(\beta^{\prime}(x)\right)<\eta p^{s}$, we can determine the Hamming distance of the $\lambda_{0}$-cyclic codes of length $\eta p^{s}$ over $G R\left(p^{a}, m\right)$ as described above.

\section{Certain constacyclic codes of length $2 \eta p^{s}$}

We assume that $p$ is an odd prime number, $\eta$ and $s$ are positive integers, $\mathbb{F}_{p^{m}}$ is a finite field of characteristic $p$ and $\lambda, \xi, \psi \in \mathbb{F}_{p^{m}} \backslash\{0\}$ throughout this section.

Suppose that $\psi^{p^{s}}=\lambda$ and $x^{2 \eta}-\psi$ factors into two irreducible polynomials over $\mathbb{F}_{p^{m}}$ as

$$
x^{2 \eta}-\psi=\left(x^{\eta}-\xi\right)\left(x^{\eta}+\xi\right) .
$$

In this section, we compute the Hamming distance of $\lambda$-cyclic codes, of length $2 \eta p^{s}$, over $\mathbb{F}_{p^{m}}$ where (8.1) is satisfied. Next, we determine the Hamming distance of certain polycyclic codes, and in particular certain constacyclic codes, of length $\eta p^{s}$ over $G R\left(p^{a}, m\right)$. We know that $\lambda$-cyclic codes of length $2 \eta p^{s}$ over $\mathbb{F}_{p^{m}}$ correspond to the ideals of the finite ring

$$
\mathcal{R}=\frac{\mathbb{F}_{p^{m}}[x]}{\left\langle x^{2 \eta p^{s}}-\lambda\right\rangle}
$$

Note that, by Proposition 5.1, we have $\mathcal{R}=\left\langle x^{\eta p^{s}}+\xi^{p^{s}}\right\rangle \oplus\left\langle x^{\eta p^{s}}-\xi^{p^{s}}\right\rangle$ and $\left\langle x^{\eta p^{s}}+\xi^{p^{s}}\right\rangle \cong \frac{\mathbb{F}_{p} m[x]}{\left\langle x^{\eta p^{s}}-\xi^{p^{s}}\right\rangle}$, $\left\langle x^{\eta p^{s}}-\xi^{p^{s}}\right\rangle \cong \frac{\mathbb{F}_{p} m[x]}{\left\langle x^{\eta p^{s}}+\xi^{p^{s}}\right\rangle}$. Moreover, by Proposition [5.1, the maximal ideals of $\mathcal{R}$ are $\left\langle x^{\eta}-\xi\right\rangle$ and $\left\langle x^{\eta}+\xi\right\rangle$. Since the monic polynomials dividing $x^{2 \eta p^{s}}-\lambda$ are exactly the elements of the set $\left\{\left(x^{\eta}-\xi\right)^{i}\left(x^{\eta}+\right.\right.$ $\left.\xi)^{j}: \quad 0 \leq i, j \leq p^{s}\right\}$, the $\lambda$-cyclic codes, of length $2 \eta p^{s}$, over $\mathbb{F}_{p^{m}}$ are of the form $\left\langle\left(x^{\eta}-\xi\right)^{i}\left(x^{\eta}+\xi\right)^{j}\right\rangle$, where $0 \leq i, j \leq p^{s}$ are integers.

Let $C=\left\langle\left(x^{\eta}-\xi\right)^{i}\left(x^{\eta}+\xi\right)^{j}\right\rangle$. If $(i, j)=(0,0)$, then $C=\mathcal{R}$. If $(i, j)=\left(p^{s}, p^{s}\right)$, then $C=\{0\}$. For the remaining values of $(i, j)$, we consider the partition of the set $\left\{1,2, \ldots, p^{s}-1\right\}$ given in (6.2). 
In order to simplify and improve the presentation of our results, from Lemma 8.4 till Corollary 8.21, we consider only the cases where $i \geq j$ explicitly. We do so because the cases where $j>i$ can be treated similarly as the corresponding case of $i>j$.

Now we give an overview of the results in this section. If $i=0$, or $j=0$, or $0 \leq i, j \leq p^{s-1}$, then the Hamming distance of $C$ can easily found to be 2 as shown in Lemma 8.1 and Lemma 8.2 .

If $0<j \leq p^{s-1}$ and $p^{s-1}+1 \leq i \leq p^{s}$, then $d_{H}(C)$ is computed in Lemma 8.4. Corollary 8.5, Lemma 8.6 and Corollary 8.7

If $p^{s-1}+1 \leq j \leq i \leq(p-1) p^{s-1}$, then $d_{H}(C)$ is computed in Lemma 8.8 and Corollary 8.9 .

If $p^{s-1}+1 \leq j \leq(p-1) p^{s-1}<i \leq p^{s}-1$, then $d_{H}(C)$ is computed in Lemma 8.10 and Corollary 8.11.

If $(p-1) p^{s-1}+1 \leq j \leq i \leq p^{s}-1$, then $d_{H}(C)$ is computed in Lemma 8.12, Corollary 8.13, Lemma 8.14 and Corollary 8.15

Finally if $i=p^{s}$ and $0<j<p^{s}-1$, then $d_{H}(C)$ is computed from Lemma 8.16 till Corollary 8.21,

At the end of this section, we summarize our results in Theorem 8.22 .

We begin our computations with the case where $i=0$ or $j=0$.

Lemma 8.1. Let $0<i, j \leq p^{s}$ be integers, let $C=\left\langle\left(x^{\eta}-\xi\right)^{i}\right\rangle$ and $D=\left\langle\left(x^{\eta}+\xi\right)^{j}\right\rangle$. Then $d_{H}(C)=$ $d_{H}(D)=2$.

Proof. Since

$$
\begin{aligned}
\left(x^{\eta}-\xi\right)^{p^{s}-i}\left(x^{\eta}-\xi\right)^{i} & =x^{\eta p^{s}}-\xi^{p^{s}} \in C \text { and } \\
\left(x^{\eta}+\xi\right)^{p^{s}-j}\left(x^{\eta}+\xi\right)^{j} & =x^{\eta p^{s}}+\xi^{p^{s}} \in D
\end{aligned}
$$

we have $d_{H}(C), d_{H}(D) \leq 2$. On the other hand, $d_{H}(C), d_{H}(D) \geq 2$ by Lemma 2.3 . Hence $d_{H}(C)=$ $d_{H}(D)=2$.

Lemma 8.2. Let $C=\left\langle\left(x^{\eta}-\xi\right)^{i}\left(x^{\eta}+\xi\right)^{j}\right\rangle$, for some integers $0 \leq i, j \leq p^{s-1}$ with $(i, j) \neq(0,0)$. Then $d_{H}(C)=2$.

Proof. By Lemma 2.3, we have $d_{H}(C) \geq 2$ and

$$
\left(x^{\eta}-\xi\right)^{i}\left(x^{\eta}+\xi\right)^{j}\left(x^{\eta}-\xi\right)^{p^{s-1}-i}\left(x^{\eta}+\xi\right)^{p^{s-1}-j}=x^{2 \eta p^{s-1}}-\xi^{2 p^{s-1}} \in C
$$

implies that $d_{H}(C) \leq 2$. Hence $d_{H}(C)=2$.

Let $C=\left\langle\left(x^{\eta}-\xi\right)^{i}\left(x^{\eta}+\xi\right)^{j}\right\rangle$ for some integers $0 \leq i, j \leq p^{s}$ with $(0,0) \neq(i, j) \neq\left(p^{s}, p^{s}\right)$. Let $0 \neq c(x) \in C$, then there exists $0 \neq f(x) \in \mathbb{F}_{p^{m}}[x]$ such that $c(x) \equiv f(x)\left(x^{\eta}-\xi\right)^{i}\left(x^{\eta}+\xi\right)^{j} \bmod x^{2 \eta p^{s}}-\lambda$. Dividing $f(x)$ by $\left(x^{\eta}-\xi\right)^{p^{s}-i}\left(x^{\eta}+\xi\right)^{p^{s}-j}$, we get

$$
f(x)=q(x)\left(x^{\eta}-\xi\right)^{p^{s}-i}\left(x^{\eta}+\xi\right)^{p^{s}-j}+r(x)
$$

where $q(x), r(x) \in \mathbb{F}_{q}[x]$ and, either $r(x)=0$ or $\operatorname{deg}(r(x))<2 \eta p^{s}-\eta i-\eta j$. Since

$$
\begin{aligned}
c(x) & \equiv f(x)\left(x^{\eta}-\xi\right)^{i}\left(x^{\eta}+\xi\right)^{j} \\
& \equiv\left(q(x)\left(x^{\eta}-\xi\right)^{p^{s}-i}\left(x^{\eta}+\xi\right)^{p^{s}-j}+r(x)\right)\left(x^{\eta}-\xi\right)^{i}\left(x^{\eta}+\xi\right)^{j} \\
& \equiv q(x)\left(x^{\eta}-\xi\right)^{p^{s}}\left(x^{\eta}+\xi\right)^{p^{s}}+r(x)\left(x^{\eta}-\xi\right)^{i}\left(x^{\eta}+\xi\right)^{j} \\
& \equiv r(x)\left(x^{\eta}-\xi\right)^{i}\left(x^{\eta}+\xi\right)^{j} \bmod x^{2 \eta p^{s}}-\lambda,
\end{aligned}
$$

we may assume, without loss of generality, that $\operatorname{deg}(f(x))<2 \eta p^{s}-\eta i-\eta j$. Moreover $w_{H}\left(r(x)\left(x^{\eta}-\right.\right.$ $\left.\xi)^{i}\left(x^{\eta}+\xi\right)^{j}\right)=w_{H}(c)$ as $\operatorname{deg}\left(r(x)\left(x^{\eta}-\xi\right)^{i}\left(x^{\eta}+\xi\right)^{j}\right)<2 \eta p^{s}$. 
Let $i_{0}$ and $j_{0}$ be the largest integers with $\left(x^{\eta}-\xi\right)^{i_{0}} \mid f(x)$ and $\left(x^{\eta}+\xi\right)^{j_{0}} \mid f(x)$. Then there exists $g(x) \in \mathbb{F}_{p^{m}}[x]$ such that $f(x)=\left(x^{\eta}-\xi\right)^{i_{0}}\left(x^{\eta}+\xi\right)^{j_{0}} g(x)$ and $\left(x^{\eta}-\xi\right) \nmid g(x),\left(x^{\eta}+\xi\right) \nmid g(x)$. Clearly $\operatorname{deg}(f(x))<2 \eta p^{s}-\eta i-\eta j$ implies $i_{0}+j_{0}<2 p^{s}-i-j$. Therefore $i_{0}<p^{s}-i$ or $j_{0}<p^{s}-j$ must hold.

So if $i_{0} \geq p^{s}-i$, then $j_{0}<p^{s}-j$. For such cases, the following lemma will be used in our computations.

Lemma 8.3. Let $i, j, i_{0}, j_{0}$ be nonnegative integers such that $i \geq j, i_{0} \geq p^{s}-i$ and $j_{0}<p^{s}-j$. Let $c(x)=$ $\left(x^{\eta}-\xi\right)^{i_{0}+i}\left(x^{\eta}+\xi\right)^{j_{0}+j} g(x)$ with $x^{\eta}-\xi \nmid g(x)$ and $x^{\eta}+\xi \nmid g(x)$. Then $w_{H}(c(x)) \geq 2 w_{H}\left(\left(x^{2 \eta}-\xi^{2}\right)^{j_{0}+j}\right)$.

Proof. Since $i_{0} \geq p^{s}-i$ and $-j_{0} \geq-p^{s}+j+1$, we have $i_{0}-j_{0} \geq j-i+1$ or equivalently $i_{0}-j_{0}+i-j \geq 1$. So $c(x)=\left(x^{2 \eta}-\xi^{2}\right)^{j_{0}+j}\left(x^{\eta}-\xi\right)^{i_{0}-j_{0}+i-j} g(x)$. Dividing $\left(x^{\eta}-\xi\right)^{i_{0}-j_{0}+i-j} g(x)$ by $x^{2 \eta}-\xi^{2}$, we get

$$
\left(x^{\eta}-\xi\right)^{i_{0}-j_{0}+i-j} g(x)=\left(x^{2 \eta}-\xi^{2}\right) q(x)+r(x)
$$

for some $q(x), r(x) \in \mathbb{F}_{q}[x]$ with $r(x)=0$ or $\operatorname{deg}(r(x))<2 \eta$. Let $\theta_{1}$ and $\theta_{2}$ be any roots of $x^{\eta}-\xi$ and $x^{\eta}+\xi$, respectively, in some extension of $\mathbb{F}_{p^{m}}$. Obviously $\theta_{1}$ and $\theta_{2}$ are roots of $\left(x^{2 \eta}-\xi^{2}\right) q(x)$. First we observe that $r\left(\theta_{1}\right)=0$ as $\theta_{1}$ is a root of LHS of (8.2). Second we observe that $r\left(\theta_{2}\right) \neq 0$ as $\theta_{2}$ is not a root of LHS of (8.2). So it follows that $r(x)$ is a nonzero and nonconstant polynomial implying $w_{H}(r(x)) \geq 2$. Therefore

$$
w_{H}\left(\left(x^{\eta}-\xi\right)^{i_{0}-j_{0}+i-j} g(x) \bmod x^{2 \eta}-\xi^{2}\right)=w_{H}(r(x)) \geq 2 .
$$

Using (6.9) and (8.3), we obtain

$$
\begin{aligned}
w_{H}(c(x)) & =w_{H}\left(\left(x^{2 \eta}-\xi^{2}\right)^{j_{0}+j}\left(x^{\eta}-\xi\right)^{i_{0}-j_{0}+i-j} g(x)\right) \\
& \geq w_{H}\left(\left(x^{2 \eta}-\xi^{2}\right)^{j_{0}+j}\right) w_{H}\left(\left(x^{\eta}-\xi\right)^{i_{0}-j_{0}+i-j} g(x) \bmod x^{2 \eta}-\xi^{2}\right) \\
& \geq 2 w_{H}\left(\left(x^{2 \eta}-\xi^{2}\right)^{j_{0}+j}\right) .
\end{aligned}
$$

Now we have the machinery to obtain the Hamming distance of $C$ for the ranges $p^{s-1}<i \leq p^{s}$ and $0<j \leq p^{s}$.

In what follows, for a particular range of $i$ and $j$, we first give a lower bound on $d_{H}(C)$ in the related lemma. Then in the next corollary, we determine $d_{H}(C)$ by showing the existence of a codeword that achieves the previously found lower bound.

We compute $d_{H}(C)$ when $0<j \leq p^{s-1}<i \leq 2 p^{s-1}$ in the following lemma and corollary.

Lemma 8.4. Let $C=\left\langle\left(x^{\eta}-\xi\right)^{p^{s-1}+1}\left(x^{\eta}+\xi\right)\right\rangle$. Then $d_{H}(C) \geq 3$.

Proof. Pick $0 \neq c(x) \in C$ where $c(x) \equiv f(x)\left(x^{\eta}-\xi\right)^{p^{s-1}+1}\left(x^{\eta}+\xi\right) \bmod x^{2 \eta p^{s}}-\lambda$ for some $0 \neq f(x) \in$ $\mathbb{F}_{p^{m}}[x]$ with $\operatorname{deg}(f(x))<2 \eta p^{s}-\eta p^{s-1}-2 \eta$. Let $i_{0}$ and $j_{0}$ be the largest integers with $\left(x^{\eta}-\xi\right)^{i_{0}} \mid f(x)$ and $\left(x^{\eta}+\xi\right)^{j_{0}} \mid f(x)$. Then $f(x)$ is of the form $f(x)=\left(x^{\eta}-\xi\right)^{i_{0}}\left(x^{\eta}+\xi\right)^{j_{0}} g(x)$ for some $g(x) \in \mathbb{F}_{p^{m}}[x]$ with $x^{\eta}-\xi \nmid g(x)$ and $x^{\eta}+\xi \nmid g(x)$. Note that $i_{0}<p^{s}-p^{s-1}-1$ or $j_{0}<p^{s}-1$ holds.

If $i_{0}<p^{s}-p^{s-1}-1$, then, by Lemma 6.1.

$$
w_{H}\left(\left(x^{\eta}-\xi\right)^{i_{0}+p^{s-1}+1}\right) \geq 3 .
$$

Moreover the inequality

$$
w_{H}\left(g(x)\left(x^{\eta}+\xi\right)^{j_{0}+1} \bmod x^{\eta}-\xi\right)>0
$$

holds since $x^{\eta}-\xi \nmid g(x)$. Now using (6.9), (8.4) and (8.5), we obtain 


$$
\begin{aligned}
w_{H}(c(x)) & =w_{H}\left(f(x)\left(x^{\eta}-\xi\right)^{p^{s-1}+1}\left(x^{\eta}+\xi\right)\right) \\
& =w_{H}\left(\left(x^{\eta}-\xi\right)^{i_{0}+p^{s-1}+1}\left(x^{\eta}+\xi\right)^{j_{0}+1} g(x)\right) \\
& \geq w_{H}\left(\left(x^{\eta}-\xi\right)^{i_{0}+p^{s-1}+1}\right) w_{H}\left(\left(x^{\eta}+\xi\right)^{j_{0}+1} g(x) \quad \bmod x^{\eta}-\xi\right) \\
& \geq 3 .
\end{aligned}
$$

If $i_{0} \geq p^{s}-p^{s-1}-1$, then $j_{0}<p^{s}-1$. Clearly $w_{H}\left(\left(x^{2 \eta}-\xi^{2}\right)^{j_{0}+j}\right) \geq 2$. So, by Lemma 8.3, we have

$$
w_{H}(c(x)) \geq 2 w_{H}\left(\left(x^{2 \eta}-\xi^{2}\right)^{j_{0}+j}\right) \geq 4 .
$$

Now combining (8.6) and (8.7), we obtain $w_{H}(c(x)) \geq 3$, and hence $d_{H}(C) \geq 3$.

Corollary 8.5. Let $i, j$ be integers with $2 p^{s-1} \geq i>p^{s-1} \geq j>0$ and let $C=\left\langle\left(x^{\eta}-\xi\right)^{i}\left(x^{\eta}+\xi\right)^{j}\right\rangle$. Then $d_{H}(C)=3$.

Proof. Since $C \subset\left\langle\left(x^{\eta}-\xi\right)^{p^{s-1}+1}\left(x^{\eta}+\xi\right)\right\rangle$, we know, by Lemma 8.4 that $d_{H}(C) \geq 3$. For $\left(x^{\eta}-\xi\right)^{2 p^{s-1}}\left(x^{\eta}+\right.$ $\xi)^{2 p^{s-1}} \in C$, we have

$$
\left(x^{\eta}-\xi\right)^{2 p^{s-1}}\left(x^{\eta}+\xi\right)^{2 p^{s-1}}=\left(x^{2 \eta}-\xi^{2}\right)^{2 p^{s-1}}=x^{4 \eta p^{s-1}}-2 \xi^{2 p^{s-1}} x^{2 \eta p^{s-1}}+\xi^{4 p^{s-1}} .
$$

So $d_{H}(C) \leq 3$ and hence $d_{H}(C)=3$.

For $2 p^{s-1}<i<p^{s}$ and $0<j \leq p^{s-1}, d_{H}(C)$ is computed in the following lemma and corollary.

Lemma 8.6. Let $C=\left\langle\left(x^{\eta}-\xi\right)^{2 p^{s-1}+1}\left(x^{\eta}+\xi\right)\right\rangle$. Then $d_{H}(C) \geq 4$.

Proof. Pick $0 \neq c(x) \in C$ where $c(x) \equiv f(x)\left(x^{\eta}-\xi\right)^{2 p^{s-1}+1}\left(x^{\eta}+\xi\right) \bmod x^{2 \eta p^{s}}-\lambda$ for some $0 \neq f(x) \in$ $\mathbb{F}_{p^{m}}[x]$ with $\operatorname{deg}(f(x))<2 \eta p^{s}-2 \eta p^{s-1}-2 \eta$. Let $i_{0}$ and $j_{0}$ be the largest integers with $\left(x^{\eta}-\xi\right)^{i_{0}} \mid f(x)$ and $\left(x^{\eta}+\xi\right)^{j_{0}} \mid f(x)$. Then $f(x)$ is of the form $f(x)=\left(x^{\eta}-\xi\right)^{i_{0}}\left(x^{\eta}+\xi\right)^{j_{0}} g(x)$ for some $g(x) \in \mathbb{F}_{p^{m}}[x]$ with $x^{\eta}-\xi \nmid g(x)$ and $x^{\eta}+\xi \nmid g(x)$. Note that $i_{0}<p^{s}-2 p^{s-1}-1$ or $j_{0}<p^{s}-1$ holds since $\operatorname{deg}(f(x))<2 \eta p^{s}-2 \eta p^{s-1}-2 \eta$.

If $i_{0}<p^{s}-2 p^{s-1}-1$, then, by Lemma 6.1, we have

$$
w_{H}\left(\left(x^{\eta}-\xi\right)^{i_{0}+2 p^{s-1}+1}\right) \geq 4 .
$$

Since $x^{\eta}-\xi \nmid g(x)$,

$$
w_{H}\left(g(x)\left(x^{\eta}+\xi\right)^{j_{0}+1} \bmod x^{\eta}-\xi\right)>0
$$

holds. Now using (8.8), (8.9) and (6.9), we obtain

$$
\begin{aligned}
w_{H}(c(x)) & =w_{H}\left(f(x)\left(x^{\eta}-\xi\right)^{2 p^{s-1}+1}\left(x^{\eta}+\xi\right)\right) \\
& =w_{H}\left(\left(x^{\eta}-\xi\right)^{i_{0}+2 p^{s-1}+1}\left(x^{\eta}+\xi\right)^{j_{0}+1} g(x)\right) \\
& \geq w_{H}\left(\left(x^{\eta}+\xi\right)^{j_{0}+1} g(x) \bmod x^{\eta}-\xi\right) w_{H}\left(\left(x^{\eta}-\xi\right)^{i_{0}+2 p^{s-1}+1}\right) \\
& \geq 4 .
\end{aligned}
$$

If $i_{0} \geq p^{s}-2 p^{s-1}-1$, then $j_{0}<p^{s}-1$. Clearly $w_{H}\left(\left(x^{2 \eta}-\xi^{2}\right)^{j_{0}+1}\right) \geq 2$. So, by Lemma 8.3, we have $w_{H}(c(x)) \geq 2 w_{H}\left(\left(x^{2 \eta}-\xi^{2}\right)^{j_{0}+1}\right) \geq 4$. Hence $d_{H}(C) \geq 4$.

Corollary 8.7. Let $2 p^{s-1}<i<p^{s}$ and $0<j \leq p^{s-1}$ be integers, and let $C=\left\langle\left(x^{\eta}-\xi\right)^{i}\left(x^{\eta}+\xi\right)^{j}\right\rangle$. Then $d_{H}(C)=4$.

Proof. Since $C \subset\left\langle\left(x^{\eta}-\xi\right)^{2 p^{s-1}+1}\left(x^{\eta}+\xi\right)\right\rangle$, we know, by Lemma 8.6, that $d_{H}(C) \geq 4$. For $\left(x^{\eta}-\xi\right)^{p^{s}}\left(x^{\eta}+\right.$ $\xi)^{p^{s-1}} \in C$, we have $w_{H}\left(\left(x^{\eta}-\xi\right)^{p^{s}}\left(x^{\eta}+\xi\right)^{p^{s-1}}\right)=4$. Thus $d_{H}(C) \leq 4$ and hence $d_{H}(C)=4$. 
Next we consider the cases where $p^{s-1}<j \leq i \leq p^{s}$. We begin with computing $d_{H}(C)$ when $p^{s-1}<$ $j \leq i \leq(p-1) p^{s-1}$ in the following lemma and corollary.

Lemma 8.8. Let $1 \leq \beta^{\prime} \leq \beta \leq p-2$ be integers and $C=\left\langle\left(x^{\eta}-\xi\right)^{\beta p^{s-1}+1}\left(x^{\eta}+\xi\right)^{\beta^{\prime} p^{s-1}+1}\right\rangle$. Then $d_{H}(C) \geq \min \left\{\beta+2,2\left(\beta^{\prime}+2\right)\right\}$.

Proof. Let $0 \neq c(x) \in C$. Then there exists $0 \neq f(x) \in \mathbb{F}_{p^{m}}[x]$ such that $c(x) \equiv f(x)\left(x^{\eta}-\xi\right)^{\beta p^{s-1}+1}\left(x^{\eta}+\right.$ $\xi)^{\beta^{\prime} p^{s-1}+1} \bmod x^{2 \eta p^{s}}-\lambda$. We may assume that $\operatorname{deg}(f(x))<2 \eta p^{s}-\eta \beta p^{s-1}-\eta \beta^{\prime} p^{s-1}-2 \eta$. We consider the cases $\beta=\beta^{\prime}$ and $\beta<\beta^{\prime}$ separately.

First, we assume that $\beta=\beta^{\prime}$. Then $C=\left\langle\left(x^{\eta}-\xi\right)^{\beta p^{s-1}+1}\left(x^{\eta}+\xi\right)^{\beta^{\prime} p^{s-1}+1}\right\rangle=\left\langle\left(x^{2 \eta}-\xi^{2}\right)^{\beta p^{s-1}+1}\right\rangle$. Let $m$ be the largest nonnegative integer with $\left(x^{2 \eta}-\xi^{2}\right)^{m} \mid f(x)$. We have $m<p^{s}-\beta p^{s-1}-1$ as $\operatorname{deg}(f(x))<2 \eta p^{s}-2 \eta \beta p^{s-1}-2 \eta$. So, by Lemma 6.1, we get

$$
w_{H}\left(\left(x^{2 \eta}-\xi^{2}\right)^{\beta p^{s-1}+1+m}\right) \geq \beta+2 .
$$

Clearly $f(x)$ is of the form $f(x)=\left(x^{2 \eta}-\xi^{2}\right)^{m} g(x)$ for some $g(x) \in \mathbb{F}_{p^{m}}[x]$ where $x^{2 \eta}-\xi^{2} \nmid g(x)$. So $g(x)$ $\bmod x^{2 \eta}-\xi^{2} \neq 0$ and therefore

$$
w_{H}\left(g(x) \bmod x^{2 \eta}-\xi^{2}\right)>0 .
$$

So if $\beta=\beta^{\prime}$, then using (8.10), (8.11) and (6.9), we get

$$
\begin{aligned}
w_{H}(c(x)) & =w_{H}\left(\left(x^{2 \eta}-\xi^{2}\right)^{m+\beta p^{s-1}+1} g(x)\right) \\
& \geq w_{H}\left(g(x) \bmod x^{2 \eta}-\xi^{2}\right) w_{H}\left(\left(x^{2 \eta}-\xi^{2}\right)^{m+\beta p^{s-1}+1}\right) \\
& \geq \beta+2 .
\end{aligned}
$$

Second, we assume that $\beta^{\prime}<\beta$. For $c(x) \equiv f(x)\left(x^{\eta}-\xi\right)^{\beta p^{s-1}+1}\left(x^{\eta}+\xi\right)^{\beta^{\prime} p^{s-1}+1} \bmod x^{2 \eta p^{s}}-\lambda$, let $i_{0}$ and $j_{0}$ be the largest integers with $\left(x^{\eta}-\xi\right)^{i_{0}} \mid f(x)$ and $\left(x^{\eta}+\xi\right)^{j_{0}} \mid f(x)$. Since $\operatorname{deg}(f(x))<2 \eta p^{s}-\eta \beta p^{s-1}-$ $\eta \beta^{\prime} p^{s-1}-2 \eta$, we have $i_{0}+j_{0}<2 p^{s}-\beta p^{s-1}-\beta^{\prime} p^{s-1}-2$. Thus $i_{0}<p^{s}-\beta p^{s-1}-1$ or $j_{0}<p^{s}-\beta^{\prime} p^{s-1}-1$ holds.

If $i_{0}<p^{s}-\beta p^{s-1}-1$, then, by Lemma 6.1, we have

$$
w_{H}\left(\left(x^{\eta}-\xi\right)^{i_{0}+\beta p^{s-1}+1}\right) \geq \beta+2 .
$$

Note that $\left(x^{\eta}+\xi\right)^{j_{0}+\beta^{\prime} p^{s-1}+1} g(x) \bmod x^{\eta}-\xi \neq 0$ since $x^{\eta}-\xi \nmid\left(x^{\eta}+\xi\right)^{j_{0}+\beta^{\prime} p^{s-1}+1} g(x)$. Therefore

$$
w_{H}\left(\left(x^{\eta}+\xi\right)^{j_{0}+\beta^{\prime} p^{s-1}+1} g(x) \bmod x^{\eta}-\xi\right)>0 .
$$

Using (6.9), (8.12) and (8.13), we obtain

$$
\begin{aligned}
w_{H}(c(x)) & =w_{H}\left(\left(x^{\eta}-\xi\right)^{i_{0}+\beta p^{s-1}+1}\left(x^{\eta}+\xi\right)^{j_{0}+\beta^{\prime} p^{s-1}+1} g(x)\right) \\
& \geq w_{H}\left(\left(x^{\eta}+\xi\right)^{j_{0}+\beta^{\prime} p^{s-1}+1} g(x) \bmod x^{\eta}-\xi\right) w_{H}\left(\left(x^{\eta}-\xi\right)^{i_{0}+\beta p^{s-1}+1}\right) \\
& \geq \beta+2 .
\end{aligned}
$$

If $i_{0} \geq p^{s}-\beta p^{s-1}-1$, then $j_{0}<p^{s}-\beta^{\prime} p^{s-1}-1$. By Lemma 8.3. we get

$$
w_{H}(c(x)) \geq 2 w_{H}\left(\left(x^{2 \eta}-\xi^{2}\right)^{j_{0}+\beta^{\prime} p^{s-1}+1}\right) .
$$

For $w_{H}\left(\left(x^{2 \eta}-\xi^{2}\right)^{j_{0}+\beta^{\prime} p^{s-1}+1}\right)$, we use Lemma 6.1 and get

$$
w_{H}\left(\left(x^{2 \eta}-\xi^{2}\right)^{j_{0}+\beta^{\prime} p^{s-1}+1}\right) \geq \beta^{\prime}+2 .
$$


Combining (8.15) and (8.16), we obtain

$$
w_{H}(c(x)) \geq 2\left(\beta^{\prime}+2\right) .
$$

So if $\beta^{\prime}<\beta$, then, by (8.14) and (8.17), we get that $w_{H}(c(x)) \geq \min \left\{\beta+2,2\left(\beta^{\prime}+2\right)\right\}$. In both cases, namely $\beta=\beta^{\prime}$ and $\beta^{\prime}<\beta$, we have shown that $d_{H}(C) \geq \min \left\{\beta+2,2\left(\beta^{\prime}+2\right)\right\}$.

Corollary 8.9. Let $j \leq i, 1 \leq \beta^{\prime} \leq \beta \leq p-2$ be integers such that

$$
\begin{aligned}
\beta p^{s-1}+1 & \leq i \leq(\beta+1) p^{s-1} \quad \text { and } \\
\beta^{\prime} p^{s-1}+1 & \leq j \leq\left(\beta^{\prime}+1\right) p^{s-1} .
\end{aligned}
$$

Let $C=\left\langle\left(x^{\eta}-\xi\right)^{i}\left(x^{\eta}+\xi\right)^{j}\right\rangle$. Then $d_{H}(C)=\min \left\{\beta+2,2\left(\beta^{\prime}+2\right)\right\}$.

Proof. We know, by Lemma 8.8, that $d_{H}(C) \geq \min \left\{\beta+2,2\left(\beta^{\prime}+2\right)\right\}$. So it suffices to show $d_{H}(C) \leq$ $\min \left\{\beta+2,2\left(\beta^{\prime}+2\right)\right\}$.

First, $(\beta+1) p^{s-1} \geq i, j$ implies that $\left(x^{\eta}-\xi\right)^{(\beta+1) p^{s-1}}\left(x^{\eta}+\xi\right)^{(\beta+1) p^{s-1}}=\left(x^{2 \eta}-\xi^{2}\right)^{(\beta+1) p^{s-1}} \in C$. By (6.4), we get $w_{H}\left(\left(x^{2 \eta}-\xi^{2}\right)^{(\beta+1) p^{s-1}}\right)=\beta+2$. Therefore

$$
d_{H}(C) \leq \beta+2
$$

Second, we consider $\left(x^{\eta}-\xi\right)^{p^{s}}\left(x^{\eta}+\xi\right)^{\left(\beta^{\prime}+1\right) p^{s-1}} \in C$. Using (6.4) and the fact that $p^{s}>\left(\beta^{\prime}+1\right) p^{s-1}$, we get

$$
w_{H}\left(\left(x^{\eta}-\xi\right)^{p^{s}}\left(x^{\eta}+\xi\right)^{\left(\beta^{\prime}+1\right) p^{s-1}}\right)=2 w_{H}\left(\left(x^{\eta}+\xi\right)^{\left(\beta^{\prime}+1\right) p^{s-1}}\right)=2\left(\beta^{\prime}+2\right) .
$$

So

$$
d_{H}(C) \leq 2\left(\beta^{\prime}+2\right)
$$

Combining (8.18) and (8.19), we deduce that $d_{H}(C) \leq \min \left\{\beta+2,2\left(\beta^{\prime}+2\right)\right\}$. Therefore $d_{H}(C)=$ $\min \left\{\beta+2,2\left(\beta^{\prime}+2\right)\right\}$.

The following lemma and corollary deal with the case where $p^{s-1}<j \leq(p-1) p^{s-1}<i<p^{s}$.

Lemma 8.10. Let $1 \leq \tau \leq p-1,1 \leq \beta \leq p-2,1 \leq k \leq s-1$ be integers and $C=\left\langle\left(x^{\eta}-\right.\right.$ $\left.\xi)^{p^{s}-p^{s-k}+(\tau-1) p^{s-k-1}+1}\left(x^{\eta}+\xi\right)^{\beta p^{s-1}+1}\right\rangle$. Then $d_{H}(C) \geq 2(\beta+2)$.

Proof. Let $0 \neq c(x) \in C$. Then there exists $0 \neq f(x) \in \mathbb{F}_{q}[x]$ such that $c(x) \equiv\left(x^{\eta}-\xi\right)^{p^{s}-p^{s-k}+(\tau-1) p^{s-k-1}+1}\left(x^{\eta}+\right.$ $\xi)^{\beta p^{s-1}+1} f(x) \bmod x^{2 \eta p^{s}}-\lambda$ and $\operatorname{deg}(f(x))<\eta p^{s}+\eta p^{s-k}-\eta(\tau-1) p^{s-k-1}-\eta \beta p^{s-1}-2 \eta$. Let $i_{0}$ and $j_{0}$ be the largest integers with $\left(x^{\eta}-\xi\right)^{i_{0}} \mid f(x)$ and $\left(x^{\eta}+\xi\right)^{j_{0}} \mid f(x)$. Then $f(x)$ is of the form $f(x)=\left(x^{\eta}-\xi\right)^{i_{0}}\left(x^{\eta}+\xi\right)^{j_{0}} g(x)$ for some $g(x) \in \mathbb{F}_{p^{m}}[x]$ such that $x^{\eta}-\xi \nmid g(x)$ and $x^{\eta}+\xi \nmid g(x)$. Clearly $i_{0}+j_{0}<p^{s}+p^{s-k}-(\tau-1) p^{s-k-1}-\beta p^{s-1}-2$. So $i_{0}<p^{s-k}-(\tau-1) p^{s-k-1}-1$ or $j_{0}<p^{s}-\beta p^{s-1}-1$ holds.

If $i_{0}<p^{s-k}-(\tau-1) p^{s-k-1}-1$, then, by Lemma 8.3, we have

$$
w_{H}\left(\left(x^{\eta}-\xi\right)^{i_{0}+p^{s}-p^{s-k}+(\tau-1) p^{s-k-1}+1}\right) \geq(\tau+1) p^{k} .
$$

Since $x^{\eta}-\xi \nmid g(x)$,

$$
w_{H}\left(\left(x^{\eta}+\xi\right)^{j_{0}+\beta p^{s-1}+1} g(x) \bmod x^{\eta}-\xi\right)>0 .
$$


Using (8.20), (8.21) and (6.9), we obtain

$$
\begin{aligned}
w_{H}(c(x)) & =w_{H}\left(\left(x^{\eta}-\xi\right)^{i_{0}+p^{s}-p^{s-k}+(\tau-1) p^{s-k-1}+1}\left(x^{\eta}+\xi\right)^{j_{0}+\beta p^{s-1}+1} g(x)\right) \\
& \geq w_{H}\left(\left(x^{\eta}+\xi\right)^{j_{0}+\beta p^{s-1}+1} g(x) \bmod x^{\eta}-\xi\right) w_{H}\left(\left(x^{\eta}-\xi\right)^{i_{0}+p^{s}-p^{s-k}+(\tau-1) p^{s-k-1}+1}\right) \\
& \geq(\tau+1) p^{k} \\
& \geq 2 p \\
& \geq 2(\beta+2) .
\end{aligned}
$$

If $i_{0} \geq p^{s-k}-(\tau-1) p^{s-k-1}-1$, then $j_{0}<p^{s}-\beta p^{s-1}-1$. So, by Lemma 8.3, we get

$$
w_{H}(c(x)) \geq 2 w_{H}\left(\left(x^{2 \eta}-\xi^{2}\right)^{j_{0}+\beta p^{s-1}+1}\right) .
$$

For $w_{H}\left(\left(x^{2 \eta}-\xi^{2}\right)^{j_{0}+\beta p^{s-1}+1}\right)$, we use Lemma 6.1 and get

$$
w_{H}\left(\left(x^{2 \eta}-\xi^{2}\right)^{j_{0}+\beta p^{s-1}+1}\right)=\beta+2 .
$$

Combining (8.22) and (8.23), we obtain $w_{H}(c(x)) \geq 2(\beta+2)$. So $d_{H}(C) \geq 2(\beta+2)$.

Corollary 8.11. Let $i, j, 1 \leq \tau \leq p-1,1 \leq \beta \leq p-2$ and $1 \leq k \leq s-1$ be integers such that

$$
\begin{aligned}
p^{s}-p^{s-k}+(\tau-1) p^{s-k-1}+1 & \leq i \leq p^{s}-p^{s-k}+\tau p^{s-k-1} \\
\beta p^{s-1}+1 & \leq j \leq(\beta+1) p^{s-1}
\end{aligned}
$$

Let $C=\left\langle\left(x^{\eta}-\xi\right)^{i}\left(x^{\eta}+\xi\right)^{j}\right\rangle$. Then $d_{H}(C)=2(\beta+2)$.

Proof. Since $\left\langle\left(x^{\eta}-\xi\right) p^{p^{s}-p^{s-k}+(\tau-1) p^{s-k-1}+1}\left(x^{\eta}+\xi\right)^{\beta p^{s-1}+1}\right\rangle \supset C$, we know, by Lemma 8.10, that $d_{H}(C) \geq$ $2(\beta+2)$. So it suffices to show $d_{H}(C) \leq 2(\beta+2)$. We consider $\left(x^{\eta}-\xi\right)^{p^{s}}\left(x^{\eta}+\xi\right)^{(\beta+1) p^{s-1}} \in C$. Note that $w_{H}\left(\left(x^{\eta}-\xi\right)^{(\beta+1) p^{s-1}}\right)=\beta+2$ by (6.4). So, using the fact that $p^{s}>(\beta+1) p^{s-1}$, we obtain $w_{H}\left(\left(x^{\eta}-\xi\right)^{p^{s}}\left(x^{\eta}+\xi\right)^{(\beta+1) p^{s-1}}\right)=2(\beta+2)$. So $d_{H}(C) \leq 2(\beta+2)$, and hence $d_{H}(C)=2(\beta+2)$.

From Lemma 8.12 till Corollary 8.15, we compute $d_{H}(C)$ when $(p-1) p^{s-1}<j \leq i<p^{s}$.

Lemma 8.12. Let $1 \leq k \leq s-1,1 \leq \tau^{\prime} \leq \tau \leq p-1$,

$$
\begin{aligned}
i & =p^{s}-p^{s-k}+(\tau-1) p^{s-k-1}+1 \text { and } \\
j & =p^{s}-p^{s-k}+\left(\tau^{\prime}-1\right) p^{s-k-1}+1
\end{aligned}
$$

be integers and $C=\left\langle\left(x^{\eta}-\xi\right)^{i}\left(x^{\eta}+\xi\right)^{j}\right\rangle$. Then $d_{H}(C) \geq \min \left\{2\left(\tau^{\prime}+1\right) p^{k},(\tau+1) p^{k}\right\}$.

Proof. Let $0 \neq c(x) \in C$. Then there exists $0 \neq f(x) \in \mathbb{F}_{p^{m}}[x]$ such that $c(x) \equiv f(x)\left(x^{\eta}-\xi\right)^{i}\left(x^{\eta}+\xi\right)^{j}$ $\bmod x^{2 \eta p^{s}}-\lambda$ and $\operatorname{deg}(f(x))<2 \eta p^{s}-i \eta-j \eta$. Let $i_{0}$ and $j_{0}$ be the largest integers with $\left(x^{\eta}-\xi\right)^{i_{0}} \mid f(x)$ and $\left(x^{\eta}+\xi\right)^{j_{0}} \mid f(x)$. Then $f(x)$ is of the form $f(x)=\left(x^{\eta}-\xi\right)^{i_{0}}\left(x^{\eta}+\xi\right)^{j_{0}} g(x)$ for some $g(x) \in \mathbb{F}_{p^{m}}[x]$ with $x^{\eta}-\xi \nmid g(x)$ and $x^{\eta}+\xi \nmid g(x)$. Clearly $i_{0}+j_{0}<2 p^{s}-i-j$ and therefore $i_{0}<p^{s}-i$ or $j_{0}<p^{s}-j$ holds.

If $i_{0}<p^{s}-i$, then by Lemma 6.2 , we have

$$
w_{H}\left(\left(x^{\eta}-\xi\right)^{i_{0}+i}\right) \geq(\tau+1) p^{k} .
$$

Since $x^{\eta}-\xi \nmid g(x)$, we have $g(x)\left(x^{\eta}+\xi\right)^{j_{0}+j} \not \equiv 0 \bmod x^{\eta}-\xi$ and therefore

$$
w_{H}\left(g(x)\left(x^{\eta}+\xi\right)^{j+j_{0}} \bmod x^{\eta}-\xi\right)>0 .
$$


Using (8.24), (8.25) and (6.9), we obtain

$$
\begin{aligned}
w_{H}(c(x)) & =w_{H}\left(\left(x^{\eta}-\xi\right)^{i+i_{0}}\left(x^{\eta}+\xi\right)^{j+j_{0}} g(x)\right) \\
& \geq w_{H}\left(g(x)\left(x^{\eta}+\xi\right)^{j+j_{0}} \bmod x^{\eta}-\xi\right) w_{H}\left(\left(x^{\eta}-\xi\right)^{i+i_{0}}\right) \\
& \geq(\tau+1) p^{k} .
\end{aligned}
$$

If $i_{0} \geq p^{s}-i$, then $j_{0}<p^{s}-j$. So, by Lemma 8.3 , we have

$$
w_{H}(c(x)) \geq 2 w_{H}\left(\left(x^{2 \eta}-\xi^{2}\right)^{j_{0}+j}\right) .
$$

For $w_{H}\left(\left(x^{2 \eta}-\xi^{2}\right)^{j_{0}+j}\right)$, we use Lemma 6.2 and get

$$
w_{H}\left(\left(x^{2 \eta}-\xi^{2}\right)^{j_{0}+j}\right) \geq\left(\tau^{\prime}+1\right) p^{k} .
$$

Combining (8.27) and (8.28), we obtain

$$
w_{H}(c(x)) \geq 2\left(\tau^{\prime}+1\right) p^{k} .
$$

Now, using (8.26) and (8.29), we deduce that $w_{H}(c(x)) \geq \min \left\{2\left(\tau^{\prime}+1\right) p^{k},(\tau+1) p^{k}\right\}$. Hence $d_{H}(C) \geq$ $\min \left\{2\left(\tau^{\prime}+1\right) p^{k},(\tau+1) p^{k}\right\}$.

Corollary 8.13. Let $j \leq i, 1 \leq k \leq s-1,1 \leq \tau^{\prime} \leq \tau \leq p-1$ be integers such that

$$
\begin{aligned}
p^{s}-p^{s-k}+(\tau-1) p^{s-k-1}+1 & \leq i \leq p^{s}-p^{s-k}+\tau p^{s-k-1} \\
p^{s}-p^{s-k}+\left(\tau^{\prime}-1\right) p^{s-k-1}+1 & \leq j \leq p^{s}-p^{s-k}+\tau^{\prime} p^{s-k-1}
\end{aligned} \text { and }
$$

Let $C=\left\langle\left(x^{\eta}-\xi\right)^{i}\left(x^{\eta}+\xi\right)^{j}\right\rangle$. Then $d_{H}(C)=\min \left\{2\left(\tau^{\prime}+1\right) p^{k},(\tau+1) p^{k}\right\}$.

Proof. Since $\left\langle\left(x^{\eta}-\xi\right)^{p^{s}-p^{s-k}+(\tau-1) p^{s-k-1}+1}\left(x^{\eta}+\xi\right)^{p^{s}-p^{s-k}+\left(\tau^{\prime}-1\right) p^{s-k-1}+1}\right\rangle \supset C$, we have, by Lemma 8.12, that $d_{H}(C) \geq \min \left\{2\left(\tau^{\prime}+1\right) p^{k},(\tau+1) p^{k}\right\}$. So it suffices to show $d_{H}(C) \leq \min \left\{2\left(\tau^{\prime}+1\right) p^{k},(\tau+1) p^{k}\right\}$.

First, we consider $\left(x^{\eta}-\xi\right)^{p^{s}}\left(x^{\eta}+\xi\right)^{p^{s}-p^{s-k}+\tau^{\prime} p^{s-k-1}} \in C$. Since

$$
w_{H}\left(\left(x^{\eta}+\xi\right)^{p^{s}-p^{s-1}+\tau^{\prime} p^{s-k-1}}\right)=\left(\tau^{\prime}+1\right) p^{k},
$$

we have $w_{H}\left(\left(x^{\eta}-\xi\right)^{p^{s}}\left(x^{\eta}+\xi\right)^{p^{s}-p^{s-1}+\left(\tau^{\prime}-1\right) p^{s-k-1}}\right)=2\left(\tau^{\prime}+1\right) p^{k}$. So

$$
d_{H}(C) \leq 2\left(\tau^{\prime}+1\right) p^{k}
$$

Second, we consider $\left(x^{2 \eta}-\xi^{2}\right)^{p^{s}-p^{s-k}+(\tau-1) p^{s-k-1}+1} \in C$. By Lemma 6.4, we get

$$
w_{H}\left(\left(x^{2 \eta}-\xi^{2}\right)^{p^{s}-p^{s-k}+(\tau-1) p^{s-k-1}+1}\right)=(\tau+1) p^{k} .
$$

Thus

$$
d_{H}(C) \leq(\tau+1) p^{k} .
$$

Now combining (8.30) and (8.31), we deduce that $d_{H}(C) \leq \min \left\{2\left(\tau^{\prime}+1\right) p^{k},(\tau+1) p^{k}\right\}$. Hence $d_{H}(C)=$ $\min \left\{2\left(\tau^{\prime}+1\right) p^{k},(\tau+1) p^{k}\right\}$.

Lemma 8.14. Let $1 \leq k^{\prime}<k \leq s-1,1 \leq \tau^{\prime}, \tau<p-1$,

$$
\begin{aligned}
i & =p^{s}-p^{s-k}+(\tau-1) p^{s-k-1}+1 \quad \text { and } \\
j & =p^{s}-p^{s-k^{\prime}}+\left(\tau^{\prime}-1\right) p^{s-k^{\prime}-1}+1
\end{aligned}
$$

be integers and $C=\left\langle\left(x^{\eta}-\xi\right)^{i}\left(x^{\eta}+\xi\right)^{j}\right\rangle$. Then $d_{H}(C) \geq 2\left(\tau^{\prime}+1\right) p^{k^{\prime}}$. 
Proof. Let $0 \neq c(x) \in C$. Then there exists $0 \neq f(x) \in \mathbb{F}_{p^{m}}[x]$ such that $c(x) \equiv\left(x^{\eta}-\xi\right)^{i}\left(x^{\eta}+\xi\right)^{j} f(x)$ $\bmod x^{2 \eta p^{s}}-\lambda$ and $\operatorname{deg}(f(x))<2 \eta p^{s}-i \eta-j \eta$. Let $i_{0}$ and $j_{0}$ be the largest integers with $\left(x^{\eta}-\xi\right)^{i_{0}} \mid f(x)$ and $\left(x^{\eta}+\xi\right)^{j_{0}} \mid f(x)$. Then $f(x)$ is of the form $f(x)=\left(x^{\eta}-\xi\right)^{i_{0}}\left(x^{\eta}+\xi\right)^{j_{0}} g(x)$ for some $g(x) \in \mathbb{F}_{p^{m}}[x]$ with $x^{\eta}-\xi \nmid g(x)$ and $x^{\eta}+\xi \nmid g(x)$. Clearly $i_{0}+j_{0}<2 p^{s}-i-j$. So $i_{0}<p^{s}-i$ or $j_{0}<p^{s}-j$ holds.

If $i_{0}<p^{s}-i$, then, by Lemma 6.2, we have

$$
w_{H}\left(\left(x^{\eta}-\xi\right)^{i+i_{0}}\right) \geq(\tau+1) p^{k} \geq 2\left(\tau^{\prime}+1\right) p^{k^{\prime}} .
$$

Since $x^{\eta}-\xi \nmid g(x)$, we have $\left(x^{\eta}+\xi\right)^{j_{0}+j} g(x) \bmod x^{\eta}-\xi \neq 0$ and therefore

$$
w_{H}\left(\left(x^{\eta}+\xi\right)^{j_{0}+j} g(x) \quad \bmod x^{\eta}-\xi\right)>0 .
$$

Using (8.32), (8.33) and (6.9), we obtain

$$
\begin{aligned}
w_{H}(c(x)) & =w_{H}\left(\left(x^{\eta}-\xi\right)^{i_{0}+i}\left(x^{\eta}+\xi\right)^{j_{0}+j} g(x)\right) \\
& \geq w_{H}\left(\left(x^{\eta}+\xi\right)^{j_{0}+j} g(x) \bmod x^{\eta}-\xi\right) w_{H}\left(\left(x^{\eta}-\xi\right)^{i_{0}+i}\right) \\
& \geq 2\left(\tau^{\prime}+1\right) p^{k^{\prime}}
\end{aligned}
$$

If $i_{0} \geq p^{s}-i$, then $j_{0}<p^{s}-j$. So, by Lemma 8.3 , we have

$$
w_{H}(c(x)) \geq 2 w_{H}\left(\left(x^{2 \eta}-\xi^{2}\right)^{j_{0}+j}\right) .
$$

For $w_{H}\left(\left(x^{2 \eta}-\xi^{2}\right)^{j_{0}+j}\right)$, we use Lemma 6.2 and get

$$
w_{H}\left(\left(x^{2 \eta}-\xi^{2}\right)^{j_{0}+j}\right) \geq\left(\tau^{\prime}+1\right) p^{k^{\prime}} .
$$

Now combining (8.34) and (8.35), we obtain $w_{H}(c(x)) \geq 2\left(\tau^{\prime}+1\right) p^{k^{\prime}}$. Hence $d_{H}(C) \geq 2\left(\tau^{\prime}+1\right) p^{k^{\prime}}$.

Corollary 8.15. Let $i, j, 1 \leq k^{\prime}<k \leq s-1,1 \leq \tau^{\prime}, \tau \leq p-1$ be integers such that

$$
\begin{aligned}
p^{s}-p^{s-k}+(\tau-1) p^{s-k-1}+1 & \leq i \leq p^{s}-p^{s-k}+\tau p^{s-k-1} \quad \text { and } \\
p^{s}-p^{s-k^{\prime}}+\left(\tau^{\prime}-1\right) p^{s-k^{\prime}-1}+1 & \leq j \leq p^{s}-p^{s-k^{\prime}}+\tau^{\prime} p^{s-k^{\prime}-1} .
\end{aligned}
$$

Let $C=\left\langle\left(x^{\eta}-\xi\right)^{i}\left(x^{\eta}+\xi\right)^{j}\right\rangle$. Then $d_{H}(C)=2\left(\tau^{\prime}+1\right) p^{k^{\prime}}$.

Proof. Since $\left\langle\left(x^{\eta}-\xi\right)^{p^{s}-p^{s-k}+(\tau-1) p^{s-k-1}+1}\left(x^{\eta}+\xi\right)^{p^{s}-p^{s-k^{\prime}}+\left(\tau^{\prime}-1\right) p^{s-k^{\prime}-1}+1}\right\rangle \supset C$, we know, by Lemma 8.14, that $d_{H}(C) \geq 2\left(\tau^{\prime}+1\right) p^{k^{\prime}}$. So it suffices to show $d_{H}(C) \leq 2\left(\tau^{\prime}+1\right) p^{k^{\prime}}$. We consider $\left(x^{\eta}-\xi\right)^{p^{s}}\left(x^{\eta}+\right.$

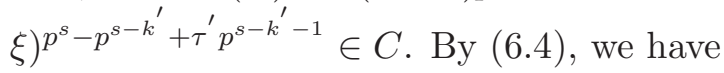

$$
w_{H}\left(\left(x^{\eta}+\xi\right)^{p^{s}-p^{s-k^{\prime}}+\tau^{\prime} p^{s-k^{\prime}-1}}\right)=\left(\tau^{\prime}+1\right) p^{k^{\prime}} .
$$

Moreover since $\left(x^{\eta}-\xi\right)^{p^{s}}=x^{\eta p^{s}}-\xi^{p^{s}}$ and $p^{s}>p^{s}-p^{s-k^{\prime}}+\tau^{\prime} p^{s-k^{\prime}-1}$, we get

$$
w_{H}\left(\left(x^{\eta}-\xi\right)^{p^{s}}\left(x^{\eta}+\xi\right)^{p^{s}-p^{s-k^{\prime}}+\tau^{\prime} p^{s-k^{\prime}-1}}\right)=2\left(\tau^{\prime}+1\right) p^{k^{\prime}} .
$$

So $d_{H}(C) \leq 2\left(\tau^{\prime}+1\right) p^{k^{\prime}}$ and therefore $d_{H}(C)=2\left(\tau^{\prime}+1\right) p^{k^{\prime}}$.

Finally it remains to consider the cases where $i=p^{s}$ and $0<j<p^{s}$.

Lemma 8.16. Let $C=\left\langle\left(x^{\eta}-\xi\right)^{p^{s}}\left(x^{\eta}+\xi\right)\right\rangle$. Then $d_{H}(C) \geq 4$. 
Proof. Pick $0 \neq c(x) \in C$. Then there exists $0 \neq f(x) \in \mathbb{F}_{p^{m}}[x]$ such that $c(x) \equiv f(x)\left(x^{\eta}-\xi\right)^{p^{s}}\left(x^{\eta}+\xi\right)$ $\bmod x^{2 \eta p^{s}}-\lambda$ and $\operatorname{deg}(f(x))<2 \eta p^{s}-\eta p^{s}-\eta=\eta p^{s}-\eta$. Let $i_{0}$ and $j_{0}$ be the largest nonnegative integers such that $\left(x^{\eta}-\xi\right)^{i_{0}} \mid f(x)$ and $\left(x^{\eta}+\xi\right)^{j_{0}} \mid f(x)$. Clearly $i_{0}+j_{0}<p^{s}-1$ as $\operatorname{deg}(f(x))<\eta p^{s}-\eta$. So, since $i_{0} \geq p^{s}-p^{s}=0$ and $j_{0}<p^{s}-1$, by Lemma 8.3, we get $w_{H}(c(x)) \geq 2 w_{H}\left(\left(x^{2 \eta}-\xi^{2}\right)^{j_{0}+1}\right)$. Obviously $w_{H}\left(\left(x^{2 \eta}-\xi^{2}\right)^{j_{0}+1}\right) \geq 2$ and therefore $w_{H}(c(x)) \geq 4$. Hence $d_{H}(C) \geq 4$.

Corollary 8.17. Let $0<j \leq p^{s-1}$ be an integer and $C=\left\langle\left(x^{\eta}-\xi\right)^{p^{s}}\left(x^{\eta}+\xi\right)^{j}\right\rangle$. Then $d_{H}(C)=4$.

Proof. Since $\left\langle\left(x^{\eta}-\xi\right)^{p^{s}}\left(x^{\eta}+\xi\right)\right\rangle \supset C$, we know, by Lemma 8.16, that $d_{H}(C) \geq 4$. So it suffices to show $d_{H}(C) \leq 4$. We consider $\left(x^{\eta}-\xi\right)^{p^{s}}\left(x^{\eta}+\xi\right)^{p^{s-1}} \in C$. Clearly $w_{H}\left(\left(x^{\eta}-\xi\right)^{p^{s}}\left(x^{\eta}+\xi\right)^{p^{s-1}}\right)=4$. So $d_{H}(C) \leq 4$ and hence $d_{H}(C)=4$.

For $i=p^{s}$ and $p^{s-1}<j<p^{s}$, the Hamming distance of $C$ is computed in the following lemmas and corollaries. Their proofs are similar to those of Lemma 8.16 and Corollary 8.16 .

Lemma 8.18. Let $1 \leq \beta \leq p-2$ be an integer and $C=\left\langle\left(x^{\eta}-\xi\right)^{p^{s}}\left(x^{\eta}+\xi\right)^{\beta p^{s-1}+1}\right\rangle$. Then $d_{H}(C) \geq$ $2(\beta+2)$.

Corollary 8.19. Let $1 \leq \beta \leq p-2, \beta p^{s-1}+1 \leq j \leq(\beta+1) p^{s-1}$ be integers. Let $C=\left\langle\left(x^{\eta}-\xi\right)^{p^{s}}\left(x^{\eta}+\xi\right)^{j}\right\rangle$. Then $d_{H}(C)=2(\beta+2)$.

Lemma 8.20. Let $1 \leq \tau \leq p-1,1 \leq k \leq s-1, j$ be integers and $C=\left\langle\left(x^{\eta}-\xi\right)^{p^{s}}\left(x^{\eta}+\xi\right)^{p^{s}-p^{s-k}+(\tau-1) p^{s-k-1}+1}\right\rangle$. Then $d_{H}(C) \geq 2(\tau+1) p^{k}$.

Corollary 8.21. Let $1 \leq \tau \leq p-1,1 \leq k \leq s-1, j$ be integers such that

$$
p^{s}-p^{s-k}+(\tau-1) p^{s-k-1}+1 \leq j \leq p^{s}-p^{s-k}+\tau p^{s-k-1} .
$$

Let $C=\left\langle\left(x^{\eta}-\xi\right)^{p^{s}}\left(x^{\eta}+\xi\right)^{j}\right\rangle$. Then $d_{H}(C)=2(\tau+1) p^{k}$.

We summarize our results in the following theorem.

Theorem 8.22. Let $p$ be an odd prime, $a, s, n$ be arbitrary positive integers. Let $\lambda, \xi, \psi \in \mathbb{F}_{p^{m}} \backslash\{0\}$ such that $\lambda=\psi^{p^{s}}$. Suppose that the polynomial $x^{2 \eta}-\psi$ factors into two irreducible polynomials as $x^{2 \eta}-\psi=$ $\left(x^{\eta}-\xi\right)\left(x^{\eta}+\xi\right)$. Then all $\lambda$-cyclic codes, of length $2 \eta p^{s}$, over $\mathbb{F}_{p^{m}}$ are of the form $\left\langle\left(x^{\eta}-\xi\right)^{i}\left(x^{\eta}+\xi\right)^{j}\right\rangle \subset$ $\mathbb{F}_{p^{m}}[x] /\left\langle x^{2 \eta p^{s}}-\lambda\right\rangle$, where $0 \leq i, j \leq p^{s}$ are integers. Let $C=\left\langle\left(x^{\eta}-\xi\right)^{i}\left(x^{\eta}+\xi\right)^{j}\right\rangle \subset \mathbb{F}_{p^{m}}[x] /\left\langle x^{2 \eta p^{s}}-\lambda\right\rangle$. If $(i, j)=(0,0)$, then $C$ is the whole space $\mathbb{F}_{p^{m}}^{2 \eta p^{s}}$, and if $(i, j)=\left(p^{s}, p^{s}\right)$, then $C$ is the zero space $\{\mathbf{0}\}$. For the remaining values of $(i, j)$, the Hamming distance of $C$ is given in Table 1 .

Remark 8.23. There are some symmetries in most of the cases, so we made the following simplification in Table 1. For the cases with *, i.e., the cases except 2 and 7 , we gave the Hamming distance of $C$ when $i \geq j$. The corresponding case with $j \geq i$ has the same Hamming distance. For example in $1^{*}$, the corresponding case is $i=0$ and $0 \leq j \leq p^{s}$, and the Hamming distance is 2. Similarly in $6^{*}$, the corresponding case is $\beta p^{s-1}+1 \leq i \leq(\beta+1) p^{s-1}$ and $p^{s}-p^{s-k}+(\tau-1) p^{s-k-1}+1 \leq j \leq p^{s}-p^{s-k}+\tau p^{s-k-1}$, and the Hamming distance is $2(\beta+2)$.

The results in Table 1 still hold when the polynomials $x^{\eta}+\xi$ and $x^{\eta}-\xi$ are reducible except the fact that the cases in Table 1 do not cover all the $\lambda$-cyclic codes of length $2 \eta p^{s}$ over $\mathbb{F}_{p^{m}}$.

Remark 8.24. Note that $\left\langle\left(x^{\eta}-\xi\right)^{i}\left(x^{\eta}+\xi\right)^{j}\right\rangle, 0 \leq i, j \leq p^{s}$ are ideals of $\mathcal{R}$ independent of the fact that $x^{\eta}-\xi$ and $x^{\eta}-\xi$ are irreducible over $\mathbb{F}_{p^{m}}$. So the above results from Lemma 8.4 till Corollary 8.21 hold 
TABLE 1. The Hamming distance of all non-trivial constacyclic codes, of the form $\left\langle\left(x^{\eta}-\right.\right.$ $\left.\xi)^{i}\left(x^{\eta}+\xi\right)^{j}\right\rangle$, of length $2 \eta p^{s}$ over $\mathbb{F}_{p^{m}}$. The polynomials $x^{\eta}-\xi$ and $x^{\eta}+\xi$ are assumed to be irreducible. The parameters $1 \leq \beta^{\prime} \leq \beta \leq p-2,1 \leq \tau^{(2)}<\tau^{(1)} \leq p-1$, $1 \leq \tau, \tau^{(3)}, \tau^{(4)} \leq p-1,1 \leq k \leq s-1,1 \leq k^{\prime \prime}<k^{\prime} \leq s-1$ below are integers. For the cases with ${ }^{*}$, i.e., the cases except 2 and 7 , see Remark 8.23

\begin{tabular}{|c|c|c|c|}
\hline Case & i & $\mathbf{j}$ & $\mathrm{d}_{\mathrm{H}}(\mathrm{C})$ \\
\hline $1^{*}$ & $0<i \leq p^{s}$ & $j=0$ & 2 \\
\hline 2 & $0 \leq i \leq p^{s-1}$ & $0 \leq j \leq p^{s-1}$ & 2 \\
\hline $3^{*}$ & $p^{s-1}<i \leq 2 p^{s-1}$ & $0<j \leq p^{s-1}$ & 3 \\
\hline $4^{*}$ & $2 p^{s-1}<i \leq p^{s}$ & $0<j \leq p^{s-1}$ & 4 \\
\hline $5^{*}$ & $\beta p^{s-1}+1 \leq i \leq(\beta+1) p^{s-1}$ & $\beta^{\prime} p^{s-1}+1 \leq j \leq\left(\beta^{\prime}+1\right) p^{s-1}$ & $\begin{array}{l}\min \{\beta+2, \\
\left.2\left(\beta^{\prime}+2\right)\right\}\end{array}$ \\
\hline $6^{*}$ & $\begin{array}{l}p^{s}-p^{s-k}+(\tau-1) p^{s-k-1} \\
+1 \leq i \leq p^{s}-p^{s-k}+\tau p^{s-k-1}\end{array}$ & $\beta p^{s-1}+1 \leq j \leq(\beta+1) p^{s-1}$ & $2(\beta+2)$ \\
\hline 7 & $\begin{array}{l}p^{s}-p^{s-k}+(\tau-1) p^{s-k-1} \\
+1 \leq i \leq p^{s}-p^{s-k}+\tau p^{s-k-1}\end{array}$ & $\begin{array}{l}p^{s}-p^{s-k}+(\tau-1) p^{s-k-1} \\
+1 \leq j \leq p^{s}-p^{s-k}+\tau p^{s-k-1}\end{array}$ & $(\tau+1) p^{k}$ \\
\hline $8^{*}$ & $\begin{aligned} p^{s}-p^{s-k}+ & \left(\tau^{(1)}-1\right) p^{s-k-1} \\
+1 \leq i \leq & p^{s}-p^{s-k} \\
& +\tau^{(1)} p^{s-k-1}\end{aligned}$ & $\begin{aligned} p^{s}-p^{s-k}+ & \left(\tau^{(2)}-1\right) p^{s-k-1} \\
+1 \leq j \leq & p^{s}-p^{s-k} \\
& +\tau^{(2)} p^{s-k-1}\end{aligned}$ & $\begin{array}{l}\min \{ \\
2\left(\tau^{(2)}+1\right) p^{k}, \\
\left.\left(\tau^{(1)}+1\right) p^{k}\right\}\end{array}$ \\
\hline $9^{*}$ & $\begin{aligned} p^{s}-p^{s-k^{\prime}}+ & \left(\tau^{(3)}-1\right) p^{s-k^{\prime}-1} \\
+1 \leq i \leq & p^{s}-p^{s-k^{\prime}} \\
& +\tau^{(3)} p^{s-k^{\prime}-1}\end{aligned}$ & $\begin{aligned} p^{s}-p^{s-k^{\prime \prime}}+ & \left(\tau^{(4)}-1\right) p^{s-k^{\prime \prime}-1} \\
+1 \leq j \leq & p^{s}-p^{s-k^{\prime \prime}} \\
& +\tau^{(4)} p^{s-k^{\prime \prime}-1}\end{aligned}$ & $2\left(\tau^{(4)}+1\right) p^{k^{\prime \prime}}$ \\
\hline $10^{*}$ & $i=p^{s}$ & $\beta p^{s-1}+1 \leq j \leq(\beta+1) p^{s-1}$ & $2(\beta+2)$ \\
\hline $11^{*}$ & $i=p^{s}$ & $\begin{array}{c}p^{s}-p^{s-k}+(\tau-1) p^{s-k-1} \\
+1 \leq j \leq p^{s}-p^{s-k} \\
+\tau p^{s-k-1}\end{array}$ & $2(\tau+1) p^{k}$ \\
\hline
\end{tabular}

even when the polynomials $x^{\eta}-\xi$ and $x^{\eta}+\xi$ are reducible. But in this case, there are more $\lambda$-cyclic codes than the ones of the form $\left\langle\left(x^{\eta}-\xi\right)^{i}\left(x^{\eta}+\xi\right)^{j}\right\rangle, 0 \leq i, j \leq p^{s}$ and their Hamming distance is not given in this paper.

In the last part of this section, we determine the Hamming distance of some polycyclic codes of length $2 \eta p^{s}$ over $G R\left(p^{a}, m\right)$ whose canonical images are as above. In particular, this gives us the Hamming distance of certain constacyclic codes of length $2 \eta p^{s}$ over $G R\left(p^{a}, m\right)$. Let $\lambda_{0}, \xi_{0} \in G R\left(p^{a}, m\right)$ be units and $\bar{\lambda}_{0}=\lambda, \bar{\xi}_{0}=\xi$. So $\xi_{0}^{2 p^{s}}=\lambda_{0}$ and, $x^{\eta}-\bar{\xi}_{0}$ and $x^{\eta}+\bar{\xi}_{0}$ are irreducible. The polynomial $x^{2 \eta p^{s}}-\lambda_{0}$ factors into two coprime polynomials as

$$
x^{2 \eta p^{s}}-\lambda_{0}=x^{2 \eta p^{s}}-\xi_{0}^{2 p^{s}}=\left(x^{\eta p^{s}}-\xi_{0}^{p^{s}}\right)\left(x^{\eta p^{s}}+\xi_{0}^{p^{s}}\right) .
$$

Let $f_{1}(x)=\left(x^{\eta}-\xi_{0}\right)^{p^{s}}+p \beta_{1}(x)$ and $f_{2}(x)=\left(x^{\eta}-\xi_{0}\right)^{p^{s}}+p \beta_{2}(x)$ with $\operatorname{deg}\left(\beta_{1}(x)\right), \operatorname{deg}\left(\beta_{2}(x)\right)<\eta p^{s}$. Let $f(x)=f_{1}(x) f_{2}(x)$ and $\mathcal{R}_{0}=\frac{G R\left(p^{a}, m\right)[x]}{\langle f(x)\rangle}$. Note that $f_{1}(x)$ and $f_{2}(x)$ are primary regular polynomials and therefore we can use the arguments of Section 5 . 
By Proposition 5.1, we get $\mathcal{R}_{0}=\left\langle f_{1}(x)\right\rangle \oplus\left\langle f_{2}(x)\right\rangle$. Additionally, by Proposition 5.1, we know that $\left\langle f_{1}(x)\right\rangle \cong \frac{G R\left(p^{a}, m\right)[x]}{\left\langle f_{2}(x)\right\rangle}$ and $\left\langle f_{2}(x)\right\rangle \cong \frac{G R\left(p^{a}, m\right)[x]}{\left\langle f_{1}(x)\right\rangle}$ are local rings and the maximal ideals of $\mathcal{R}_{0}$ are $\left\langle p, x^{\eta}+\xi_{0}\right\rangle$ and $\left\langle p, x^{\eta}-\xi_{0}\right\rangle$.

Now given $g(x) \in \mathcal{R}_{0}$, we will see how to determine $\overline{\langle g(x)\rangle} \subset \mathcal{R}$. Since $\overline{\langle g(x)\rangle}=\overline{\left\langle\left(x^{\eta}-\xi\right)^{j_{0}}\left(x^{\eta}+\xi\right)^{j_{1}}\right\rangle}$, we have $\bar{g}(x)=\left(x^{\eta}-\bar{\xi}\right)^{j_{0}}\left(x^{\eta}+\bar{\xi}\right)^{j_{1}} u(x)$ where $u(x)$ is a unit in $\mathcal{R}$. In order to determine $j_{0}$, we consider the substitution $x^{i}=\left(x^{\eta}-\xi_{0}+\xi_{0}\right)^{d_{i}} x^{\ell_{i}}$ for every $i \geq \eta$, we get

$$
\begin{aligned}
g(x) & =a_{L} x^{L}+\cdots+a_{\eta} x^{\eta}+a_{\eta-1} x^{\eta-1}+\cdots+a_{0} \\
& =\left(x^{\eta}-\xi_{0}\right)^{d_{L}} h_{d_{L}}(x)+\left(x^{\eta}-\xi_{0}\right)^{d_{L}-1} h_{d_{L}-1}(x)+\cdots+h_{0}(x)
\end{aligned}
$$

where $h_{i}(x)$ are polynomials such that $\operatorname{deg}\left(h_{i}(x)\right)<\eta$ for $d_{L} \geq i \geq 0$. Then $j_{0}$ is the least integer with the property $p \nmid h_{j_{0}}(x)$. Similarly, via the substitution $x^{i}=\left(x^{\eta}+\xi_{0}-\xi_{0}\right)^{d_{i}} x^{\ell_{i}}$ for every $i \geq \eta$, the integer $j_{1}$ can be determined.

Let $C=\left\langle g_{1}(x), \ldots, g_{r}(x)\right\rangle \triangleleft \mathcal{R}_{0}$ be a polycyclic code, where the generators are as in Theorem 5.5. By Theorem [5.6, we have $d_{H}(C)=d_{H}\left(\overline{\left\langle g_{r}(x)\right\rangle}\right)$. The canonical image $\overline{\left\langle g_{r}(x)\right\rangle}$ of $\left\langle g_{r}(x)\right\rangle$ can be determined as described above. Say $\overline{\left\langle g_{r}(x)\right\rangle}=\overline{\left\langle\left(x^{\eta}-\xi\right)^{\hat{i}}\left(x^{\eta}+\xi\right)^{\hat{j}}\right\rangle}$ for some $0 \leq \hat{i}, \hat{j} \leq p^{s}$. Then $d_{H}\left(\overline{\left\langle\left(x^{\eta}-\xi\right)^{\hat{i}}\left(x^{\eta}+\xi\right)^{\hat{j}}\right\rangle}\right)$ can be determined using Theorem 8.22 .

Remark 8.25. Note that $x^{\eta p^{s}}-\xi_{0}^{p^{s}}=\left(x^{\eta}-\xi_{0}\right)^{p^{s}}+p \hat{\beta}_{1}(x)$ and $x^{\eta p^{s}}+\xi_{0}^{p^{s}}=\left(x^{\eta}+\xi_{0}\right)^{p^{s}}+p \hat{\beta}_{2}(x)$ for some $\hat{\beta}_{1}(x), \hat{\beta}_{2}(x) \in \mathcal{R}_{0}$. In the above setup, if we take $f_{1}(x)=\left(x^{\eta}-\xi_{0}\right)^{p^{s}}+p \hat{\beta}_{1}(x)$ and $f_{2}(x)=$ $\left(x^{\eta}+\xi_{0}\right)^{p^{s}}+p \hat{\beta}_{2}(x)$, then we obtain the Hamming distance of $\lambda$-cyclic codes of length $2 \eta p^{s}$ over $G R\left(p^{a}, m\right)$.

\section{ACKNOWLEDGMENTS}

Ferruh Özbudak is partially supported by TÜBİTAK under Grant No. TBAG-109T672.

\section{REFERENCES}

[1] Taher Abualrub and Robert Oehmke. On the generators of $\mathbb{Z}_{4}$ cyclic codes of length $2^{e}$. IEEE Trans. Inform. Theory, 49(9):2126-2133, 2003.

[2] Elwyn R. Berlekamp. Algebraic coding theory. McGraw-Hill Book Co., New York, 1968.

[3] Gilberto Bini and Flaminio Flamini. Finite commutative rings and their applications. The Kluwer International Series in Engineering and Computer Science, 680. Kluwer Academic Publishers, Boston, MA, 2002.

[4] Jason Thomas Blackford. Negacyclic codes over $Z_{4}$ of even length. IEEE Trasns. Inform. Theory, 49(6):1417-1424, 2003.

[5] Jason Thomas Blackford and Dwijendra K. Ray-Chaudhuri. A transform approach to permutation groups of cyclic codes over Galois rings. IEEE Trans. Inform. Theory, 46(7):2350-2358, 2000.

[6] A. R. Calderbank and N. J. A. Sloane. Modular and p-adic cyclic codes. Des. Codes Cryptogr., 6(1):21-35, 1995.

[7] Guy Castagnoli, James L. Massey, Philipp A. Schoeller, and Niklaus von Seemann. On repeated-root cyclic codes. IEEE Trans. Inform. Theory, 37(2):337-342, 1991.

[8] Hai Q. Dinh. Negacyclic codes of length $2^{s}$ over Galois rings. IEEE Trans. Inform. Theory, 51(12):4252-4262, 2005.

[9] Hai Q. Dinh. Complete distances of all negacyclic codes of length $2^{\text {s }}$ over $\mathbb{Z}_{2^{a}}$. IEEE Trans. Inform. Theory, 53(1):147$161,2007$.

[10] Hai Q. Dinh. On the linear ordering of some classes of negacyclic and cyclic codes and their distance distributions. Finite Fields Appl., 14(1):22-40, 2008.

[11] Hai Q. Dinh. Constacyclic codes of length $2^{s}$ over Galois extension rings of $\mathbb{F}_{2}+u \mathbb{F}_{2}$. IEEE Trans. Inform. Theory, 55(4):1730-1740, 2009.

[12] Hai Q. Dinh. Constacyclic codes of length $p^{s}$ over $\mathbb{F}_{p^{m}}+u \mathbb{F}_{p^{m}}$. J. Algebra, 324(5):940-950, 2010. 
[13] Hai Q. Dinh, Sergio R. López-Permouth, and Steve Szabo. On the structure of cyclic and negacyclic codes over finite chain rings. In Patrick Solé, editor, Codes Over Rings, volume 6 of Series on Coding Theory and Cryptology, pages 22-59. World Scientific Publ Co Pte Ltd, 2009.

[14] Hai Quang Dinh and Sergio R. López-Permouth. Cyclic and negacyclic codes over finite chain rings. IEEE Trans. Inform. Theory, 50(8):1728-1744, 2004.

[15] Steven T. Dougherty and Young Ho Park. On modular cyclic codes. Finite Fields Appl., 13(1):31-57, 2007.

[16] Andrew Granville. Arithmetic properties of binomial coefficients. I. Binomial coefficients modulo prime powers. In Organic mathematics (Burnaby, BC, 1995), volume 20 of CMS Conf. Proc., pages 253-276. Amer. Math. Soc., Providence, RI, 1997.

[17] A. Roger Hammons, Jr., P. Vijay Kumar, A. R. Calderbank, N. J. A. Sloane, and Patrick Solé. The $\mathbf{Z}_{4}$-linearity of Kerdock, Preparata, Goethals, and related codes. IEEE Trans. Inform. Theory, 40(2):301-319, 1994.

[18] Xiaoshan Kai and Shixin Zhu. On the distance of cyclic codes of length $2^{e}$ over $\mathbb{Z}_{4}$. Discrete Math., 310(1):12-20, 2010.

[19] Pramod Kanwar and Sergio R. López-Permouth. Cyclic codes over the integers modulo $p^{m}$. Finite Fields Appl., 3(4):334352, 1997.

[20] Han Mao Kiah, Ka Hin Leung, and San Ling. Cyclic codes over $\operatorname{GR}\left(p^{2}, m\right)$ of length $p^{k}$. Finite Fields Appl., 14(3):834846, 2008.

[21] P. Vijay Kumar, Tor Helleseth, A. R. Calderbank, and A. Roger Hammons, Jr. Large families of quaternary sequences with low correlation. IEEE Trans. Inform. Theory, 42(2):579-592, 1996.

[22] Sergio R. López-Permouth and Steve Szabo. On the Hamming weight of repeated root cyclic and negacyclic codes over Galois rings. Adv. Math. Commun., 3(4):409-420, 2009.

[23] James L. Massey, Daniel J. Costello, and Jørn Justesen. Polynomial weights and code constructions. IEEE Trans. Information Theory, IT-19:101-110, 1973.

[24] Bernard R. McDonald. Finite rings with identity. Marcel Dekker Inc., New York, 1974. Pure and Applied Mathematics, Vol. 28.

[25] G. H. Norton and A. Sălăgean. Cyclic codes and minimal strong Gröbner bases over a principal ideal ring. Finite Fields Appl., 9(2):237-249, 2003.

[26] Graham H. Norton and Ana Sălăgean. On the Hamming distance of linear codes over a finite chain ring. IEEE Trans. Inform. Theory, 46(3):1060-1067, 2000.

[27] Graham H. Norton and Ana Sălăgean. On the structure of linear and cyclic codes over a finite chain ring. Appl. Algebra Engrg. Comm. Comput., 10(6):489-506, 2000.

[28] Graham H. Norton and Ana Sălăgean. Strong Gröbner bases for polynomials over a principal ideal ring. Bull. Austral. Math. Soc., 64(3):505-528, 2001.

[29] Hakan Özadam and Ferruh Özbudak. A note on negacyclic and cyclic codes of length $p^{s}$ over a finite field of characteristic p. Adv. Math. Commun., 3(3):265-271, 2009.

[30] Vera S. Pless and Zhongqiang Qian. Cyclic codes and quadratic residue codes over $Z_{4}$. IEEE Trans. Inform. Theory, 42(5):1594-1600, 1996.

[31] Ron M. Roth and Gadiel Seroussi. On cyclic MDS codes of length q over GF(q). IEEE Trans. Inform. Theory, 32(2):284285, 1986.

[32] Ana Sălăgean. Repeated-root cyclic and negacyclic codes over a finite chain ring. Discrete Appl. Math., 154(2):413-419, 2006.

[33] Li-zhong Tang, Cheong Boon Soh, and Erry Gunawan. A note on the $q$-ary image of a $q^{m}$-ary repeated-root cyclic code. IEEE Trans. Inform. Theory, 43(2):732-737, 1997.

[34] J. H. van Lint. Repeated-root cyclic codes. IEEE Trans. Inform. Theory, 37(2):343-345, 1991.

[35] Gerardo Vega and Jacques Wolfmann. Some families of $\mathbb{Z}_{4}$-cyclic codes. Finite Fields Appl., 10(4):530-539, 2004.

[36] Jacques Wolfmann. Negacyclic and cyclic codes over $Z_{4}$. IEEE Trans. Inform. Theory, 45(7):2527-2532, 1999.

[37] Jacques Wolfmann. Binary images of cyclic codes over $\mathbb{Z}_{4}$. IEEE Trans. Inform. Theory, 47(5):1773-1779, 2001.

[38] Karl-Heinz Zimmermann. On generalizations of repeated-root cyclic codes. IEEE Trans. Inform. Theory, 42(2):641-649, 1996. 